Épijournal de Géométrie Algébrique
epiga.episciences.org
Volume 3 (2019), Article Nr. 13

\title{
Weighted projective lines and rational surface singularities
}

\author{
Osamu Iyama and Michael Wemyss
}

\begin{abstract}
In this paper we study rational surface singularities $R$ with star shaped dual graphs, and under very mild assumptions on the self-intersection numbers we give an explicit description of all their special Cohen-Macaulay modules. We do this by realising $R$ as a certain $\mathbb{Z}$-graded Veronese subring $S^{\vec{x}}$ of the homogeneous coordinate ring $S$ of the Geigle-Lenzing weighted projective line $\mathbb{X}$, and we realise the special $\mathrm{CM}$ modules as explicitly described summands of the canonical tilting bundle on $\mathrm{X}$. We then give a second proof that these are special CM modules by comparing qgr $S^{\vec{x}}$ and coh $\mathbb{X}$, and we also give a necessary and sufficient combinatorial criterion for these to be equivalent categories. In turn, we show that $\operatorname{qgr} S^{\vec{x}}$ is equivalent to $\operatorname{qgr} \Gamma$ where $\Gamma$ is the corresponding reconstruction algebra, and that the degree zero piece of $\Gamma$ coincides with Ringel's canonical algebra. This implies that $\Gamma$ contains the canonical algebra and furthermore qgr $\Gamma$ is derived equivalent to the canonical algebra, thus linking the reconstruction algebra of rational surface singularities to the canonical algebra of representation theory.
\end{abstract}

Keywords. Weighted projective line, rational surface singularities, reconstruction algebra, canonical algebra, tilting theory, Cohen-Macaulay modules

2010 Mathematics Subject Classification. 32S25 (primary); 14E16, 16G50, 18E30 (secondary)

[Français]

Titre. Droites projectives à poids et singularités rationnelles de surfaces

Résumé. Dans cet article, nous étudions les singularités rationnelles de surfaces $R$ de graphes duaux étoilés. Sous de légères hypothèses sur les nombres d'auto-intersection, nous donnons une description explicite de tous leurs modules Cohen-Macaulay spéciaux. Pour cela, nous réalisons $R$ comme un certain sous-anneau de Veronese $\mathbb{Z}$-gradué $S^{\vec{x}}$ de l'anneau de coordonnées homogènes $S$ de la droite projective pondérée de Geigle-Lenzing $\mathbb{X}$ et nous réalisons les modules $\mathrm{CM}$ spéciaux comme des facteurs directs explicites du fibré basculant canonique de $\mathbb{X}$. Nous donnons ensuite une seconde démonstration du fait que ce sont des modules CM spéciaux en comparant qgr $S^{\vec{x}}$ et coh $X$, et nous énonçons également un critère combinatoire nécessaire et suffisant d'équivalence pour ces deux catégories. En outre nous montrons que qgr $S^{\vec{x}}$ est équivalente à qgr $\Gamma$ où $\Gamma$ est l'algèbre de reconstruction correspondante, et que la partie de degré zéro de $\Gamma$ coïncide avec l'algèbre canonique de Ringel. Cela implique que $\Gamma$ contient l'algèbre canonique et de plus que qgr $\Gamma$ est équivalente au sens dérivé à l'algèbre canonique, reliant ainsi l'algèbre de reconstruction des singularités rationnelles de surfaces à la théorie des représentations de l'algèbre canonique.

Received by the Editors on August 18, 2018. Accepted on August 7, 2019.

Osamu Iyama

Graduate School of Mathematics, Nagoya University, Chikusa-ku, Nagoya, 464-8602, Japan

e-mail: iyama@math.nagoya-u.ac.jp

Michael Wemyss

School of Mathematics and Statistics, University of Glasgow, University Place, Glasgow, G12 8QQ, UK

e-mail: michael.wemyss@glasgow.ac.uk

Osamu Iyama was supported by JSPS Grant-in-Aid for Scientific Research (B)24340004, (B)16H03923, (C)23540045 and (S)15H05738, and Michael Wemyss was supported by EPSRC grant EP/K021400/2. Both were partially supported by the American Institute of Mathematics. 


\section{Contents}

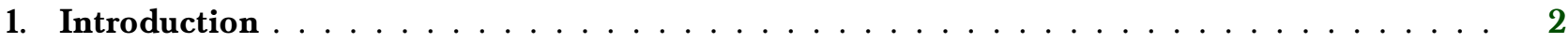

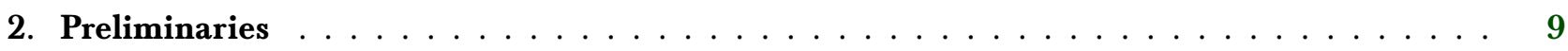

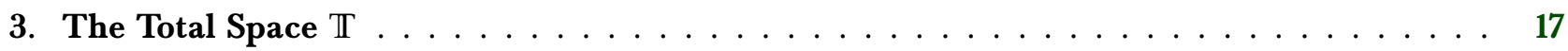

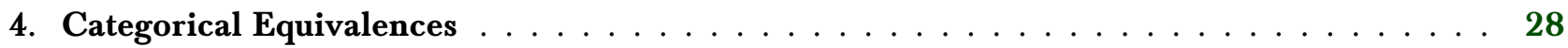

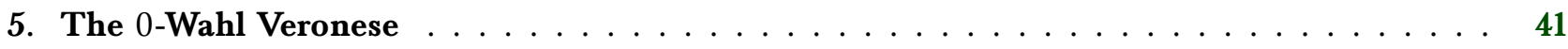

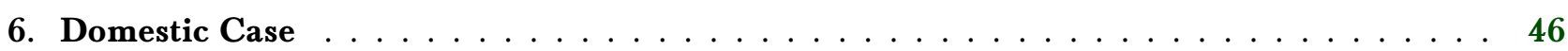

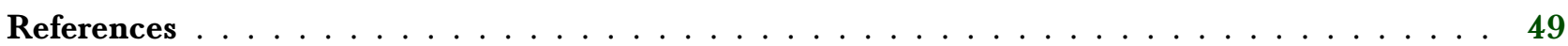

\section{Introduction}

\subsection{Motivation and Overview}

It is well known that any rational surface singularity has only finitely many indecomposable special CM modules, but it is in general a difficult task to classify and describe them explicitly. In this paper we use the combinatorial structure encoded in the homogeneous coordinate ring $S$ of the Geigle-Lenzing weighted projective line $\mathbb{X}$ to solve this problem for a large class of examples arising from star shaped dual graphs, extending our previous work [IW] to cover a much larger class of varieties. In the process, we link $S$, its Veronese subrings, the reconstruction algebra and the canonical algebra, through a range of categorical equivalences.

A hint of a connection between rational surface singularities and the canonical algebra can be found in the lecture notes [R2]. In his study of the canonical algebra $\Lambda_{\mathrm{p}, \lambda}$, Ringel drew pictures [R2, p196] of canonical tilting bundles on $\mathbb{X}$ for the cases $\mathbf{p}=(2,3,3),(2,3,4)$ and $(2,3,5)$, which correspond to Dynkin diagrams $E_{6}, E_{7}$ and $E_{8}$. For example, in the $E_{7}$ case, Ringel's picture is the following.

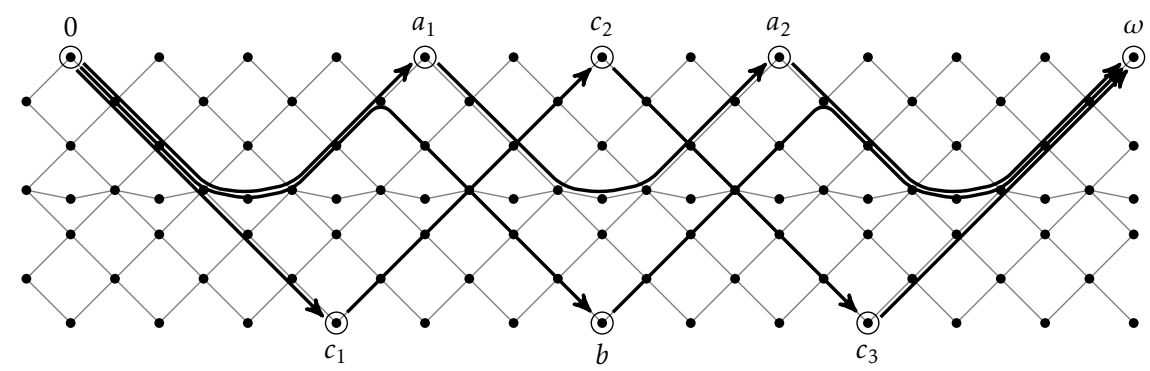

What is remarkable is that all of Ringel's pictures are identical to ones the authors drew in [IW, \$7-9] when classifying special CM modules for certain families of quotient singularities $\mathbb{k}[[x, y]]^{G}$ with $G \leq \mathrm{GL}(2, \mathbb{k})$. For example, [IW, 8.2] contains the following picture, indicating the positions of special CM modules in the AR quiver of $\mathbb{k}[[x, y]]^{\mathbb{O}_{13}}$.

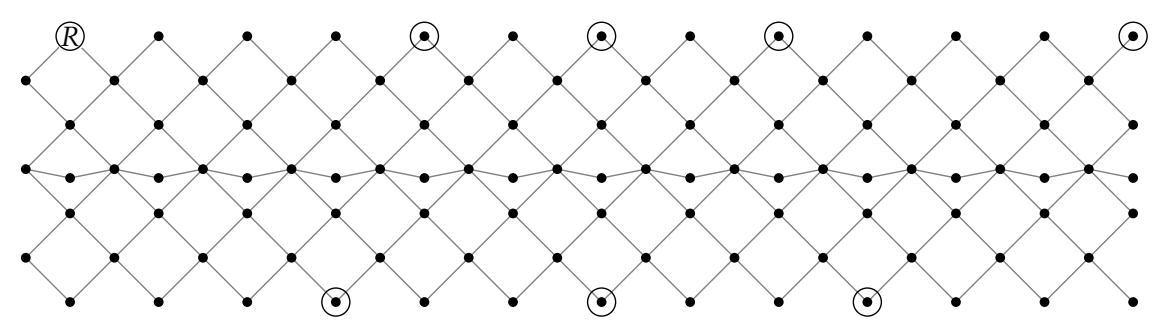


Further, although they were not drawn in [IW], the arrows in (1.A) are implicit in the calculation of the quiver of the corresponding reconstruction algebra [W2, §4]. This paper grew out of trying to give a conceptual explanation for this coincidence, since a connection between the mathematics underpinning the two pictures did not seem to be known.

In fact, the connection turns out to be explained by a very general phenomenon. Recall first that one of the basic properties of the canonical algebra $\Lambda_{\mathbf{p}, \lambda}$ is that there is always a derived equivalence [GL1]

$$
\mathrm{D}^{\mathrm{b}}\left(\operatorname{coh} \mathrm{X}_{\mathbf{p}, \lambda}\right) \simeq \mathrm{D}^{\mathrm{b}}\left(\bmod \Lambda_{\mathbf{p}, \lambda}\right)
$$

where $\operatorname{coh} \mathbb{X}_{p, \lambda}$ is the weighted projective line of Geigle-Lenzing (for details, see §1.2). Thus, to explain the above coincidence, we are led to consider the possibility of linking the weighted projective line, viewed as a Deligne-Mumford stack, to the study of rational surface singularities. However, the weighted projective line $X_{p, \lambda}$ cannot itself be the stack that we are after, since it only has dimension one, and rational surface singularities have, by definition, dimension two.

We need to increase the dimension, and the most naive way to do this is to consider the total space of a line bundle over $\mathbb{X}_{\mathrm{p}, \lambda}$. We thus choose any member of the grading group $\vec{x} \in \mathbb{L}$ and consider the total space stack $\operatorname{Tot}\left(\mathcal{O}_{\mathbf{X}}(-\vec{x})\right)$ (for definition, see $\left.\S 1.2\right)$. From tilting on this and its coarse moduli, under mild assumptions we prove that the Veronese subring $S^{\vec{x}}:=\bigoplus_{i \in \mathbb{Z}} S_{i \vec{x}}$ is a weighted homogeneous rational surface singularity, giving the first concrete connection between the above two settings. Furthermore, from the stack $\operatorname{Tot}\left(\mathcal{O}_{\mathbb{X}}(-\vec{x})\right)$ we then describe the special CM $S^{\vec{x}}$-modules, and give precise information regarding the minimal resolution of Spec $S^{\vec{x}}$ and its derived category.

Our results recover known special cases, such as the domestic case (corresponding to Dynkin diagrams), where it is known that $S^{-\vec{\omega}}$ is a simple singularity. In that setting there is an equivalence $\mathrm{CM}^{\mathbb{L}} S \simeq \mathrm{CM}^{\mathbb{Z}} S^{-\vec{\omega}}$, and this leads us to investigate more general categorical equivalences. We do this for very general $S$ and $\vec{x}$, and through a range of categorical equivalences we are then able to relate $\mathrm{CM}^{\mathbb{Z}} S^{\vec{x}}$ and vect $\mathbb{X}$, which finally allows us in Section 6 to explain categorically why the above two pictures must be the same.

We now describe our results in detail.

\subsection{Veronese Subrings and Special CM modules}

Throughout, let $\mathbb{k}$ denote an algebraically closed field of characteristic zero. For any $n \geq 0$, choose positive integers $p_{1}, \ldots, p_{n}$ with all $p_{i} \geq 2$ and set $\mathbf{p}:=\left(p_{1}, \ldots, p_{n}\right)$. Furthermore, choose pairwise distinct points $\lambda_{1}, \ldots, \lambda_{n}$ in $\mathbb{P}^{1}$, and denote $\lambda:=\left(\lambda_{1}, \ldots, \lambda_{n}\right)$. Let $\ell_{i}\left(t_{0}, t_{1}\right) \in \mathbb{k}\left[t_{0}, t_{1}\right]$ be the linear form defining $\lambda_{i}$, and write

$$
S_{\mathbf{p}, \lambda}=S:=\frac{\mathbb{k}\left[t_{0}, t_{1}, x_{1}, \ldots, x_{n}\right]}{\left(x_{i}^{p_{i}}-\ell_{i}\left(t_{0}, t_{1}\right) \mid 1 \leq i \leq n\right)} .
$$

Moreover, let $\mathbb{L}=\mathbb{L}\left(p_{1}, \ldots, p_{n}\right)$ be the abelian group generated by the elements $\vec{x}_{1}, \ldots, \vec{x}_{n}$ subject to the relations $p_{1} \vec{x}_{1}=p_{2} \vec{x}_{2}=\cdots=p_{n} \vec{x}_{n}=: \vec{c}$. With this input $S$ is an $\mathbb{L}$-graded algebra with $\operatorname{deg} x_{i}:=\vec{x}_{i}$ and $\operatorname{deg} t_{j}:=\vec{c}$, and $\mathbb{L}$ is a rank one abelian group, possibly containing torsion. Often we normalize $\lambda$ so that $\lambda_{1}=0, \lambda_{2}=\infty$ and $\lambda_{3}=1$, however it is important for changing parameters later that we allow ourselves flexibility.

From this, consider the stack

$$
\mathbb{X}_{\mathbf{p}, \lambda}=\mathbb{X}:=\left[\left(\operatorname{Spec} S_{\mathbf{p}, \lambda} \backslash 0\right) / \operatorname{Spec} \mathbb{k} \mathbb{L}\right],
$$

with coarse moduli space denoted $X_{\mathbf{p}, \lambda}=X$. It is well known that $X \cong \mathbb{P}^{1}$, regardless of $\mathbf{p}$ and $\lambda$ (see 2.1(2)).

To increase the dimension we choose an element $\vec{x} \in \mathbb{L}$ and consider both the Veronese subring given by $S^{\vec{x}}:=\bigoplus_{i \in \mathbb{Z}} S_{i \vec{x}}$ and the total space stack

$$
\mathbb{T}^{\vec{x}}=\operatorname{Tot}\left(\mathcal{O}_{\mathbf{X}_{\mathbf{p}, \lambda}}(-\vec{x})\right):=\left[\left(\operatorname{Spec} S_{\mathbf{p}, \lambda} \backslash 0 \times \operatorname{Spec} \mathbb{k}[t]\right) / \operatorname{Spec} \mathbb{k} \mathbb{L}\right],
$$


where $\mathbb{L}$ acts on $t$ with weight $-\vec{x}$. Writing $\vec{x}=\sum_{i=1}^{n} a_{i} \vec{x}_{i}+a \vec{c}$ in normal form (see 2.1(1)), we show in 3.2 that the coarse moduli space $T^{\vec{x}}$ is a surface containing a $\mathbb{P}^{1}$, and on that $\mathbb{P}^{1}$ complete locally the singularities of $T^{\vec{x}}$ are of the form

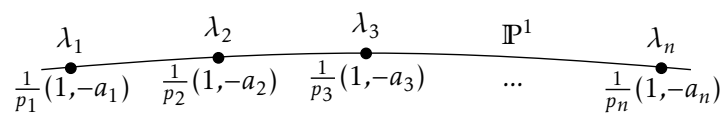

where for notation see 2.13 .

As is standard, positivity conditions on $\vec{x}$ are needed in order to contract the zero section of $T^{\vec{x}}$. It turns out that the correct notion is to assume that $\vec{x} \in \mathbb{L}$ is not torsion, and further that $\vec{\omega}-i \vec{x} \notin \mathbb{L}_{+}$for all $i \geq 0$ (for the definition of $\vec{\omega}$ and $\mathbb{L}_{+}$see 2.1(1)). Under this mild positivity restriction, we show in 3.7 that there is a canonical morphism

$$
\gamma: T^{\vec{x}} \rightarrow \operatorname{Spec} S^{\vec{x}}
$$

satisfying $\mathbf{R} \gamma_{*} \mathcal{O}_{T^{\vec{x}}}=\mathcal{O}_{S^{\vec{x}}}$, and further in 3.10 that $\gamma$ is projective birational. Composing $\gamma$ with the minimal resolution $\varphi: Y^{\vec{x}} \rightarrow T^{\vec{x}}$ of $T^{\vec{x}}$, gives the following.

Theorem 1.1 (=3.11). If $\vec{x} \in \mathbb{L}$ is not torsion, and $\vec{\omega}-i \vec{x} \notin \mathbb{L}_{+}$for all $i \geq 0$, then $S^{\vec{x}}$ is a rational surface singularity.

In the setting of the above theorem, all the datum can be summarized by the following commutative diagram

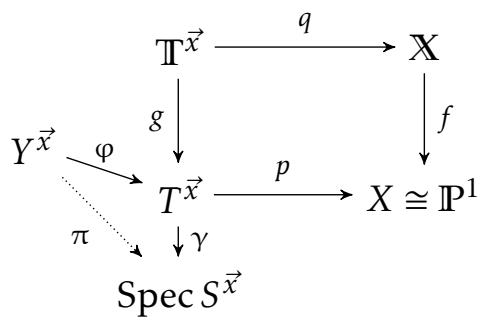

We remark that the coarse moduli space $T^{\vec{x}}$ is a singular line bundle in the sense of Dolgachev [D, §4] and Pinkham [P, §3], which also appears in the work of Orlik-Wagreich [OW] and many others. However, the key difference in our approach is that the grading group giving the quotient is $\mathbb{L}$ not $\mathbb{Z}$, and indeed it is the extra combinatorial structure of $\mathbb{L}$ that allows us to extract the geometry much more easily.

It is in fact easy to check (see 3.6(1)) that the positivity condition on $\vec{x}$ in 1.1 is satisfied if $0 \neq \vec{x} \in \mathbb{L}_{+}$. This setting is particularly pleasant, since tilting behaves well.

Theorem $1.2(=3.14)$. If $0 \neq \vec{x} \in \mathbb{L}_{+}$, then $p^{*}\left(\mathcal{O}_{\mathbb{P}^{1}} \oplus \mathcal{O}_{\mathbb{P}^{1}}(1)\right)$ is a tilting bundle on $T^{\vec{x}}$.

Writing $\mathcal{E}:=\bigoplus_{i \in[0, \vec{c}]} \mathcal{O}_{\mathbf{X}}(i)$ for the Geigle-Lenzing tilting bundle on $\mathbb{X}$ [GL1], our next main result is then the following.

Theorem 1.3. If $0 \neq \vec{x} \in \mathbb{L}_{+}$, then with notation as in (1.C),

(1) $(=3.13) q^{*} \mathcal{E}$ is a tilting bundle on $\mathbb{T}^{\vec{x}}$ such that

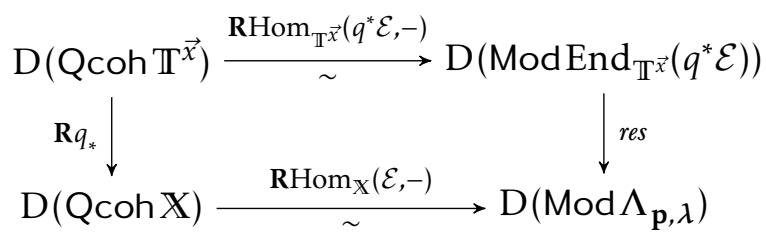

commutes, where $\Lambda_{\mathrm{p}, \lambda}$ is the canonical algebra of Ringel.

(2) (=3.21) There is a fully faithful embedding $\mathrm{D}^{\mathrm{b}}\left(\operatorname{coh} Y^{\vec{x}}\right) \hookrightarrow \mathrm{D}^{\mathrm{b}}\left(\operatorname{coh} \mathbb{T}^{\vec{x}}\right)$. 
It is by analysing $1.3(2)$ that we are able to extract the special CM $S^{\vec{x}}$-modules below. We show in $\S 4.3$ that for any non-torsion $\vec{x}=\sum_{i=1}^{n} a_{i} \vec{x}_{i}+a \vec{c}$ we can change parameters and replace $(\mathbf{p}, \lambda, \vec{x})$ by $\left(\mathbf{p}^{\prime}:=\left(\mathrm{p}_{i} \mid i \in I\right), \lambda^{\prime}, \overrightarrow{\mathrm{x}}:=\sum_{i \in I} \mathrm{a}_{i} \vec{x}_{i}+a \overrightarrow{\mathrm{c}} \in \mathbb{L}^{\prime}\right)$ such that $S_{\mathbf{p}, \lambda}^{\vec{x}}=S_{\mathbf{p}^{\prime}, \lambda^{\prime}}^{\vec{x}}$ and $\left(\mathrm{p}_{i}, \mathrm{a}_{i}\right)=1$ holds. See 4.10 for full details. With this in mind, we are then able to precisely control when the embedding in 1.3(2) is an equivalence.

Proposition 1.4 (=4.8). If $0 \neq \vec{x}=\sum_{i=1}^{n} a_{i} \vec{x}_{i}+a \vec{c} \in \mathbb{L}_{+}$with $\left(p_{i}, a_{i}\right)=1$ for all $1 \leq i \leq n$, then the embedding in 1.3(2) is an equivalence if and only if every $a_{i}$ is 1 , that is $\vec{x}=\sum_{i=1}^{n} \vec{x}_{i}+a \vec{c}$.

Combining the above tilting result with (1.B) and a combinatorial argument, we are in fact able to determine the precise dual graph (for definition see 2.5) of the morphism $\pi$ in (1.C). Recall that for each $\frac{1}{p_{i}}\left(1,-a_{i}\right)$ in $(1 . \mathrm{B})$ with $a_{i} \neq 0$, we can consider the Hirzebruch-Jung continued fraction expansion

$$
\frac{p_{i}}{p_{i}-a_{i}}=\alpha_{i 1}-\frac{1}{\alpha_{i 2}-\frac{1}{\alpha_{i 3}-\frac{1}{(\ldots)}}}:=\left[\alpha_{i 1}, \ldots, \alpha_{i m_{i}}\right],
$$

with each $\alpha_{i j} \geq 2$; see $\S 2.4$ for full details.

Theorem $1.5(=3.16,4.19)$. Let $0 \neq \vec{x} \in \mathbb{L}_{+}$and as above write $\vec{x}=\sum_{i=1}^{n} a_{i} \vec{x}_{i}+a \vec{c}$ in normal form. Then the dual graph of the morphism $\pi: Y^{\vec{x}} \rightarrow \operatorname{Spec} S^{\vec{x}}$ is

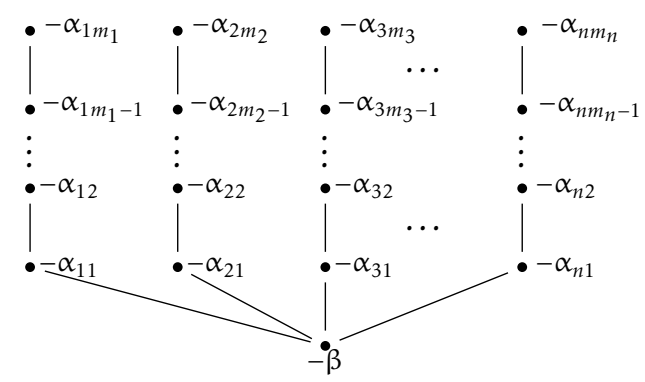

where the arm $\left[\alpha_{i 1}, \ldots, \alpha_{i m_{i}}\right]$ corresponds to $i \in\{1, \ldots, n\}$ with $a_{i} \neq 0$, and the $\alpha_{i j}$ are given by the HirzebruchJung continued fractions in (1.D). Furthermore, writing $v=\#\left\{i \mid a_{i} \neq 0\right\}$ for the number of arms, we have $\beta=a+v$.

We first establish in 3.17 that $\pi$ is the minimal resolution if and only if $\vec{x} \notin[0, \vec{c}]$. Theorem 1.5 is then proved by splitting into the two cases $\vec{x} \notin[0, \vec{c}]$ and $\vec{x} \in[0, \vec{c}]$, with the verification in both cases being rather different. Note that the case $\vec{x} \in[0, \vec{c}]$ is degenerate as $[0, \vec{c}]$ is a finite interval, containing only those $\vec{x}$ of the form $a_{i} \vec{x}_{i}$ for some $i$ and some $0 \leq a_{i} \leq p_{i}$. In this paper we are mostly interested in special CM modules and these are defined using the minimal resolution: this is why below the condition $\vec{x} \notin[0, \vec{c}]$ often appears.

We remark that for $0 \neq \vec{x} \in \mathbb{L}_{+}, S^{\vec{x}}$ is rarely a quotient singularity, and it is even more rare for it to be ADE. Nevertheless, the dual graphs of all quotient singularities $\mathbb{k}^{2} / G$ (where $G$ is a small subgroup of $\operatorname{GL}(2, \mathbb{k})$ ) are known [B3], and so whether $S^{\vec{x}}$ is a quotient singularity can, if needed, be immediately determined by 1.5 , after contracting (-1)-curves if necessary.

One key observation in this paper is that controlling the stack $\mathbb{T}^{\vec{x}}$ allows us not only to obtain a rational surface singularity $S^{\vec{x}}$, with its dual graph, but furthermore it also allows us to determine the special CM $S^{\vec{x}}$-modules. Indeed, in effect we simply compare the two resolutions

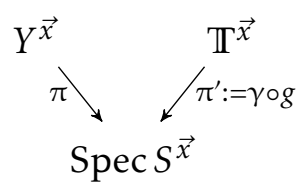


constructed above. It is known that $Y^{\vec{x}}$ has a tilting bundle $\mathcal{M}$ [VdB, W6], and by $1.3(1)$ that $\mathbb{T}^{\vec{x}}$ has tilting bundle $q^{*} \mathcal{E}$, where $\mathcal{E}$ is the Geigle-Lenzing tilting bundle on $\mathbb{X}$. Pushing these down to Spec $S^{\vec{x}}$ gives the following result. Throughout, we write $\mathrm{SCM} S^{\vec{x}}$ for the category of special CM $S^{\vec{x}}$-modules; for definitions see $\S 2.3$. For $\vec{y} \in \mathbb{L}$, write $S(\vec{y})^{\vec{x}}:=\bigoplus_{i \in \mathbb{Z}} S_{\vec{y}+i \vec{x}}$.

Theorem 1.6. If $0 \neq \vec{x} \in \mathbb{L}_{+}$with $\vec{x} \notin[0, \vec{c}]$, then the following hold.

(1) [W6] $\operatorname{SCM} S^{\vec{x}}=\operatorname{add} \pi_{*} \mathcal{M}$.

(2) $(=3.21) \pi_{*} \mathcal{M}$ is a summand of $\pi_{*}^{\prime}\left(q^{*} \mathcal{E}\right)=\bigoplus_{\vec{y} \in[0, \vec{c}]} S(\vec{y})^{\vec{x}}$.

Furthermore, we say precisely which summands of $\pi_{*}^{\prime}\left(q^{*} \mathcal{E}\right)$ give the special CM modules. As notation, recall that the $i$-series associated to the Hirzebruch-Jung continued fraction expansion $\frac{r}{a}=\left[\alpha_{1}, \ldots, \alpha_{m}\right]$ is defined as $i_{0}=r, i_{1}=a$ and $i_{t}=\alpha_{t-1} i_{t-1}-i_{t-2}$ for all $t$ with $2 \leq t \leq m+1$, and we write

$$
I(r, a):=\left\{i_{0}, i_{1}, \ldots, i_{m+1}\right\} .
$$

As convention $I(r, r)=\emptyset$.

Theorem $1.7(=3.18,4.15)$. If $\vec{x} \in \mathbb{L}_{+}$with $\vec{x} \notin[0, \vec{c}]$, write $\vec{x}$ in normal form $\vec{x}=\sum_{i=1}^{n} a_{i} \vec{x}_{i}+a \vec{c}$. Then

$$
\operatorname{SCM} S^{\vec{x}}=\operatorname{add}\left\{S\left(u \vec{x}_{j}\right)^{\vec{x}} \mid j \in[1, n], u \in I\left(p_{j}, p_{j}-a_{j}\right)\right\} .
$$

This allows us to construct both $R=S^{\vec{x}}$, and its special CM modules, for (almost) every star shaped dual graph. We remark that this is the first time that special CM modules have been classified in any example with infinite $\mathrm{CM}$ representation type, and indeed, due to the non-tautness of the dual graph, in an uncountable family of examples. For simplicity in this paper, we restrict the explicitness to certain families of examples, and refer the reader to $\$ 5.2$ for more details.

By construction, all the special CM $S^{\vec{x}}$-modules have a natural $\mathbb{Z}$-grading, and we let $N$ denote their sum. By definition the reconstruction algebra is defined to be $\Gamma_{\vec{x}}:=\operatorname{End}_{\vec{S} \vec{x}}(N)$, and in this setting it inherits a $\mathbb{N}$-grading from the grading of the special CM modules in 1.7. In general, it is not generated in degree one over its degree zero piece, but nevertheless the degree zero piece is always some canonical algebra of Ringel. We state the first half of the following result vaguely, giving a much more precise description of the parameters in 4.21 .

Proposition 1.8. Suppose that $x \in \mathbb{L}_{+}$with $\vec{x} \notin[0, \vec{c}]$.

(1) $(=4.21)$ The degree zero part of $\Gamma_{\vec{x}}$ is isomorphic to the canonical algebra $\Lambda_{\mathbf{q}, \mu}$, for some suitable parameters $(\mathbf{q}, \mu)$.

(2) (=5.8) For $\vec{s}:=\sum_{i=1}^{n} \vec{x}_{i}$, then $\Gamma_{\vec{s}}$ is generated in degree one over its degree zero piece. Moreover the degree zero piece is the canonical algebra $\Lambda_{\mathbf{p}, \lambda}$.

\subsection{Geigle-Lenzing Weighted Projective Lines via Rational Surface Singularities}

For an abelian group $G$ and a $G$-graded noetherian $\mathbb{k}$-algebra, we write $\bmod ^{G} A$ for the category of finitely generated $G$-graded $A$-modules, $\bmod _{0}^{G} A$ for the subcategory of finite dimensional modules, and $\mathrm{qgr}^{G} A:=\bmod ^{G} A / \bmod _{0}^{G} A$ for the Serre quotient. Motivated by the above, and also the fact that when studying curves it should not matter how we embed them into surfaces (and thus be independent of any self-intersection numbers that appear), we then investigate when $\mathrm{qgr}^{\mathbb{Z}} S^{\vec{x}} \simeq \operatorname{coh} \mathbb{X}$.

In very special cases, $\operatorname{coh} \mathbb{X}_{\mathbf{p}, \lambda}$ is already known to be equivalent to $\mathrm{qgr}^{\mathbb{Z}} R$ for some connected graded commutative ring $R$ [GL2, 8.4]. The nicest situation is when the star-shaped dual graph is of Dynkin type, and further $R$ is the ADE quotient singularity associated to the Dynkin diagram via the McKay 
correspondence (with a slightly non-standard grading). However, all the previous attempts to link the weighted projective line to rational singularities have taken all self-intersection numbers to be -2 , which is well-known to restrict the possible configurations to ADE Dynkin type.

One of our main results is the following, which does not even require that $\vec{x} \in \mathbb{L}_{+}$.

Theorem 1.9 (=4.7). Suppose that $\vec{x}=\sum_{i=1}^{n} a_{i} \vec{x}_{i}+a \vec{c}$ is not torsion, and write $R:=S^{\vec{x}}$. Then the following conditions are equivalent.

(1) The natural functor $(-)^{\vec{x}}: \mathrm{CM}^{\mathbb{L}} S \rightarrow \mathrm{CM}^{\mathbb{Z}} R$ is an equivalence.

(2) The natural functor $(-)^{\vec{x}}: \mathrm{qgr}^{\mathbb{L}} S \rightarrow \mathrm{qgr}^{\mathbb{Z}} R$ is an equivalence.

(3) For any $\vec{z} \in \mathbb{L}$, the ideal $I^{\vec{z}}:=S(\vec{z})^{\vec{x}} \cdot S(-\vec{z})^{\vec{x}}$ of $R$ satisfies $\operatorname{dim}_{\mathbb{k}}\left(R / I^{\vec{z}}\right)<\infty$.

(4) $\left(p_{i}, a_{i}\right)=1$ for all $1 \leq i \leq n$.

The above theorem implies that for a non-torsion element $\vec{x}=\sum_{i=1}^{n} a_{i} \vec{x}_{i}+a \vec{c}$ of $\mathbb{L}$, there is an equivalence $\operatorname{qgr}^{\mathbb{Z}} S_{\mathbf{p}, \lambda}^{\vec{x}} \simeq \operatorname{coh} \mathbb{X}_{\mathbf{p}, \lambda}$ if and only if $\left(p_{i}, a_{i}\right)=1$ for all $i$ with $1 \leq i \leq n$. Thus, by choosing a suitable $\vec{x}$, the weighted projective line can be defined using only connected $\mathbb{N}$-graded rational surface singularities. Also, we remark that in the case $\left(p_{i}, a_{i}\right) \neq 1$ we still have that $\operatorname{qgr} S^{\vec{x}}$ is equivalent to some weighted projective line, but the parameters are no longer $(\mathbf{p}, \lambda)$. We leave the details to $\S 4$.

Combining the above gives our next main result.

Corollary $1.10(=4.7,4.22)$. Let $\vec{x} \in \mathbb{L}_{+}$with $\vec{x} \notin[0, \vec{c}]$, and write $\vec{x}=\sum_{i=1}^{n} a_{i} \vec{x}_{i}+a \vec{c}$ in normal form. If $\left(p_{i}, a_{i}\right)=1$ for all $1 \leq i \leq n$, then

$$
\operatorname{coh} \mathbb{X}_{\mathbf{p}, \lambda} \simeq \operatorname{qgr}^{\mathbb{Z}} S^{\vec{x}} \simeq \operatorname{qgr}^{\mathbb{Z}} \Gamma_{\vec{x}},
$$

and further $\Gamma_{\vec{x}}$ is an $\mathbb{N}$-graded ring, with zeroth piece a canonical algebra.

In the case when $\left(p_{i}, a_{i}\right) \neq 1$ we have a similar result but again there is a change of parameters, so we refer the reader to 4.22 for details. Combining 1.10 with $1.8(2)$, we can view the weighted projective line $\mathbf{X}_{\mathbf{p}, \lambda}$ as an Artin-Zhang noncommutative projective scheme over the canonical algebra $\Lambda_{\mathrm{p}, \lambda}[\mathrm{M}]$.

Note that $1.9(2) \Leftrightarrow(4)$ was shown independently in [CCZ, 6.6].

\subsection{Some Particular Veronese Subrings}

We then investigate the particular Veronese subrings $S^{\vec{s}_{a}}$ for $\vec{s}_{a}:=\vec{s}+a \vec{c}$ for some $a \geq 0$, where $\vec{s}:=\sum_{i=1}^{n} \vec{x}_{i}$. We call $S^{\overrightarrow{s_{a}}}$ the $a$-Wahl Veronese subring, and in this case, the singularities in (1.B) are all of the form $\frac{1}{p_{i}}(1,-1)$, which are cyclic Gorenstein and so have a resolution consisting of only $(-2)$-curves. Thus resolving the singularities in (1.B), by 1.5 we see that the dual graph of the minimal resolution of Spec $S^{\vec{s}_{a}}$ is

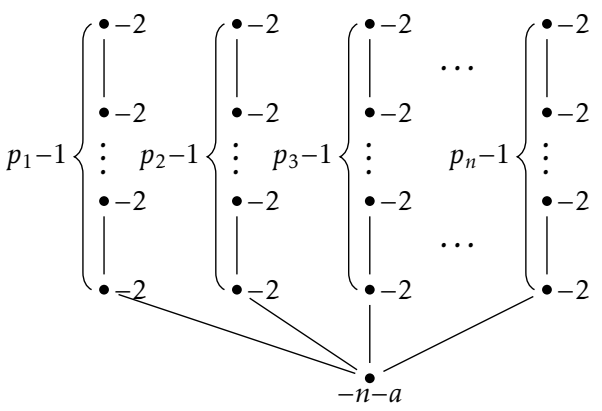

where there are $n$ arms, and the number of vertices on arm $i$ is $p_{i}-1$. It turns out that these particular Veronese subrings have many nice properties; not least by 1.4 they are precisely the Veronese subrings for which

$$
\mathrm{D}^{\mathrm{b}}\left(\operatorname{coh} Y^{\vec{x}}\right) \hookrightarrow \mathrm{D}^{\mathrm{b}}\left(\operatorname{coh} \mathbb{T}^{\vec{x}}\right)
$$


is an equivalence. In $\S 6$ we investigate $S^{\vec{s}_{a}}$ in the case when $\left(p_{1}, p_{2}, p_{3}\right)$ forms a Dynkin triple, in which case $S^{\vec{s}_{a}}$ is isomorphic to a quotient singularity by some finite subgroup of $\mathrm{GL}(2, \mathbb{k})$ of type $\mathbb{T}, \mathbb{O}$ or $\mathbb{I}$ (see 6.1 for details). In this situation $S^{\overrightarrow{s_{a}}}$ and its reconstruction algebra have a very nice relationship to the preprojective algebra of the canonical algebra, and this is what turns out to explain the motivating coincidence from $§ 1.1$ in 1.15 below.

For arbitrary parameters $(\mathbf{p}, \lambda)$, the Veronese subring $S^{\vec{s}}$ has a particularly nice form.

Theorem $1.11(=5.2)$. For any $\mathrm{X}_{\mathrm{p}, \lambda}, S^{\vec{s}}$ is generated by the homogeneous elements

$$
\begin{aligned}
\mathrm{u}_{i}:= \begin{cases}x_{1}^{p_{1}+p_{2}} x_{3}^{p_{2}} \ldots x_{n}^{p_{2}} & i=1, \\
x_{2}^{p_{1}+p_{2}} x_{3}^{p_{1}} \ldots x_{n}^{p_{1}} & i=2, \\
-x_{1}^{p_{i}} x_{2}^{p_{2}+p_{i}} x_{3}^{p_{i}} \ldots \widehat{x_{i}} \ldots x_{n}^{p_{i}} & 3 \leq i \leq n,\end{cases} \\
\mathrm{v}:=x_{1} x_{2} \ldots x_{n} .
\end{aligned}
$$

Proposition $1.12(=5.5)$. With notation as above, the modules $S\left(u \vec{x}_{j}\right)^{\vec{s}}$ appearing in 1.7 are precisely the following ideals of $S^{\vec{s}}$, and furthermore they correspond to the dual graph of the minimal resolution of $\mathrm{Spec} S^{\vec{s}}(1 . \mathrm{F})$ in the following way:

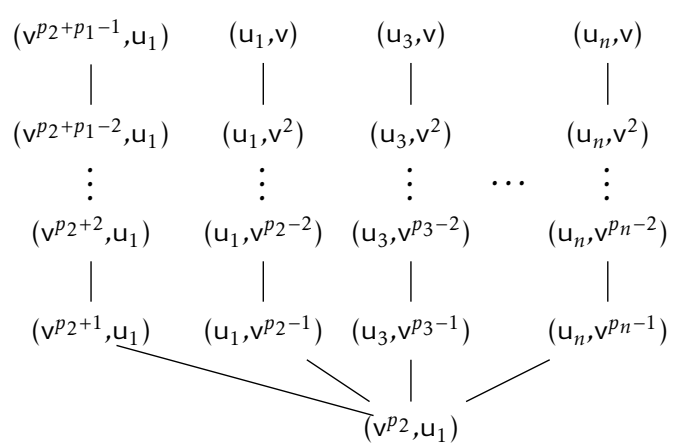

The relations between $\mathrm{u}_{1}, \ldots, \mathrm{u}_{n}, \mathrm{v}$ turn out to be easy to describe, and remarkably have already appeared in the literature. It is well-known [W1,3.6] that there is a family of rational surface singularities $R_{\mathbf{p}, \lambda}$ where the dual graph of the minimal resolution of $\operatorname{Spec} R_{\mathbf{p}, \lambda}$ is precisely (2.A) with $a=0$. Indeed, in [W1] $R_{\mathbf{p}, \lambda}$ is defined as follows: given the same data $(\mathbf{p}, \lambda)$ as above normalised so that $\lambda_{1}=(1: 0), \lambda_{2}=(0: 1)$ and $\lambda_{3}, \ldots, \lambda_{n} \in \mathbb{k}^{*}$ are pairwise distinct, we can consider the commutative $\mathbb{k}$-algebra $R_{\mathbf{p}, \lambda}$, generated by $u_{1}, \ldots, u_{n}, v$ subject to the relations given by the $2 \times 2$ minors of the matrix

$$
\left(\begin{array}{ccccc}
u_{2} & u_{3} & \ldots & u_{n} & v^{p_{2}} \\
v^{p_{1}} & \lambda_{3} u_{3}+v^{p_{3}} & \ldots & \lambda_{n} u_{n}+v^{p_{n}} & u_{1}
\end{array}\right)
$$

This is a connected $\mathbb{N}$-graded ring graded by $\operatorname{deg} v:=1, \operatorname{deg} u_{1}:=p_{2}, \operatorname{deg} u_{2}:=p_{1}$ and $\operatorname{deg} u_{i}:=p_{i}$ for all $3 \leq i \leq n$.

We show that $S^{\vec{s}}$ recovers precisely the above $R_{\mathbf{p}, \lambda}$.

Theorem 1.13 (=5.3). There is an isomorphism $R_{\mathbf{p}, \lambda} \cong S^{\vec{s}}$ of $\mathbb{Z}$-graded algebras given by $u_{i} \mapsto \mathrm{u}_{i}$ for $1 \leq i \leq n$ and $v \mapsto \mathrm{v}$.

Thus the Veronese method we develop in this paper for constructing rational surface singularities recovers as a special case the example of [W1], but in a way suitable for arbitrary labelled star-shaped graphs, and also in a way suitable for obtaining the special CM modules.

We then present the reconstruction algebra of $R_{\mathbf{p}, \lambda} \cong S^{\vec{s}}$, since again in this situation it has a particularly nice form. In principle, using 1.7 , we can do this for any Veronese $S^{\vec{x}}$ with $0 \neq \vec{x} \in \mathbb{L}_{+}$, but for notational ease we restrict ourselves to the case $\vec{x}=\vec{s}$. 
Theorem 1.14 (=5.7). The reconstruction algebra $\Gamma_{\mathbf{p}, \lambda}$ of $R_{\mathbf{p}, \lambda}$ can be written explicitly as a quiver with relations. It is the path algebra of the double of the quiver $Q_{\mathbf{p}}$ of the canonical algebra, subject to the relations induced by the canonical relations, and furthermore at every vertex, all 2-cycles that exist at that vertex are equal.

We refer the reader to 5.7 for more details, but remark that the reconstruction algebra was originally invented in order to extend the notion of a preprojective algebra to a more general geometric setting. In our situation here, the reconstruction algebra is not quite the preprojective algebra of the canonical algebra $\Lambda_{\mathbf{p}, \lambda}$, but the relations in 5.7 are mainly of the same form as the preprojective relations; the reconstruction algebra should perhaps be thought of as a better substitute.

In the last section of the paper, finally we can explain the coincidence of the two motivating pictures, as a consequence of the following result.

Theorem 1.15 (=6.3). Let $R$ be the (m-3)-Wahl Veronese subring associated with $\left(p_{1}, p_{2}, p_{3}\right)=(2,3,3),(2,3,4)$ or $(2,3,5)$ and $m \geq 3$, and $\mathcal{R}$ its completion. Let $G \leq \mathbb{L}$ be the cyclic group generated by $(h(m-2)+1) \vec{\omega}$, where $h=6,12$ or 30 respectively. Then

(1) There are equivalences vect $\mathrm{X} \simeq \mathrm{CM}^{\mathbb{Z}} R$ and

$$
F:(\operatorname{vect} X) / G \stackrel{\sim}{\rightarrow} \mathrm{CMR},
$$

where $(\operatorname{vect} \mathbb{X}) / G$ is the complete orbit category (for the definition, see $\$ 6)$.

(2) For the canonical tilting bundle $\mathcal{E}$ on $\mathrm{X}$, we have $\mathrm{SCM} \mathcal{R}=\operatorname{add} F \mathcal{E}$.

Acknowledgements. The authors thank Kazushi Ueda and Atsushi Takahashi for many helpful comments and remarks, and Mitsuyasu Hashimoto, Ryo Takahashi and Yuji Yoshino for valuable discussions on reflexive modules. Part of this work was completed when O.I. visited Edinburgh in March and September 2012, and he thanks people in Edinburgh for hospitality during his visit.

Conventions. Throughout, $\mathbb{k}$ denotes an algebraically closed field of characteristic zero. All modules will be right modules, and for a ring $A$ write $\bmod A$ for the category of finitely generated right $A$-modules. If $G$ is an abelian group and $A$ is a noetherian $G$-graded $\operatorname{ring}, \mathrm{gr}^{G} A$ will denote the category of finitely generated $G$-graded right $A$-modules. Throughout when composing maps $f g$ will mean $f$ then $g$, similarly for arrows $a b$ will mean $a$ then $b$. Note that with this convention $\operatorname{Hom}_{R}(M, N)$ is an $\operatorname{End}_{R}(M)^{\mathrm{op}}$-module and an $\operatorname{End}_{R}(N)$-module. For $M \in \bmod A$ we write $\operatorname{add} M$ for the full subcategory consisting of summands of finite direct sums of copies of $M$.

\section{Preliminaries}

\subsection{Notation}

We first fix notation. For $n \geq 0$, choose positive integers $p_{1}, \ldots, p_{n}$ with all $p_{i} \geq 2$, and set $\mathbf{p}:=\left(p_{1}, \ldots, p_{n}\right)$. Likewise, for pairwise distinct points $\lambda_{1}, \ldots, \lambda_{n} \in \mathbb{P}^{1}$, set $\lambda:=\left(\lambda_{1}, \ldots, \lambda_{n}\right)$. Let $\ell_{i}\left(t_{0}, t_{1}\right) \in \mathbb{k}\left[t_{0}, t_{1}\right]$ be the linear form defining $\lambda_{i}$.

Notation 2.1. To this data we associate the following.

(1) The abelian group $\mathbb{L}=\mathbb{L}\left(p_{1}, \ldots, p_{n}\right)$ generated by the elements $\vec{x}_{1}, \ldots, \vec{x}_{n}$ subject to the relations $p_{1} \vec{x}_{1}=p_{2} \vec{x}_{2}=\cdots=p_{n} \vec{x}_{n}=: \vec{c}$. Note that $\mathbb{L}$ is an ordered group with $\mathbb{L}_{+}=\sum_{i=1}^{n} \mathbb{Z}_{\geq 0} \vec{x}_{i}$ as positive elements . Since $\mathbb{L} / \mathbb{Z} \vec{c} \cong \prod_{i=1}^{n} \mathbb{Z} / p_{i} \mathbb{Z}$ canonically, each $\vec{x} \in \mathbb{L}$ can be written uniquely in normal form as $\vec{x}=\sum_{i=1}^{n} a_{i} \vec{x}_{i}+a \vec{c}$ with $0 \leq a_{i}<p_{i}$ and $a \in \mathbb{Z}$. Then $\vec{x}$ belongs to $\mathbb{L}_{+}$if and only if $a \geq 0$. The dualizing element $\vec{\omega} \in \mathbb{L}$ is defined to be

$$
\vec{\omega}:=(n-2) \vec{c}-\sum_{i=1}^{n} \vec{x}_{i} .
$$


(2) The commutative $\mathbb{k}$-algebra $S_{\mathbf{p}, \lambda}$ defined as

$$
S_{\mathbf{p}, \lambda}=S:=\frac{\mathbb{k}\left[t_{0}, t_{1}, x_{1}, \ldots, x_{n}\right]}{\left(x_{i}^{p_{i}}-\ell_{i}\left(t_{0}, t_{1}\right) \mid 1 \leq i \leq n\right)} .
$$

As in the introduction, this is $\mathbb{L}$-graded by $\operatorname{deg} x_{i}:=\vec{x}_{i}$, and defines the weighted projective line $\mathbb{X}_{\mathbf{p}, \lambda}:=[(\operatorname{Spec} S \backslash 0) / \operatorname{Spec} \mathbb{k} \mathbb{L}]$. Then its coarse moduli space $X_{\mathbf{p}, \lambda}:=(\operatorname{Spec} S \backslash 0) / \operatorname{Spec} \mathbb{k} \mathbb{L}$ is $\mathbb{P}^{1}$. In fact, the open cover Spec $S \backslash 0=U_{0} \cup U_{1}$ with $U_{i}:=\operatorname{Spec} S_{t_{i}}$ induces an open cover $X_{\mathbf{p}, \lambda}=X_{0} \cup X_{1}$ with $X_{i}:=\operatorname{Spec}\left(S_{t_{i}}\right)_{0}$, where $\left(S_{t_{i}}\right)_{0}$ is the degree zero part of $S_{t_{i}}$. By inspection $\left(S_{t_{i}}\right)_{0}=\mathbb{k}\left[t_{1-i} / t_{i}\right]$, and it follows that $X_{\mathbf{p}, \lambda} \cong \mathbb{P}^{1}$.

When $n \geq 2$, often we choose $\lambda_{1}=(1: 0)$ and $\lambda_{2}=(0: 1)$, in which case $\ell_{1}=t_{1}, \ell_{2}=t_{0}$ and $\ell_{i}=\lambda_{i} t_{0}-t_{1}$ for $3 \leq i \leq n$, and there is a presentation

$$
S_{\mathbf{p}, \lambda}=\frac{\mathbb{k}\left[x_{1}, \ldots, x_{n}\right]}{\left(x_{i}^{p_{i}}+x_{1}^{p_{1}}-\lambda_{i} x_{2}^{p_{2}} \mid 3 \leq i \leq n\right)} .
$$

Moreover, when $n \geq 2$, we can further associate

(3) The quiver

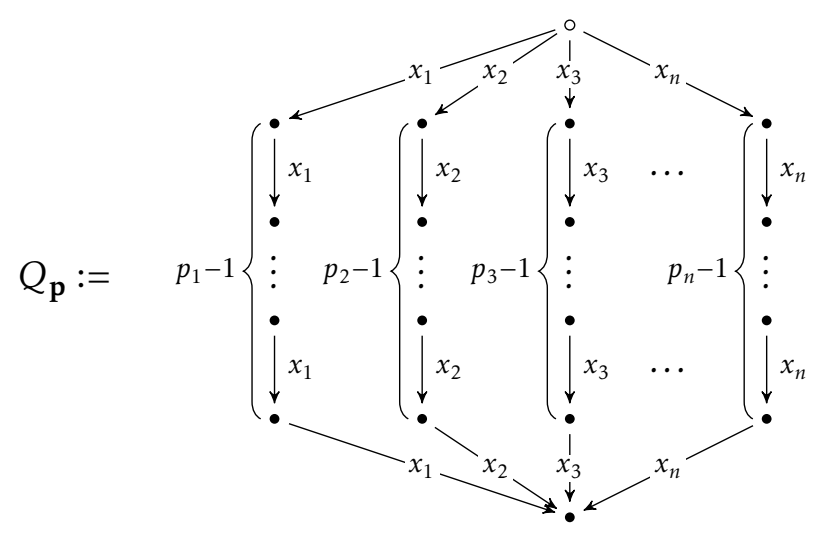

(where there are $n$ arms, and the number of vertices on arm $i$ is $p_{i}-1$ ).

(4) The canonical algebra $\Lambda_{\mathbf{p}, \lambda}$, namely the path algebra of the quiver $Q_{\mathbf{p}}$ subject to the relations

$$
I:=\left\langle x_{1}^{p_{1}}-\lambda_{i} x_{2}^{p_{2}}+x_{i}^{p_{i}} \mid 3 \leq i \leq n\right\rangle .
$$

There is a degenerate definition of the canonical algebra if $0 \leq n \leq 1$; see [GL1].

(5) The commutative $\mathbb{k}_{\text {-algebra }} R_{\mathbf{p}, \lambda}$, generated by $u_{1}, \ldots, u_{n}, v$ subject to the relations given by the $2 \times 2$ minors of the matrix

$$
\left(\begin{array}{ccccc}
u_{2} & u_{3} & \ldots & u_{n} & v^{p_{2}} \\
v^{p_{1}} & \lambda_{3} u_{3}+v^{p_{3}} & \ldots & \lambda_{n} u_{n}+v^{p_{n}} & u_{1}
\end{array}\right)
$$

This is a connected $\mathbb{N}$-graded ring graded by $\operatorname{deg} u_{1}:=p_{2}, \operatorname{deg} u_{2}:=p_{1}, \operatorname{deg} v:=1$, and $\operatorname{deg} u_{i}:=p_{i}$ for all $3 \leq i \leq n$.

We will also consider 
(6) Star-shaped graphs of the form

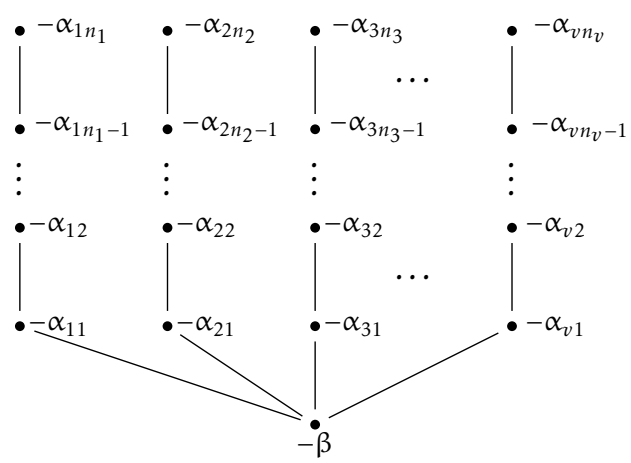

where there are $v \geq 2$ arms, each $n_{i} \geq 1$, each $\alpha_{i j} \geq 2$ and $\beta \geq 1$. Later, we will assume $\beta \geq v$.

We next give some properties of $S_{\mathbf{p}, \lambda}$ and related rings that will be required later, all being elementary in nature. We start with a general result. Let $G$ be an abelian group and $A$ a $G$-graded ring. Then $A$ is a G-domain whenever a product of non-zero homogeneous elements is again non-zero, and a G-field if any non-zero homogeneous element is invertible. A homogeneous element $x \in A$ is $G$-prime if $A /(x)$ is an $G$-domain. If $A$ is a $G$-domain, then the quotient $G$-field of $A$ is the localization of $A$ with respect to the set of all non-zero homogeneous elements. A $G$-domain is $G$-factorial if every non-zero homogeneous element in $A$ is a product of $G$-prime elements in $A$.

Proposition 2.2. With notation as above,

(1) $S_{\mathbf{p}, \lambda}$ is an $\mathbb{L}$-factorial $\mathbb{L}$-domain.

(2) Any G-factorial G-domain A is G-normal, i.e. if a homogeneous element $x$ in the quotient $G$-field of $A$ satisfies an equality $x^{m}+a_{1} x^{m-1}+\cdots+a_{m}=0$ for some $a_{i} \in A$, then $x \in A$. In particular, $A_{0}$ is a normal domain (in the usual sense).

(3) Let $A$ be a G-field and $A[y]$ the G-graded polynomial ring with a homogeneous indeterminate $y$. Then any homogeneous ideal of $A[y]$ is principal.

(4) If A is a G-factorial G-domain, the localization of A by a set of homogeneous elements is G-factorial. The G-graded polynomial ring $A\left[y_{1}, \ldots, y_{m}\right]$ with homogeneous indeterminates $y_{1}, \ldots, y_{m}$ is also G-factorial.

Proof. Part (1) is well known, see e.g. [GL1, 1.3] or [HIMO, Section 2.2]. Parts (2), (3) and the first assertion of (4) are easy. The second assertion of (4) follows from a parallel argument to the classical case [B4, Section VII.3.5] using (3) and the G-version of Gauss's Lemma.

Let $S=S_{\mathbf{p}, \lambda}$. Recall from the introduction that for $\vec{x} \in \mathbb{L}, S^{\vec{x}}:=\bigoplus_{i \in \mathbb{Z}} S_{i \vec{x}}$. We will also be interested in the $\mathbb{N}$-graded version, so define $S^{\mathbb{N} \vec{x}}:=\bigoplus_{i \geq 0} S_{i \vec{x}}$.

Corollary 2.3. With notation as above, let $\vec{x} \in \mathbb{L}$.

(1) If $\vec{x} \in \mathbb{L}$ is not torsion, then $S^{\vec{x}}$ is a noetherian $\mathbb{k}$-algebra with $\operatorname{dim} S^{\vec{x}}=2$, and $S$ is a finitely generated $S^{\vec{x}}$-module.

(2) Let $S[t]$ be the $\mathbb{L}$-graded polynomial ring with $\operatorname{deg} t=-\vec{x}$. Then $(S[t])_{0} \cong S^{\mathbb{N} \vec{x}}$ holds, and this is a normal domain.

(3) Suppose $-i \vec{x} \notin \mathbb{L}_{+}$for all $i>0$. Then $S^{\vec{x}}$ is a noetherian normal domain with $\operatorname{dim} S^{\vec{x}}=2$, and has at worst a unique singular point corresponding to $\bigoplus_{i>0} S_{i \vec{x}}$. 
Proof. (1) Since $\vec{x}$ is not torsion, $\mathbb{Z} \vec{x} \subseteq \mathbb{L}$ has finite index, and so the first two assertions of (1) are easy; see e.g. 4.2. In particular, necessarily $\operatorname{dim} S^{\vec{x}}=\operatorname{dim} S=2$.

(2) The equality $(S[t])_{0}=\bigoplus_{i \geq 0} S_{i \vec{x}} t^{i} \cong S^{\mathbb{N} \vec{x}}$ is clear. Furthermore, $S[t]$ is an $\mathbb{L}$-factorial $\mathbb{L}$-domain according to $2.2(1)(4)$. Thus $(S[t])_{0}$ is a normal domain by $2.2(2)$.

(3) The assumption $-i \vec{x} \notin \mathbb{L}_{+}$for all $i>0$ implies that $\vec{x}$ is not torsion, since otherwise $-N x=0 \in \mathbb{L}_{+}$for some $N>0$. It also forces $S^{\vec{x}}=S^{\mathbb{N} \vec{x}}$, so the first half of the result follows by combining parts (1) and (2).

The second half is a general property of a positively graded two-dimensional normal domain (e.g. [P, p1]). In fact, since $S^{\vec{x}}$ is a $\mathbb{Z}$-graded finitely generated $\mathbf{k}$-algebra, by the Jacobian criterion, there is a $\mathbb{Z}$ graded ideal $I$ of $S^{\vec{x}}$ such that $\operatorname{Sing} S^{\vec{x}}=\operatorname{Spec}\left(S^{\vec{x}} / I\right)$. Since $S^{\vec{x}}$ is normal, all singularities of $S^{\vec{x}}$ are isolated and $\operatorname{dim}_{\mathbb{k}}\left(S^{\vec{x}} / I\right)<\infty$ holds. Since $I$ is $\mathbb{Z}$-graded, it contains $\bigoplus_{i>\ell} S_{i \vec{x}}$ for $\ell \gg 0$ and hence $\sqrt{I}$ contains $\bigoplus_{i>0} S_{i \vec{x}}$. Thus Sing $S^{\vec{x}} \subseteq\left\{\bigoplus_{i>0} S_{i \vec{x}}\right\}$.

The following will be required later, and all are well known (see [GL1]).

Lemma 2.4. If $\vec{x}=\sum_{i=1}^{n} a_{i} \vec{x}+a \vec{c} \in \mathbb{L}_{+}$is in normal form, then the following hold.

(1) $S_{\vec{x}}=\left(\prod_{i=1}^{n} x_{i}^{a_{i}}\right) S_{a \vec{c}}$.

(2) $S_{a \vec{c}}$ is an $(a+1)$-dimensional vector space, and a basis of $S_{a \vec{c}}$ is given by $t_{0}^{\ell} t_{1}^{a-\ell}$ with $0 \leq \ell \leq a$.

(3) $S_{\vec{x}+m \vec{c}}=S_{\vec{x}} \cdot S_{m \vec{c}}$ for all $m \geq 0$.

\subsection{Preliminaries on Rational Surface Singularities}

We briefly review some combinatorics associated to rational surface singularities. Let $R$ be a finitely generated noetherian $\mathbb{k}$-algebra, or alternatively the completion of such an algebra at a maximal ideal. Recall that $R$ is said to be a rational surface singularity if $\operatorname{dim} R=2$ and there exists $f: X \rightarrow \operatorname{Spec} R$ a resolution such that $\mathbf{R} f_{*} \mathcal{O}_{X}=\mathcal{O}_{R}$. If this property holds for one resolution, it holds for all resolutions [KM, 5.10], and automatically $R$ must be normal [KM, 5.8].

In our setting later $R$ will be a rational surface singularity with a unique singular point, at the origin. Completing at this maximal ideal to give $\mathfrak{K}$, in the minimal resolution $Y \rightarrow \operatorname{Spec} \mathcal{K}$ the fibre above the origin is well-known to be a tree (i.e. a finite connected graph with no cycles) of $\mathbb{P}^{1}$ s denoted $\left\{E_{i}\right\}_{i \in I}$. Their self-intersection numbers satisfy $E_{i} \cdot E_{i} \leq-2$, and moreover the intersection matrix $\left(E_{i} \cdot E_{j}\right)_{i, j \in I}$ is negative definite. We encode the intersection matrix in the form of the labelled dual graph:

Definition 2.5. Suppose that $\left\{E_{i}\right\}_{i \in I}$ is a collection of $\mathbb{P}^{1} s$ forming the exceptional locus in a resolution of some rational surface singularity. The dual graph is defined as follows: for each curve $E_{i}$ there is a vertex, with $E_{i} \cdot E_{j}$ edges connecting the vertices corresponding to $E_{i}$ and $E_{j}$. Furthermore, every vertex is labelled with the self-intersection number of the corresponding curve.

The dual graph of a complete local rational surface singularity is well known to be a labelled tree (see e.g. [B3, 1.3]). Conversely, suppose that $T$ is a tree, with vertices denoted $E_{1}, \ldots, E_{n}$, labelled with integers $w_{1}, \ldots, w_{n}$. To this data we associate the symmetric matrix $M_{T}=\left(b_{i j}\right)_{1 \leq i, j \leq n}$ with $b_{i i}$ defined by $b_{i i}:=w_{i}$, and $b_{i j}$ (with $i \neq j$ ) defined to be the number of edges linking the vertices $E_{i}$ and $E_{j}$. We write $\mathcal{Z}$ for the free abelian group generated by the vertices $E_{i}$, and call its elements cycles. The matrix $M_{T}$ defines a symmetric bilinear form $(-,-)$ on $\mathcal{Z}$ and in analogy with the geometry, we will often write $Y \cdot Z$ instead of $(Y, Z)$, and consider

$$
\mathcal{Z}_{\text {top }}:=\left\{Z=\sum_{i=1}^{n} a_{i} E_{i} \in \mathcal{Z} \mid Z \neq 0 \text {, all } a_{i} \geq 0 \text {, and } Z \cdot E_{i} \leq 0 \text { for all } i\right\} .
$$

If there exists $Z \in \mathcal{Z}_{\text {top }}$ such that $Z \cdot Z<0$, then automatically $M_{T}$ is negative definite [A, Prop 2(ii)]. In this case, $\mathcal{Z}_{\text {top }}$ admits a unique smallest element $Z_{f}$, called the fundamental cycle. Whenever all the coefficients in $Z_{f}$ are one, the fundamental cycle is said to be reduced. 
We now consider the case of the labelled graph (2.A) and calculate some combinatorics that will be needed later. Denoting the set of vertices of (2.A) by $I$, considering $Z:=\sum_{i \in I} E_{i}$ it is easy to see that

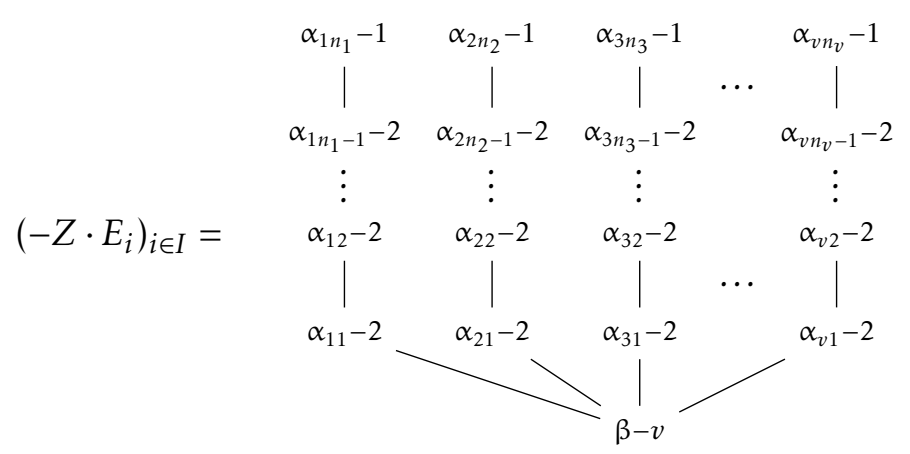

and so $Z$ satisfies $Z \cdot E_{i} \leq 0$ for all $i \in I$ if and only if $\beta \geq v$. Since $\mathcal{Z}_{\text {top }}$ does not contain elements smaller than $Z$, the fundamental cycle $Z_{f}$ is given by $Z=\sum_{i \in I} E_{i}$ if and only if $\beta \geq v$. In this case $Z_{f}$ is reduced.

We remark that in general there will be many singularities with dual graph (2.A), and indeed a labelled graph $T$ is called taut if there exists a unique rational surface singularity (up to isomorphism in the category of complete local $\mathrm{k}$-algebras) which has $T$ for its dual graph of its minimal resolution. It is well known that the labelled graph (2.A) is taut if and only if $v=3[\mathrm{~L}]$.

\subsection{Preliminaries on Reconstruction Algebras}

Let $R$ be a rational surface singularity. A CM $R$-module $M$ is called special if $\operatorname{Ext}_{R}^{1}(M, R)=0$ [IW], and we write $\mathrm{SCM} R$ for the category of special CM $R$-modules.

The following local-to-global lemma is useful. In particular, if $R$ has a unique singular point $\mathrm{m}$, to conclude that add $M=\mathrm{SCM} R$ it suffices to check this complete locally at $\mathrm{m}$.

Lemma 2.6. Let $R$ be a rational surface singularity, and $M \in \mathrm{CM} R$. If add $\widehat{M}_{\mathrm{m}}=\mathrm{SCM} \widehat{R}_{\mathrm{m}}$ for all $\mathrm{m} \in \mathrm{Max} R$, then add $M=\mathrm{SCM} R$.

Proof. Since Ext groups localise and complete, certainly $M \in \mathrm{SCM} R$ and thus add $M \subseteq \mathrm{SCM} R$. Next, let $X \in \operatorname{SCM} R$. Then add $\widehat{X}_{\mathrm{m}} \subseteq \operatorname{SCM} \widehat{R}_{\mathrm{m}}$ for all $\mathrm{m} \in \operatorname{Max} R$, so by assumption add $\widehat{X}_{\mathrm{m}} \subseteq \operatorname{add} \widehat{M}_{\mathrm{m}}$ for all $\mathrm{m} \in \operatorname{Max} R$. By [IW2, 2.26] we conclude that $\operatorname{add} X \subseteq \operatorname{add} M$, so $X \in \operatorname{add} M$ and thus add $M \supseteq \mathrm{SCM} R$.

The following asserts that a global additive generator of SCMR exists, regardless of the number of points in the singular locus.

Theorem 2.7 ([VdB]). Let $R$ be a rational surface singularity, and $\pi: X \rightarrow \operatorname{Spec} R$ the minimal resolution. Then the following statements hold.

(1) There exists $M \in \mathrm{SCM} R$ such that $\mathrm{SCM} R=\operatorname{add} M$.

(2) There is a triangle equivalence $\mathrm{D}^{\mathrm{b}}\left(\operatorname{modEnd} \mathrm{E}_{R}(M)\right) \cong \mathrm{D}^{\mathrm{b}}(\operatorname{coh} X)$.

Proof. This is known but usually only stated when $R$ is complete, so for the convenience of the reader we provide a proof. By $[\mathrm{VdB}, 3.2 .5]$ there is a progenerator $\mathcal{O}_{X} \oplus \mathcal{M}$ for the category of perverse sheaves (with perversity -1 ), which induces an equivalence

$$
\mathrm{D}^{\mathrm{b}}\left(\operatorname{modEnd}{ }_{X}\left(\mathcal{O}_{X} \oplus \mathcal{M}\right)\right) \cong \mathrm{D}^{\mathrm{b}}(\operatorname{coh} X) .
$$

There is an isomorphism $\operatorname{End}_{X}\left(\mathcal{O}_{X} \oplus \mathcal{M}\right) \cong \operatorname{End}_{R}\left(R \oplus \pi_{*} \mathcal{M}\right)$ by [DW, 4.1]. Furthermore, $\mathcal{O}_{X} \oplus \mathcal{M}$ remains a progenerator under flat base change $[\mathrm{VdB}, 3.1 .6]$, so add $\widehat{M}_{\mathrm{m}}=\mathrm{SCM} \widehat{R}_{\mathrm{m}}$ by [W6, IW]. The result then follows using 2.6 . 
Definition 2.8. For any $M \in \operatorname{SCM} R$ such that $\operatorname{SCM} R=\operatorname{add} M$, we call $\operatorname{End}_{R}(M)$ the reconstruction algebra.

In this global setting, the reconstruction algebra is only defined up to Morita equivalence. Only after completing $R$, or in certain other settings (see 2.11) will there be a canonical choice.

When $\mathcal{K}$ is a complete local rational surface singularity with minimal resolution $X \rightarrow$ Spec $\mathcal{K}$, there is a much more explicit description of the additive generator of SCM $R$. Let $\left\{E_{i} \mid i \in I\right\}$ denote the irreducible exceptional curves, then for each $i \in I$, by [W6] there exists a CM $\mathcal{K}$-module $M_{i}$ such that $H^{1}\left(\mathcal{M}_{i}^{\vee}\right)=0$ and $c_{1}\left(\mathcal{M}_{i}\right) \cdot E_{j}=\delta_{i j}$ hold, where $\mathcal{M}_{i}:=\pi^{*}\left(M_{i}\right)^{\vee \vee}$ for $(-)^{\vee}=\mathcal{H o m}_{X}\left(-, \mathcal{O}_{X}\right)$.

Theorem 2.9 ([W6, 1.2]). There is a bijection

$\{$ irreducible exceptional curves in min. resolution $\} \longleftrightarrow\{$ non-free, indecomposable special CM $\mathcal{R}$-modules $\}$

$$
E_{i} \longmapsto M_{i} \text {. }
$$

Furthermore, the rank of $M_{i}$, as an $\mathcal{R}$-module, coincides with the co-efficient of $E_{i}$ in $Z_{f}$.

It follows that $\mathfrak{R} \oplus \bigoplus_{i \in I} M_{i}$ is the natural additive generator for SCM $\mathcal{R}$.

Definition 2.10. Let $\mathfrak{K}$ be a complete local rational surface singularity. We call $\Gamma:=\operatorname{End}_{\mathfrak{K}}\left(\mathcal{K} \oplus\left(\bigoplus_{i \in I} M_{i}\right)\right)$ the reconstruction algebra of $\mathcal{R}$.

Remark 2.11. If $R$ is a rational surface singularity with a unique singular point, and if there exist $L_{i} \in \mathrm{CM} R$ such that $\widehat{L}_{i} \cong M_{i}$ for all $i$, then we also use the letter $\Gamma$ to denote the particular reconstruction algebra

$$
\Gamma:=\operatorname{End}_{R}\left(R \oplus \bigoplus_{i \in I} L_{i}\right)
$$

of $R$. Such $L_{i}$ are not guaranteed to exist, in general.

In the complete local setting, the quiver of $\Gamma$, and the number of its relations, is completely determined by the intersection theory.

Theorem 2.12 ([W2, 3.3]). Let $\mathcal{K}$ be a complete local rational surface singularity. The quiver and the numbers of relations of $\Gamma$ is given as follows: for every $i \in I$ associate a vertex labelled $i$ corresponding to $M_{i}$, and also associate a vertex labelled $\circ$ corresponding to $\mathfrak{k}$. Then the number of arrows and relations between the vertices is

\begin{tabular}{ccc}
\hline & Number of arrows & Number of relations \\
\hline$i \rightarrow j$ & $\left(E_{i} \cdot E_{j}\right)_{+}$ & $\left(-1-E_{i} \cdot E_{j}\right)_{+}$ \\
$\circ \rightarrow \circ$ & 0 & $-Z_{K} \cdot Z_{f}+1=-1-Z_{f} \cdot Z_{f}$ \\
$i \rightarrow \circ$ & $-E_{i} \cdot Z_{f}$ & 0 \\
$\circ \rightarrow i$ & $\left(\left(Z_{K}-Z_{f}\right) \cdot E_{i}\right)_{+}$ & $\left(\left(Z_{K}-Z_{f}\right) \cdot E_{i}\right)_{-}$ \\
\hline
\end{tabular}

where for $a \in \mathbb{Z}$

$$
a_{+}:=\left\{\begin{array}{lll}
a & \text { if } & a \geq 0 \\
0 & \text { if } & a<0
\end{array} \quad \text { and } a_{-}=\left\{\begin{array}{rll}
0 & \text { if } & a \geq 0 \\
-a & \text { if } & a<0
\end{array},\right.\right.
$$

and the canonical cycle $Z_{K}$ is by definition the rational cycle defined by the condition $Z_{K} \cdot E_{i}=E_{i}^{2}+2$ for all $i \in I$. 


\subsection{Hirzebruch-Jung Continued Fraction Combinatorics}

We review briefly the notation and combinatorics surrounding dimension two cyclic quotient singularities.

Definition 2.13. For $r, a \in \mathbb{N}$ with $r>a$ the group $G=\frac{1}{r}(1, a)$ is defined by

$$
G=\left\langle\zeta:=\left(\begin{array}{cc}
\varepsilon & 0 \\
0 & \varepsilon^{a}
\end{array}\right)\right\rangle \leq \mathrm{GL}(2, \mathbb{k}),
$$

where $\mathcal{E}$ is a primitive $r^{\text {th }}$ root of unity. By abuse of notation, we also write $\frac{1}{r}(1, a)$ for the corresponding quotient singularity $\mathrm{k}[x, y]^{G}$.

Remark 2.14. In the literature it is often assumed that the greatest common divisor $(r, a)$ is 1 , which is equivalent to the group having no pseudoreflections. However we do not make this assumption, since in our construction later groups with pseudoreflections naturally appear.

Provided that $a \neq 0$, we consider the Hirzebruch-Jung continued fraction expansion of $\frac{r}{a}$, namely

$$
\frac{r}{a}=\alpha_{1}-\frac{1}{\alpha_{2}-\frac{1}{\alpha_{3}-\frac{1}{(\ldots)}}}:=\left[\alpha_{1}, \ldots, \alpha_{n}\right]
$$

with each $\alpha_{i} \geq 2$. The labelled Dynkin diagram

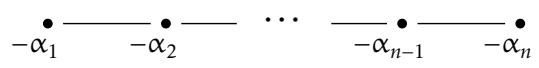

is precisely the dual graph of the minimal resolution of $\mathrm{k}^{2} / \frac{1}{r}(1, a)$ [R1, Satz8]. Note that [R1] assumed the condition $(r, a)=1$, but the result holds generally: if we write $h:=(r, a)$, then the quotient singularities $\frac{1}{r}(1, a)$ and $\frac{1}{r / h}(1, a / h)$ are isomorphic, and furthermore both have the same Hirzebruch-Jung continued fraction expansion.

Definition 2.15. For integers $1 \leq a<r$ as above, consider the continued fraction expansion $\frac{r}{a}=\left[\alpha_{1}, \ldots, \alpha_{n}\right]$. Then the $i$-series is defined as $i_{0}=r, i_{1}=a$ and

$$
i_{t}=\alpha_{t-1} i_{t-1}-i_{t-2}
$$

for all $t$ with $2 \leq t \leq n+1$. Thus $i_{n+1}=0$ holds. Let $I(r, a):=\left\{i_{0}, i_{1}, \ldots, i_{n+1}\right\}$, and by convention $I(r, r):=\emptyset$.

The following lemma is elementary, and will be needed later.

Lemma 2.16. For integers $1 \leq a<r, I(r, a)=[0, r]$ if and only if $a=r-1$.

For a cyclic quotient singularity $G=\frac{1}{r}(1, a)$, consider

$$
S_{t}:=\left\{f \in \mathbb{k}[x, y] \mid \sigma \cdot f=\varepsilon^{t} f\right\},
$$

for $t \in[0, r]$, and note that $S_{0} \cong S_{r}$. Further, for $k$ with $0 \leq k \leq r-1$, we say that a monomial $x^{m} y^{n}$ has weight $k$ if $m+a n=k \bmod r$, that is $x^{m} y^{n} \in S_{k}$. It is the $i$-series that determines which $\mathrm{CM} S^{G}$-modules are special.

Theorem 2.17. For $G=\frac{1}{r}(1, a)$,

(1) [H3] $\mathrm{CM} S^{G}=\operatorname{add}\left\{S_{t} \mid t \in[0, r]\right\}$.

(2) $[\mathrm{W} 5] \operatorname{SCM}^{G}=\operatorname{add}\left\{S_{t} \mid t \in I(r, a)\right\}$. 
Proof. Both results are usually stated in the complete case, with no pseudoreflections, so since we are working more generally, we give the proof. Since $S^{G}$ has a unique singular point, by 2.6 (and its counterpart in the $\mathrm{CM} S^{G}$ case) it suffices to prove both results in the complete local setting. In this case, when $(r, a)=1$, part (1) is [H3] and part (2) is [W5]. When $(r, a) \neq 1$, the result is still true since $\frac{1}{r}(1, a)=\frac{1}{r / h}(1, a / h)$ for $h:=(r, a)$.

In what follows, we will require a different characterization of members of the $i$-series, by reinterpreting a result of Ito [I, 3.7]. As notation, if $(r, a)=1$ then the $G$-basis $B(G)$ is defined to be the set of monomials which are not divisible by any $G$-invariant monomial. We usually draw $B(G)$ in a $2 \times 2$ grid.

Example 2.18. Consider $G=\frac{1}{17}(1,10)$. Then $B(G)$ is

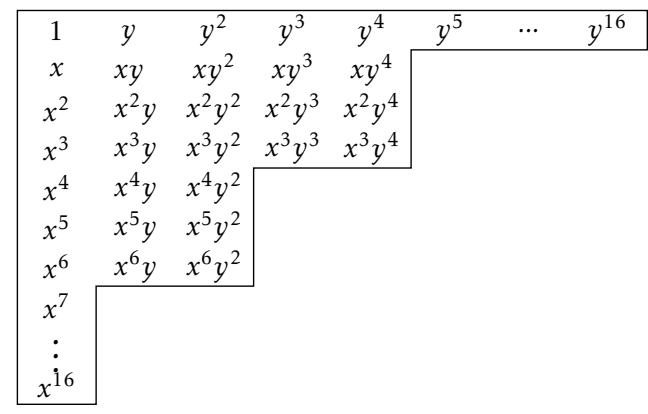

For $G=\frac{1}{r}(1, a)$ with $(r, a)=1$, recall that the $L$-space $L(G)$ is defined to be

$$
L(G):=\left\{1, x, \ldots, x^{r-1}, y, \ldots, y^{r-1}\right\},
$$

so called since in the $2 \times 2$ grid the shape of $L(G)$ looks like the letter $\mathrm{L}$.

Theorem 2.19 (=[I, 3.7]). When $(r, a)=1$, the elements of $I(r, a)$ are precisely those numbers in $[0, r]$ that do not appear as weights of monomials in the region $B(G) \backslash L(G)$.

Example 2.20. Consider $G=\frac{1}{17}(1,10)$. Then $B(G) \backslash L(G)$ is the region

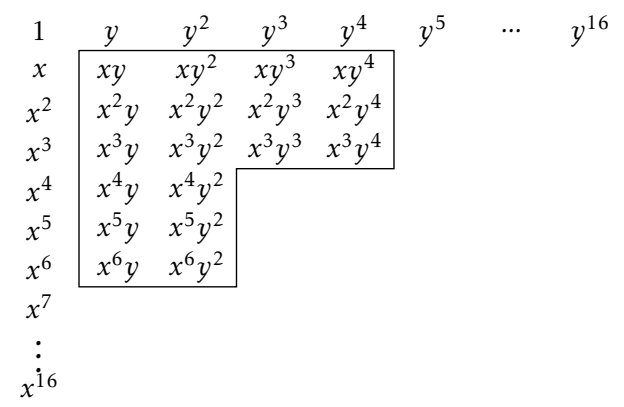

Replacing the monomials in the above region by their corresponding weights gives

\begin{tabular}{|llll|}
\hline 11 & 4 & 14 & 7 \\
12 & 5 & 15 & 8 \\
13 & 6 & 16 & 9 \\
14 & 7 & & \\
15 & 8 & & \\
16 & 9 & & \\
\cline { 1 - 2 } & & &
\end{tabular}

and so by 2.19 , the $i$-series consists of those numbers that do not appear in the above region, which are precisely the numbers $0,1,2,3,10$ and 17 . Indeed, in this example $\frac{17}{10}=[2,4,2,2]$ and the $i$-series is

$$
i_{0}=17>i_{1}=10>i_{2}=3>i_{3}=2>i_{4}=1>i_{5}=0 .
$$


The following lemma, which we use later, is an extension of 2.19. For integers $r>0$ and $k$, write $[k]_{r}$ for the unique integer $k^{\prime}$ satisfying $0 \leq k^{\prime} \leq r-1$ and $k-k^{\prime} \in r \mathbb{Z}$.

Lemma 2.21. Assume $(r, a)=1$. For $0 \leq u \leq r-1$, the following are equivalent.

(1) $u \in I(r, r-a)$.

(2) $u$ does not appear in $B(G) \backslash L(G)$ for $G:=\frac{1}{r}(1,-a)$.

(3) For every integer $\ell \geq 1$, there exists an integer $m \in[1, \ell]$ such that $[u+\ell a-1]_{r} \geq[m a-1]_{r}$.

Proof. (1) $\Leftrightarrow(2)$ This is 2.19.

$(2) \Leftrightarrow(3)$ We will establish the following claim: $u$ does not appear in column $\ell$ of $B(G) \backslash L(G)$ if and only if there exists an integer $m$ satisfying $1 \leq m \leq \ell$ and $[u+\ell a-1]_{r} \geq[m a-1]_{r}$.

The first row of $B(G)$ is $0,-a,-2 a,-3 a, \ldots$. Now for each $m$ with $1 \leq m \leq \ell$, we find the first occurrence of weight 0 in column $m$, and use this to draw the following diagram:

\begin{tabular}{|cc:c}
$-m a$ & $\cdots$ & $-\ell a$ \\
\hline $1-m a$ & $\cdots$ & $1-\ell a$ \\
$2-m a$ & $\cdots$ & $2-\ell a$ \\
$\vdots$ & $\ddots$ & $\vdots$ \\
-2 & $\cdots$ & $-2+(m-\ell) a$ \\
-1 & $\cdots$ & $-1+(m-\ell) a$ \\
\hline 0 & $\cdots$ & $(m-\ell) a$
\end{tabular}

The column $\ell$ of $B(G) \backslash L(G)$ is the intersection, over all $m$ with $1 \leq m \leq \ell$, of the above dotted regions. It is clear that $u$ does not appear in the dotted region in the above diagram if and only if $[u+\ell a-1]_{r} \geq[m a-1]_{r}$. The claim follows.

Notation 2.22. Throughout the remainder of the paper, to aid readability we will use the following simplified notation.

\begin{tabular}{ccc}
\hline Notation & Meaning & Simplified Notation \\
\hline $\mathbb{X}_{\mathbf{p}, \lambda}$ & Weighted projective line & $\mathbb{X}$ \\
$S_{\mathbf{p}, \lambda}$ & Defining ring of $\mathbb{X}_{\mathbf{p}, \lambda}$ & $S$ \\
$\Lambda_{\mathbf{p}, \lambda}$ & Canonical algebra & $\Lambda$ \\
$S_{\mathbf{p}, \lambda}^{\vec{x}}$ & Veronese of $S_{\mathbf{p}, \lambda}$ with respect to $\vec{x} \in \mathbb{L}$ & $S^{\vec{x}}$ \\
$Y_{\mathbf{p}, \lambda}^{\overrightarrow{\mathbf{x}}}$ & Resolution of Spec $S_{\mathbf{p}, \lambda}^{\vec{x}}$ in (1.C) & $Y^{\vec{x}}$ \\
\hline
\end{tabular}

Throughout it will be implicit that we are working generally, with parameters $(\mathbf{p}, \lambda)$.

\section{The Total Space $\mathbb{T}$}

\subsection{Definition and First Properties}

With notation as in 2.22 , let $\vec{x} \in \mathbb{L}$ and consider the Veronese subring $S^{\vec{x}}$, and the total space stack defined by

$$
\mathbb{T}^{\vec{x}}:=\operatorname{Tot}\left(\mathcal{O}_{X}(-\vec{x})\right):=[(\operatorname{Spec} S \backslash 0 \times \operatorname{Spec} \mathbb{k}[t]) / \operatorname{Spec} \mathbb{k} \mathbb{L}],
$$

where $\mathbb{L}$ acts on $t$ with weight $-\vec{x}$. There is a natural projection $q: \mathbb{T}^{\vec{x}} \rightarrow \mathbb{X}$, and a natural map $g: \mathbb{T}^{\vec{x}} \rightarrow T^{\vec{x}}$ to its coarse moduli space. 
We remark that $T^{\vec{x}}$ has a natural open cover. Indeed, the open covering Spec $S \backslash 0=U_{0} \cup U_{1}$ with $U_{i}:=\operatorname{Spec} S_{t_{i}}$ induces an open cover

$$
(\operatorname{Spec} S \backslash 0) \times \operatorname{Spec} \mathbb{k}[t]=U_{0}^{\prime} \cup U_{1}^{\prime} \quad \text { with } \quad U_{i}^{\prime}:=U_{i} \times \operatorname{Spec} \mathbb{k}[t]=\operatorname{Spec} S_{t_{i}}[t],
$$

which in turn implies that $T^{\vec{x}}$ has an open cover

$$
T^{\vec{x}}=V_{0} \cup V_{1} \text { with } V_{i}:=\operatorname{Spec}\left(S_{t_{i}}[t]\right)_{0},
$$

where $\left(S_{t_{i}}[t]\right)_{0}$ is the degree zero part of the $\mathbb{L}$-graded ring $S_{t_{i}}[t]$ with $\operatorname{deg} t=-\vec{x}$. As in 2.1(2), the curve $X_{i}:=\operatorname{Spec}\left(S_{t_{i}}\right)_{0}$ in $V_{i}$ gives the coarse moduli $X=X_{0} \cup X_{1} \cong \mathbb{P}^{1}$ of $\mathbb{X}$.

We first investigate the singularities of $T^{\vec{x}}$.

Proposition 3.1. If $\vec{x} \in \mathbb{L}$, then $T^{\vec{x}}$ is a surface containing the coarse moduli $X \cong \mathbb{P}^{1}$ of $\mathrm{X}$. Moreover $T^{\vec{x}}$ is normal, and all its singularities are isolated and lie on $X$.

Proof. Fix $i=0,1$ and let $A:=S_{t_{i}}[t]$ and $B:=\left(S_{t_{i}}[t]\right)_{0}$ so that $V_{i}=\operatorname{Spec} B$.

Since $A$ is an $\mathbb{L}$-factorial $\mathbb{L}$-domain by 2.2(1)(4), its degree zero part $B$ is a normal domain by 2.2(2). Now we claim $\operatorname{dim} B=2$. Note that $S$ is a finitely generated $\mathbb{L}$-graded module over the $\mathbb{Z} \vec{c}$-graded subring $C:=\mathbb{k}\left[t_{0}, t_{1}\right]$. Thus $A$ is a finitely generated $\mathbb{L}$-graded $C_{t_{i}}[t]$-module, and similarly $B$ is a finitely generated $\left(C_{t_{i}}[t]\right)_{0}$-module. Let $p$ be the smallest positive integer satisfying $p \vec{x} \in \mathbb{Z} \vec{c}$, and $p \vec{x}=q \vec{c}$ for $q \in \mathbb{Z}$. Then $\left(C_{t_{i}}[t]\right)_{0}=\left(C_{t_{i}}\left[t^{p}\right]\right)_{0}$ is the polynomial ring with two variables $t_{1-i} / t_{i}$ and $t_{i}^{q} t^{p}$. Thus $\operatorname{dim} B=\operatorname{dim}\left(C_{t_{i}}[t]\right)_{0}=2$.

Consider next the $\mathbb{Z}$-grading on $A=S_{t_{i}}[t]$ defined by $\operatorname{deg} t=1$ and $\operatorname{deg} x=0$ for any $x \in S_{t_{i}}$. This gives a $\mathbb{Z}$-grading on $B$ such that $B=\bigoplus_{j \geq 0} B_{j}$ and $B_{0}=\left(S_{t_{i}}\right)_{0}$. Since $B$ is a $\mathbb{Z}$-graded finitely generated $\mathbb{k}$-algebra, by the Jacobian criterion, there is a $\mathbb{Z}$-graded ideal $I$ of $B$ such that $\operatorname{Sing} B=\operatorname{Spec}(B / I)$. Since $B$ is normal, all the singularities of $B$ are isolated and $\operatorname{dim}_{\mathbb{k}}(B / I)<\infty$ holds. Since $I$ is $\mathbb{Z}$-graded, it contains $\bigoplus_{j>\ell} B_{j}$ for $\ell \gg 0$ and hence $\sqrt{I}$ contains $\bigoplus_{j>0} B_{j}$. Consequently, Sing $B$ is contained in Spec $B_{0}=X_{i} \subset X$.

Proposition 3.2. Suppose that $\vec{x} \in \mathbb{L}$ and write $\vec{x}$ in normal form as $\vec{x}=\sum_{i=1}^{n} a_{i} \vec{x}_{i}+a \vec{c}$ for some $0 \leq a_{i}<p_{i}$ and $a \in \mathbb{Z}$. Then on $X \cong \mathbb{P}^{1}$, complete locally the singularities of $T^{\vec{x}}$ are of the form

$$
\begin{array}{ccccc}
\lambda_{1} & \lambda_{2} & \lambda_{3} & \mathbb{P}^{1} & \lambda_{n} \\
\frac{1}{p_{1}} \stackrel{\bullet}{\left(1,-a_{1}\right)} & \frac{1}{p_{2}}\left(\stackrel{\bullet}{\bullet}-a_{2}\right) & \frac{1}{p_{3}}\left(1,-a_{3}\right) & \cdots & \frac{1}{p_{n}}\left(1,-a_{n}\right)
\end{array}
$$

Proof. We will show that $\widehat{\mathcal{O}}_{T^{\vec{x}}, \lambda_{i}}$ is the completion of $\frac{1}{p_{i}}\left(1,-a_{i}\right)$. By symmetry, we only have to consider the case $i=1$. We use the presentation of $S$ given in 2.1(2)

$$
S=\frac{\mathbb{k}\left[x_{1}, \ldots, x_{n}\right]}{\left(x_{i}^{p_{i}}+x_{1}^{p_{1}}-\lambda_{i} x_{2}^{p_{2}} \mid 3 \leq i \leq n\right)} .
$$

and the open cover $T^{\vec{x}}=V_{0} \cup V_{1}$ given in (3.A), where $t_{0}=x_{2}^{p_{2}}$ and $t_{1}=x_{1}^{p_{1}}$. Thus $\lambda_{1}=(1: 0)$ belongs to $V_{0}=\operatorname{Spec} B$ for $B:=\left(S_{t_{0}}[t]\right)_{0}=\left(S_{x_{2}}[t]\right)_{0}$.

Let $m$ be the maximal ideal of $B$ corresponding to $\lambda_{1}$. We shall show that $\widehat{\mathcal{O}}_{T^{\vec{x}}, \lambda_{1}}=\widehat{B}_{\mathrm{m}}$ is the completion of $\frac{1}{p_{1}}\left(1,-a_{1}\right)$. For the polynomial ring $\mathbb{k}\left[x_{1}, \ldots, x_{n}, t\right]$ and the formal power series ring $\mathbb{k}\left[\left[\mathrm{x}_{1}, \mathrm{t}\right]\right]$, consider the morphism

$$
f: \mathbb{k}\left[x_{1}, \ldots, x_{n}, t\right] \rightarrow P:=\mathbb{k}\left[\left[\mathrm{x}_{1}, \mathrm{t}\right]\right]
$$

of $\mathbb{k}$-algebras defined by $f(t)=\mathrm{t}, f\left(x_{1}\right)=\mathrm{x}_{1}, f\left(x_{2}\right)=1$ and $f\left(x_{i}\right)=\left(\lambda_{i}-\mathrm{x}_{1}^{p_{1}}\right)^{1 / p_{i}}$ for $3 \leq i \leq n$, where a $p_{i}$-th root of $\lambda_{i}-x_{1}^{p_{1}}$ exists since $\mathbb{k}$ is an algebraically closed field of characteristic zero. Since $f$ sends 
$x_{i}^{p_{i}}+x_{1}^{p_{1}}-\lambda_{i} x_{2}^{p_{2}}$ to zero for all $3 \leq i \leq n$, it induces a morphism of $\mathbb{k}$-algebras $f: S[t] \rightarrow P$, and further since $f\left(t_{0}\right)=f\left(x_{2}^{p_{2}}\right)=1$ this induces a morphism of $\mathbb{k}$-algebras

$$
f: S_{t_{0}}[t] \rightarrow P
$$

Let $C:=\frac{1}{p_{1}}\left(1,-a_{1}\right)=\langle\zeta\rangle$ be the cyclic group acting on $P$ by $\zeta \mathrm{x}_{1}=\varepsilon \mathrm{x}_{1}$ and $\zeta \mathrm{t}=\varepsilon^{-a_{1}} \mathrm{t}$ for a primitive $p_{1}$-th root $\varepsilon$ of unity. Certainly $f\left(x_{i}\right)$ with $2 \leq i \leq n$ belongs to $\mathbb{k}\left[\left[\mathrm{x}_{1}^{p_{1}}\right]\right] \subset P^{C}$. Now we claim that (3.C) induces a morphism of $\mathbf{k}$-algebras

$$
f: B=\left(S_{t_{0}}[t]\right)_{0} \rightarrow P^{C} .
$$

If a monomial $X=x_{1}^{\ell_{1}} \ldots x_{n}^{\ell_{n}} t^{\ell} \in S_{x_{2}}[t]=S_{t_{0}}[t]$ has degree zero, then $\ell_{1} \vec{x}_{1}+\ldots+\ell_{n} \vec{x}_{n}+\ell \vec{x}=0$ holds. Looking at the coefficient of $\vec{x}_{1}$, we have $\ell_{1}+\ell a_{1} \in p_{1} \mathbb{Z}$. Thus $f(X)=x_{1}^{\ell_{1}} \mathrm{t}^{\ell} \prod_{i=2}^{n} f\left(x_{i}\right)^{\ell_{i}}$ belongs to $P^{C}$, and the assertion follows.

The closed subscheme $X_{0}=\operatorname{Spec}\left(S_{t_{0}}\right)_{0}$ of $V_{0}=\operatorname{Spec} B$ is defined by the ideal $\left(t S_{t_{0}}[t]\right)_{0}$ of $B$, and the closed point $\lambda_{1}$ of $X_{0}$ is defined by the ideal $\left(t_{1} / t_{0}\right)$ of $\left(S_{t_{0}}\right)_{0}=\mathbb{k}\left[t_{1} / t_{0}\right]$. Therefore the maximal ideal $\mathrm{m}$ of $B$ is generated by monomials $x_{1}^{\ell_{1}} \cdots x_{n}^{\ell_{n}} t^{\ell}$ with $\ell \geq 1$ and $t_{1} / t_{0}=x_{1}^{p_{1}} / x_{2}^{p_{2}}$. In particular, $f(\mathrm{~m})$ is contained in the maximal ideal $n$ of $P^{C}$, and hence (3.D) induces a morphism

$$
f: \widehat{B}_{\mathrm{m}} \rightarrow P^{C} \text {. }
$$

We show that this is an isomorphism. Since $B$ is a normal domain which is finitely generated over a field, $\widehat{B}_{\mathrm{m}}$ is also a normal domain by Zariski's Main Theorem [ZS, VIII.13 Theorem 32]. Since $\operatorname{dim} \widehat{B}_{\mathfrak{m}}=2=\operatorname{dim} P^{C}$, it suffices to show that (3.E) is surjective, or equivalently, (3.D) gives a surjective map $m \rightarrow n / n^{2}$. Since the $\mathbb{k}$-vector space $\mathfrak{n} / \mathfrak{n}^{2}$ is spanned by monomials in $P^{C}$, it suffices to show that any monomial $x_{1}^{\ell_{1}} t^{\ell}$ in $P^{C}$ belongs to $\operatorname{Im} f+n^{2}$. Since $x_{1}^{\ell_{1}} \mathrm{t}^{\ell}$ is invariant under the action of $C$, the coefficient of $\vec{x}_{1}$ in the normal form of $\ell_{1} \vec{x}_{1}+\ell \vec{x}$ is zero. Thus there exist $\ell_{2} \in \mathbb{Z}$ and $\ell_{3}, \ldots, \ell_{n} \in \mathbb{Z}_{\geq 0}$ such that $\ell_{1} \vec{x}_{1}+\ldots+\ell_{n} \vec{x}_{n}+\ell \vec{x}=0$. Now $X:=x_{1}^{\ell_{1}} \cdots x_{n}^{\ell_{n}} t^{\ell} \in B$ satisfies

$$
f(\alpha X) \equiv \mathrm{x}_{1}^{\ell_{1}} \mathrm{t}^{\ell} \operatorname{modn}{ }^{2} \text { for } \alpha:=\prod_{i=3}^{n} \lambda_{i}^{-\ell_{i} / p_{i}} .
$$

Hence (3.E) is an isomorphism.

The following calculation will be one of our main technical tools.

Proposition 3.3. For any $\vec{x} \in \mathbb{L}$,

$$
H^{i}\left(\mathcal{O}_{T^{\vec{x}}}\right)= \begin{cases}\bigoplus_{j \geq 0} S_{j \vec{x}} & i=0, \\ \bigoplus_{j \geq 0}\left(S_{\vec{\omega}-j \vec{x}}\right)^{*} & i=1, \\ 0 & i \geq 2\end{cases}
$$

Therefore there is a canonical morphism $\gamma: T^{\vec{x}} \rightarrow \operatorname{Spec} S^{\mathbb{N} \vec{x}}$.

Proof. We calculate $H^{i}\left(\mathcal{O}_{T^{\vec{x}}}\right)$ as the Čech cohomology with respect to the open affine cover $T^{\vec{x}}=V_{0} \cup V_{1}$ in (3.A). Since $H^{0}\left(V_{i}, \mathcal{O}_{T^{\vec{x}}}\right)=\left(S_{t_{i}}[t]\right)_{0}$ for $i=0,1$ and $H^{0}\left(V_{0} \cap V_{1}, \mathcal{O}_{T^{\vec{x}}}\right)=\left(S_{t_{0} t_{1}}[t]\right)_{0}$, the complex

$$
0 \rightarrow\left(S_{t_{0}}[t]\right)_{0} \oplus\left(S_{t_{1}}[t]\right)_{0} \rightarrow\left(S_{t_{0} t_{1}}[t]\right)_{0} \rightarrow 0
$$

has cohomology $H^{i}\left(\mathcal{O}_{T^{\vec{x}}}\right)$ with $i \geq 0$. Thus $H^{i}\left(\mathcal{O}_{T^{\vec{x}}}\right)=0$ for any $i \geq 2$.

Now let $\mathfrak{a}=S t_{0}+S t_{1}$. Then the local cohomologies $H_{\mathfrak{a}}^{i}(S)$ of $S$ are the cohomologies of the extended Čech complex

$$
0 \rightarrow S \rightarrow S_{t_{0}} \oplus S_{t_{1}} \rightarrow S_{t_{0} t_{1}} \rightarrow 0
$$


by [BS, Theorem 5.1.20]. Since $t_{0}, t_{1}$ is an $S$-sequence, we have $H_{\mathfrak{a}}^{0}(S)=H_{\mathfrak{a}}^{1}(S)=0$ by [BS, Theorem 6.2.7]. Thus (3.G) gives an exact sequence

$$
0 \rightarrow(S[t])_{0} \rightarrow\left(S_{t_{0}}[t]\right)_{0} \oplus\left(S_{t_{1}}[t]\right)_{0} \rightarrow\left(S_{t_{0} t_{1}}[t]\right)_{0} \rightarrow\left(H_{\mathfrak{a}}^{2}(S) \otimes_{\mathbb{k}} \mathbb{k}[t]\right)_{0} \rightarrow 0 .
$$

Comparing with (3.F) gives isomorphisms

$$
H^{0}\left(\mathcal{O}_{T^{\vec{x}}}\right) \simeq(S[t])_{0}=\bigoplus_{j \geq 0} S_{j \vec{x}} \text { and } H^{1}\left(\mathcal{O}_{T^{\vec{x}}}\right) \simeq\left(H_{\mathfrak{a}}^{2}(S) \otimes_{\mathbb{k}} \mathbb{k}[t]\right)_{0}=\bigoplus_{j \geq 0} H_{\mathfrak{a}}^{2}(S)_{j \vec{x}}
$$

Since $\sqrt{\mathfrak{a}}$ is the $\mathbb{L}$-graded maximal ideal $\mathfrak{m}$ of $S$, we have $H_{\mathfrak{a}}^{i}(S)=H_{\mathfrak{m}}^{i}(S)$. Furthermore, $S(\vec{\omega})$ being an $\mathbb{L}$-graded canonical module of $S$, the $\mathbb{L}$-graded local duality theorem [BS, Theorem 14.4.1] gives the required isomorphism

$$
H_{\mathfrak{a}}^{2}(S)_{j \vec{x}}=H_{\mathfrak{m}}^{2}(S)_{j \vec{x}} \simeq\left(\operatorname{Hom}_{S}(S, S(\vec{\omega}))_{-j \vec{x}}\right)^{*}=\left(S_{\vec{\omega}-j \vec{x}}\right)^{*} .
$$

The last statement follows from [H1, Exercise II.2.4], since $S^{\mathbb{N} \vec{x}}:=\bigoplus_{j \geq 0} S_{j \vec{x}}$.

There is also a map $f: \mathbb{X} \rightarrow X$ from $\mathbb{X}$ to its coarse moduli space, and $p: T^{\vec{x}} \rightarrow X$ an obvious morphism, which together with the above form a commutative diagram

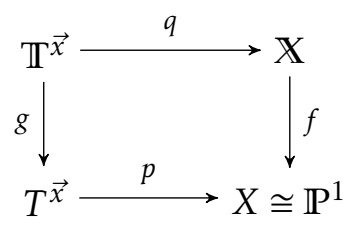

We now try to contract the $\mathbb{P}^{1}$ in (3.B) by taking global sections. As is usual, to do this requires some form of negativity for $\mathbb{T}^{\vec{x}}=\mathbb{T o t}\left(\mathcal{O}_{\mathbb{X}}(-\vec{x})\right)$; in the language here, this translates into some form of positivity for $\vec{x}$. This is slightly technical to state, and we will require the following lemma. Recall that there is a group homomorphism

$$
\delta: \mathbb{L} \rightarrow \mathbb{Q}
$$

sending $\vec{c} \mapsto 1$ and $\vec{x}_{i} \mapsto \frac{1}{p_{i}}$. It is elementary that $\delta\left(\mathbb{L}_{+}\right) \subset \mathbb{Q}_{\geq 0}$, and $\vec{x}$ is torsion if and only if $\delta(\vec{x})=0$. Also, using normal form, it is clear that $\mathbb{L} \backslash \mathbb{L}_{+}$has the maximum element $\sum_{i=1}^{n}\left(p_{i}-1\right) \vec{x}_{i}-\vec{c}=\vec{\omega}+\vec{c}$. In particular, $\vec{\omega} \notin \mathbb{L}_{+}$.

Lemma 3.4. If $\vec{x} \in \mathbb{L}$, then the following hold.

(1) $-i \vec{x} \notin \mathbb{L}_{+}$for all $i>0 \Longleftrightarrow \delta(\vec{x})>0$.

(2) $\vec{\omega}-i \vec{x} \notin \mathbb{L}_{+}$for all $i \geq 0 \Rightarrow \delta(\vec{x}) \geq 0$.

Proof. $(1)(\Leftarrow)$ If $i>0$, then $\delta(-i \vec{x})=-i \delta(\vec{x})<0$. The result follows since $\delta\left(\mathbb{L}_{+}\right) \subset \mathbb{Q}_{\geq 0}$. $(1)(2)(\Rightarrow)$ Since $\vec{\omega}+\vec{c}$ is the maximum element of $\mathbb{L} \backslash \mathbb{L}_{+}$, any $\vec{z} \in \mathbb{L}$ satisfying

$$
\delta(\vec{z})>\delta(\vec{\omega}+\vec{c})
$$

belongs to $\mathbb{L}_{+}$. If $\delta(\vec{x})<0$, then

$$
\delta(-j \vec{x})=-j \delta(\vec{x}) \rightarrow+\infty \text { and } \delta(\vec{\omega}-j \vec{x})=\delta(\vec{\omega})-j \delta(\vec{x}) \rightarrow+\infty
$$

as $j \rightarrow \infty$. Hence for sufficiently large $j$, both $\delta(-j \vec{x})$ and $\delta(\vec{\omega}-j \vec{x})$ are larger than $\delta(\vec{\omega}+\vec{c})$. Thus, by (3.I), both $-j \vec{x}$ and $\vec{\omega}-j \vec{x}$ belong to $\mathbb{L}_{+}$, a contradiction. Hence $\delta(\vec{x}) \geq 0$. Thus (2) follows. To complete the proof of $(1)(\Rightarrow)$, notice that the assumption implies that $\vec{x}$ is not torsion, as in the proof of 2.3(3), so $\delta(\vec{x}) \neq 0$. 
This leads to our key new definition.

Definition 3.5. We define the geometrically positive elements of $\mathbb{L}$ to be

$$
\operatorname{GPos}(\mathbb{L}):=\left\{\vec{x} \in \mathbb{L} \mid \vec{x} \text { is not torsion, and } \vec{\omega}-j \vec{x} \notin \mathbb{L}_{+} \text {for all } j \geq 0\right\} .
$$

Given any $\vec{x} \in \mathbb{L}$, recall from 3.3 that $H^{0}\left(\mathcal{O}_{T^{\vec{x}}}\right)=S^{\mathbb{N} \vec{x}}$ holds, giving rise to a canonical morphism $\gamma: T^{\vec{x}} \rightarrow \operatorname{Spec} S^{\mathbb{N} \vec{x}}$.

Proposition 3.6. Suppose that $\vec{x} \in \mathbb{L}$.

(1) If $0 \neq \vec{x} \in \mathbb{L}_{+}$, then $\vec{x} \in \operatorname{GPos}(\mathbb{L})$.

(2) The following conditions are equivalent.

(a) $\vec{x} \in \operatorname{GPos}(\mathbb{L})$.

(b) $-i \vec{x} \notin \mathbb{L}_{+}$for all $i>0$, and $\vec{\omega}-j \vec{x} \notin \mathbb{L}_{+}$for all $j \geq 0$.

(c) $S^{\mathbb{N} \vec{x}}=S^{\vec{x}}$ and $\mathbf{R}^{t} \gamma_{*} \mathcal{O}_{T^{\vec{x}}}=0$ for all $t>0$.

Proof. (1) Clearly $\vec{x}$ is not torsion. Since $\vec{\omega} \notin \mathbb{L}_{+}$and $\vec{\omega}-j \vec{x} \leq \vec{\omega}$ for $j \geq 0$, we have $\vec{\omega}-j \vec{x} \notin \mathbb{L}_{+}$. Thus $\vec{x} \in \operatorname{GPos}(\mathbb{L})$.

(2)(a) $\Leftrightarrow(\mathrm{b})$. The condition $\vec{\omega}-j \vec{x} \notin \mathbb{L}_{+}$for all $j \geq 0$ is common to both. Thus we just need to prove, assuming this condition, that $\vec{x}$ is not torsion (equivalently, $\delta(\vec{x}) \neq 0$ ) if and only if $-i \vec{x} \notin \mathbb{L}_{+}$for all $i>0$. But this follows from 3.4.

$(\mathrm{b}) \Leftrightarrow(\mathrm{c})$ Follows from 3.3.

Corollary 3.7. If $\vec{x} \in \mathrm{GPos}(\mathbb{L})$, then there is a canonical morphism

$$
\gamma: T^{\vec{x}} \rightarrow \operatorname{Spec} S^{\vec{x}}
$$

such that $\mathbf{R} \gamma_{*} \mathcal{O}_{T^{\vec{x}}}=\mathcal{O}_{S^{\vec{x}}}$.

\subsection{The morphism $\gamma$}

In this subsection we show, under the assumption in 3.7, that $\gamma$ is a projective birational morphism. This then implies that $T^{\vec{x}}$ is a partial resolution of singularities of Spec $S^{\vec{x}}$, which indeed is our motivation for studying the stack $\mathbb{T}^{\vec{x}}$ and its coarse moduli $T^{\vec{x}}$.

Lemma 3.8. Suppose that $\vec{x} \in \mathrm{GPos}(\mathbb{L})$. Then

(1) $\gamma$ is a finite type morphism between noetherian schemes.

(2) $\mathcal{L}:=p^{*} \mathcal{O}(1)$ is an ample bundle on $T^{\vec{x}}$.

(3) $\mathcal{L}$ is $\gamma$-relatively ample.

Proof. (1) $T^{\vec{x}}$ is noetherian since it is covered by a finite number of affine charts (namely two) in (3.A), each given by a noetherian ring. Further $\operatorname{Spec} S^{\vec{x}}$ is noetherian since $S^{\vec{x}}$ is by 2.3. Now the morphism $\gamma$ is quasi-compact since $T^{\vec{x}}$ is noetherian and thus quasi-compact, and Spec $S^{\vec{x}}$ is affine. Further, composing $\gamma$ with the structure morphisms $s: \operatorname{Spec} S^{\vec{x}} \rightarrow$ Spec $\mathbb{k}$ gives a morphism $s \circ \gamma$ of finite type, since $T^{\vec{x}}$ is covered by finitely generated $\mathrm{k}$-algebras. By the left cancelation property [H1, II.Ex.3.13(f)], $\gamma$ also has finite type.

(2) It is well-known that $\mathcal{O}(1)$ is ample on $\mathbb{P}^{1}$, or equivalently, relatively ample with respect to the structure morphism $\mathbb{P}^{1} \rightarrow$ Spec $\mathbb{k}$. Since $p$ is affine, pulling back yields a bundle $p^{*} \mathcal{O}(1)$ which is relatively ample with respect to the composition $T^{\vec{x}} \rightarrow \mathbb{P}^{1} \rightarrow$ Spec $\mathbb{k}$ [EGA, II.5.1.12]. But this is just the structure morphism for $T^{\vec{x}}$, hence it follows that $p^{*} \mathcal{O}(1)$ is ample on $T^{\vec{x}}$.

(3) This follows immediately from (2), given $\operatorname{Spec} S^{\vec{x}}$ is affine. 
As notation, for $\vec{y} \in \mathbb{L}$ write $S(\vec{y})^{\vec{x}}:=\bigoplus_{i \in \mathbb{Z}} S_{\vec{y}+i \vec{x}} \supset S(\vec{y})^{\mathbb{N} \vec{x}}:=\bigoplus_{i \geq 0} S_{\vec{y}+i \vec{x}}$.

Lemma 3.9. Suppose that $\vec{x} \in \mathrm{GPos}(\mathbb{L})$. Then

(1) For all $\vec{y} \in \mathbb{L}, \gamma_{*} g_{*} q^{*} \mathcal{O}_{\mathbf{X}}(\vec{y})=S(\vec{y})^{\mathbb{N} \vec{x}}$.

(2) $\gamma_{*} \mathcal{L}=S(\vec{c})^{\mathbb{N} \vec{x}}$ holds, and this is a finitely generated $S^{\vec{x}}$-module.

(3) $\gamma_{*} \mathcal{L}^{-n}$ and $\mathbf{R}^{1} \gamma_{*} \mathcal{L}^{-n}$ are finitely generated $S^{\vec{x}}$-modules for all $n \geq 0$.

Proof. (1) Note first that

$$
\gamma_{*} g_{*} q^{*} \mathcal{O}_{\mathbf{X}}(\vec{y})=\mathrm{H}^{0}\left(\mathbb{T}, q^{*} \mathcal{O}_{\mathbf{X}}(\vec{y})\right)=\mathrm{H}^{0}\left(\mathbb{X}, q_{*} q^{*} \mathcal{O}_{\mathbf{X}}(\vec{y})\right) .
$$

By the projection formula $q_{*} q^{*}\left(\mathcal{O}_{\mathbf{X}}(\vec{y})\right)=\bigoplus_{i \geq 0} \mathcal{O}_{\mathbf{X}}(i \vec{x}+\vec{y})$, and so the above equals

$$
\bigoplus_{i \geq 0} \mathrm{H}^{0}\left(\mathbf{X}, \mathcal{O}_{\mathbf{X}}(i \vec{x}+\vec{y})\right)=\bigoplus_{i \geq 0} S_{i \vec{x}+\vec{y}}=S(\vec{y})^{\mathbb{N} \vec{x}}
$$

(2) Note that $g_{*} g^{*} \mathcal{L}=\mathcal{L}$ by the projection formula, and so

$$
\gamma_{*} \mathcal{L}=\gamma_{*} g_{*} g^{*} \mathcal{L}=\gamma_{*} g_{*} g^{*} p^{*} \mathcal{O}_{\mathbb{P}^{1}}(1)=\gamma_{*} g_{*} q^{*} f^{*} \mathcal{O}_{\mathbb{P}^{1}}(1)=\gamma_{*} g_{*} q^{*} \mathcal{O}_{\mathbf{X}}(\vec{c}) .
$$

Hence $\gamma_{*} \mathcal{L}=S(\vec{c})^{\mathbb{N} \vec{x}}$ by (1). Now by 2.3(1), $S(\vec{c})^{\vec{x}}$ is a finitely generated $S^{\vec{x}}$-module, hence its submodule $\gamma_{*} \mathcal{L}$ is also a finitely generated $S^{\vec{x}}$-module, since $S^{\vec{x}}$ is noetherian.

(3) We know by 3.7 that the result is true for $n=0$ since $\mathbf{R} \gamma_{*} \mathcal{O}_{T^{\vec{x}}}=\mathcal{O}_{S^{\vec{x}}}$. Part (2) shows that $f_{*} \mathcal{L}$ is finitely generated. Pulling up the Euler exact sequence from $\mathbb{P}^{1}$ gives an exact sequence

$$
0 \rightarrow \mathcal{L}^{-1} \rightarrow \mathcal{O}^{\oplus 2} \rightarrow \mathcal{L} \rightarrow 0
$$

on $T^{\vec{x}}$, and pushing down gives an exact sequence

$$
0 \rightarrow \gamma_{*} \mathcal{L}^{-1} \rightarrow\left(S^{\vec{x}}\right)^{\oplus 2} \rightarrow \gamma_{*} \mathcal{L} \rightarrow \mathbf{R}^{1} \gamma_{*} \mathcal{L}^{-1} \rightarrow 0 .
$$

Since $S^{\vec{x}}$ is noetherian, and the middle two objects are finitely generated, necessarily the outer objects are also finitely generated. Hence the result is true for $n=1$.

By induction, we can thus assume that the result is true for $n-1$ and $n-2$. Twisting the sequence (3.J) appropriately, then pushing down, gives an exact sequence

$$
0 \rightarrow \gamma_{*} \mathcal{L}^{-n} \rightarrow\left(\gamma_{*} \mathcal{L}^{-n+1}\right)^{\oplus 2} \rightarrow \gamma_{*} \mathcal{L}^{-n+2} \rightarrow \mathbf{R}^{1} \gamma_{*} \mathcal{L}^{-n} \rightarrow\left(\mathbf{R}^{1} \gamma_{*} \mathcal{L}^{-n+1}\right)^{\oplus 2} \rightarrow \mathbf{R}^{1} \gamma_{*} \mathcal{L}^{-n+2} \rightarrow 0
$$

By induction the second, third, fifth and sixth objects are finitely generated. Hence so too are the first and fourth. By induction the result follows.

Theorem 3.10. Suppose that $\vec{x} \in \operatorname{GPos}(\mathbb{L})$. Then $\gamma: T^{\vec{x}} \rightarrow \operatorname{Spec} S^{\vec{x}}$ is a projective birational morphism, satisfying $\mathbf{R} \gamma_{*} \mathcal{O}_{T^{\vec{x}}}=\mathcal{O}_{S^{\vec{x}}}$.

Proof. We first claim that $\gamma$ is proper. Since by 3.8(1) $\gamma$ is a finite type morphism between noetherian schemes, by [R3] it suffices to show that both $\gamma_{*}$ and $\mathbf{R}^{1} \gamma_{*}$ preserve coherent sheaves. Pick $\mathcal{F} \in \operatorname{coh} T^{\vec{x}}$.

Since by 3.8(2) $\mathcal{L}$ is ample, there exists some $n \geq 0$ such that $\mathcal{F} \otimes \mathcal{L}^{n}$ is generated by its global sections. Hence for some $N>0$ there exists a surjection $\mathcal{O}^{\oplus N} \rightarrow \mathcal{F} \otimes \mathcal{L}^{n}$ and thus a surjection $\left(\mathcal{L}^{-n}\right)^{\oplus N} \rightarrow \mathcal{F}$. Write $\mathcal{K}$ for the kernel, then pushing down yields an exact sequence

$$
0 \rightarrow \gamma_{*} \mathcal{K} \rightarrow \gamma_{*}\left(\mathcal{L}^{-n}\right)^{\oplus N} \rightarrow \gamma_{*} \mathcal{F} \rightarrow \mathbf{R}^{1} \gamma_{*} \mathcal{K} \rightarrow \mathbf{R}^{1} \gamma_{*}\left(\mathcal{L}^{-n}\right)^{\oplus N} \rightarrow \mathbf{R}^{1} \gamma_{*} \mathcal{F} \rightarrow 0
$$


since $\mathbf{R}^{2} \gamma_{*}=0$ by Čech cohomology. But $\mathbf{R}^{1} \gamma_{*}\left(\mathcal{L}^{-n}\right)^{\oplus N}$ is coherent by 3.9(3), so it follows from the above exact sequence that $\mathbf{R}^{1} \gamma_{*} \mathcal{F}$ is also coherent. Since $\mathcal{F}$ was an arbitrary coherent sheaf, we also deduce that $\mathbf{R}^{1} \gamma_{*} \mathcal{K}$ is coherent. Thus in the above exact sequence, combining with 3.9(3) we see that the second, fourth, fifth and sixth objects are coherent. It follows that the third one is too, namely $\gamma_{*} \mathcal{F}$.

Hence $\gamma$ is proper. Further $\mathcal{L}$ is $\gamma$-relatively ample by 3.8(3), and Spec $S^{\vec{x}}$ is separated since it is affine, and it is clearly quasi-compact. It is well known that these conditions imply that $\gamma$ is projective [EGA, II.5.5.3]. Lastly, $\gamma$ is birational by inspection, and the statement $\mathbf{R} \gamma_{*} \mathcal{O}_{T^{\vec{x}}}=\mathcal{O}_{S_{\vec{x}}}$ is just 3.7.

Corollary 3.11. Suppose that $\vec{x} \in \mathrm{GPos}(\mathbb{L})$. Then $S^{\vec{x}}$ is a rational surface singularity.

Proof. By 2.3(1), $S^{\vec{x}}$ is two-dimensional and noetherian. Further, by 3.10, $\gamma: T^{\vec{x}} \rightarrow \operatorname{Spec} S^{\vec{x}}$ is a projective birational morphism such that $\mathbf{R} \gamma_{*} \mathcal{O}_{T^{\vec{x}}}=\mathcal{O}_{S^{\vec{x}}}$. Now by 3.2, all the singularities on $T^{\vec{x}}$ are rational, hence there exists a resolution $\varphi: Y \rightarrow T^{\vec{x}}$ such that $\mathbf{R} \varphi_{*} \mathcal{O}_{Y}=\mathcal{O}_{T^{\vec{x}}}$. Composing gives a projective birational morphism

$$
\gamma \circ \varphi: Y \rightarrow \operatorname{Spec} S^{\vec{x}}
$$

such that $\mathbf{R}(\gamma \circ \varphi)_{*} \mathcal{O}_{Y}=\mathcal{O}_{S^{\vec{x}}}$.

In the sequel write $\varphi: Y^{\vec{x}} \rightarrow T^{\vec{x}}$ for the minimal resolution of $T^{\vec{x}}$, and consider the composition $\pi: Y^{\vec{x}} \rightarrow T^{\vec{x}} \rightarrow \operatorname{Spec} S^{\vec{x}}$. We remark that this composition need not be the minimal resolution of Spec $S^{\vec{x}}$, and indeed later in 3.17 we give a precise criterion for when it is. Nevertheless, as in the introduction, we summarize the above information in the following commutative diagram

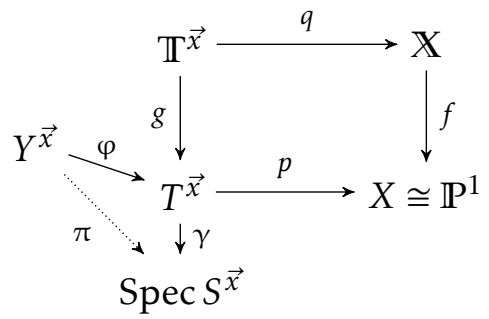

\subsection{Tilting on $\mathbb{T}^{\vec{x}}$ and $T^{\vec{x}}$}

Write $\mathcal{V}:=\mathcal{O}_{\mathbb{P}^{1}} \oplus \mathcal{O}_{\mathbb{P}^{1}}(1) \in \operatorname{coh} \mathbb{P}^{1}$, and $\mathcal{E}:=\bigoplus_{\vec{y} \in[0, \vec{c}]} \mathcal{O}_{\mathbb{X}}(\vec{y}) \in \operatorname{coh} X$. The following result is well known.

Theorem 3.12. The following statements hold.

(1) $\mathcal{V}$ is a tilting bundle on $\mathbb{P}^{1}$.

(2) $\mathcal{E}$ is a tilting bundle on $\mathrm{X}$.

Proof. Part (1) is [B1] and part (2) is [GL1].

In this subsection we lift these tilting bundles to tilting bundles on both $T^{\vec{x}}$ and $\mathbb{T}^{\vec{x}}$, again under the assumption that $0 \neq \vec{x} \in \mathbb{L}_{+}$. This is the singular line bundle (respectively stack) version of the usual trick of lifting tilting bundles on projective Fano varieties to the total spaces of various vector bundles, considered by many authors [AU, B2, BH, VdB2]. We remark that without the restriction to $\mathbb{L}_{+}$, the following is false.

Theorem 3.13. If $0 \neq \vec{x} \in \mathbb{L}_{+}$, then $q^{*} \mathcal{E}$ is a tilting bundle on $\mathbb{T}^{\vec{x}}$ such that

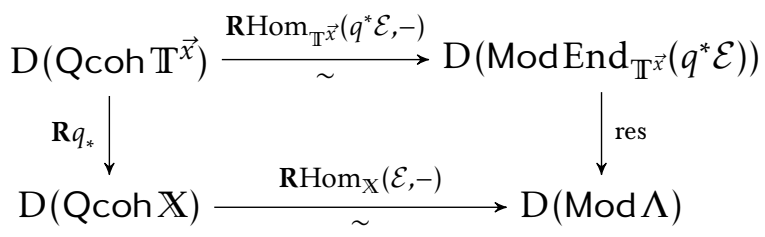


commutes, where $\Lambda$ is the canonical algebra.

Proof. To simplify, we drop all $\vec{x}$ from the notation and set $\mathbb{T}:=\mathbb{T}^{\vec{x}}$. The generation argument is standard, as in [AU, 4.1] and [B2, 4.1], namely if $M \in \mathrm{D}(\mathrm{Q} \operatorname{coh} \mathbb{T})$ with $\operatorname{Hom}_{\mathrm{D}(\mathbb{T})}\left(q^{*} \mathcal{E}, M[i]\right)=0$ for all $i$, then $\operatorname{Hom}_{\mathrm{D}(\mathrm{X})}\left(\mathcal{E}, q_{*} M[i]\right)=0$ for all $i$, so since $\mathcal{E}$ generates $\mathrm{D}(\mathrm{Q}$ coh $\mathrm{X}), q_{*} M=0$ and so since $q$ is affine $M=0$. Hence $q^{*} \mathcal{E}$ generates $\mathrm{D}(\mathrm{Q}$ coh $\mathbb{T})$.

For Ext vanishing,

$$
\begin{aligned}
\operatorname{Ext}_{\mathbb{T}}^{1}\left(q^{*} \mathcal{E}, q^{*} \mathcal{E}\right) & \cong \operatorname{Ext}_{\mathbf{X}}^{1}\left(\mathcal{E}, q_{*} q^{*} \mathcal{E}\right) \\
& \cong \operatorname{Ext}_{\mathbf{X}}^{1}\left(\mathcal{E}, \bigoplus_{k \geq 0} \mathcal{E} \otimes \mathcal{O}_{\mathbf{X}}(k \vec{x})\right) \\
& \cong \bigoplus_{k \geq 0} \operatorname{Ext}_{\mathbf{X}}^{1}\left(\mathcal{E}, \mathcal{E} \otimes \mathcal{O}_{\mathbf{X}}(k \vec{x})\right) \\
& \cong \bigoplus_{k \geq 0} \bigoplus_{i \in[0, \vec{c}]} \bigoplus_{j \in[0, \vec{c}]} H^{1}\left(\mathbf{X}, \mathcal{O}_{\mathbf{X}}(i-j+k \vec{x})\right) \\
& \cong \bigoplus_{k \geq 0} \bigoplus_{i \in[0, \vec{c}]} \bigoplus_{j \in[0, \vec{c}]} H^{0}\left(\mathbb{X}, \mathcal{O}_{\mathbf{X}}(\vec{\omega}-i+j-k \vec{x})\right)^{*}
\end{aligned}
$$

(by adjunction)

(by projection formula)

(by Serre duality)

It suffices to check that $\vec{\omega}-i+j-k \vec{x} \notin \mathbb{L}_{+}$for all $k \geq 0$ and all $i, j \in[0, \vec{c}]$. Since $0 \neq \vec{x} \in \mathbb{L}_{+}$, clearly if suffices to check $i=k=0$ and $j=\vec{c}$, this being the most positive case. But $\vec{\omega}=(n-2) \vec{c}-\sum_{t=1}^{n} \vec{x}_{t}$, and so $\vec{\omega}+\vec{c}=(n-1) \vec{c}-\sum_{t=1}^{n} \vec{x}_{t} \notin \mathbb{L}_{+}$, as required. Replacing $\operatorname{Ext}^{1}$ by Ext ${ }^{i}$, the above proof also shows that the higher Exts vanish.

The commutativity is just the adjunction $\operatorname{RHom}_{\mathbb{X}}\left(\mathcal{E}, \mathbf{R} q_{*}(-)\right) \cong \operatorname{RHom}_{\mathbb{T}}\left(q^{*} \mathcal{E},-\right)$.

Theorem 3.14. If $0 \neq \vec{x} \in \mathbb{L}_{+}$, then $p^{*} \mathcal{V}$ is a tilting bundle on $T^{\vec{x}}$.

Proof. As above, when possible we drop all $\vec{x}$ from the notation. The generation argument is identical to the argument in 3.13. The Ext-vanishing is also similar, namely writing $\mathcal{F}:=f^{*} \mathcal{V}=\mathcal{O}_{\mathbf{X}} \oplus \mathcal{O}_{\mathbf{X}}(\vec{c})$ then there is a chain of isomorphisms

$$
\begin{aligned}
\operatorname{Ext}_{T}^{i}\left(p^{*} \mathcal{V}, p^{*} \mathcal{V}\right) & \cong \operatorname{Ext}_{T}^{i}\left(p^{*} \mathcal{V}, g_{*} \mathcal{O}_{\mathbb{T}} \otimes_{T} p^{*} \mathcal{V}\right) \\
& \cong \operatorname{Ext}_{T}^{i}\left(p^{*} \mathcal{V}, g_{*} g^{*} p^{*} \mathcal{V}\right) \\
& \cong \operatorname{Ext}_{\mathbb{T}}^{i}\left(g^{*} p^{*} \mathcal{V}, g^{*} p^{*} \mathcal{V}\right) \\
& \cong \operatorname{Ext}_{\mathbb{T}}^{i}\left(q^{*} f^{*} \mathcal{V}, q^{*} f^{*} \mathcal{V}\right) \\
& \cong \operatorname{Ext}_{\mathbb{T}}^{i}\left(q^{*} \mathcal{F}, q^{*} \mathcal{F}\right)
\end{aligned}
$$

(projection formula)

(adjunction)

(commutativity of (3.K))

which is zero by 3.13 since $q^{*} \mathcal{F}$ is a summand of $q^{*} \mathcal{E}$.

Since $\pi: Y^{\vec{x}} \rightarrow \operatorname{Spec} S^{\vec{x}}$ is a resolution of a rational surface singularity, the fundamental cycle exists.

Corollary 3.15. If $0 \neq \vec{x} \in \mathbb{L}_{+}$, then the fundamental cycle associated to the morphism $\pi: Y^{\vec{x}} \rightarrow \operatorname{Spec} S^{\vec{x}}$ is reduced.

Proof. Resolving the singularities in (3.B) it is clear that the dual graph of $\pi$ is star shaped, with the middle curve of this star corresponding to the $\mathbb{P}^{1}$ in $T^{\vec{x}}$. By 3.14 the line bundle $\mathcal{L}:=p^{*} \mathcal{O}_{\mathbb{P}^{1}}(1)$ on $T^{\vec{x}}$ satisfies $\operatorname{Ext}_{T^{\vec{x}}}^{1}\left(\mathcal{L}, \mathcal{O}_{T^{\vec{x}}}\right)=0$. It clearly has degree one on the exceptional curve. Then $\mathcal{L}_{Y}:=\varphi^{*} \mathcal{L}=\mathbf{L} \varphi^{*} \mathcal{L}$ is a line bundle on $Y^{\vec{x}}$, with degree one on the middle curve and degree zero on all other curves. Furthermore

$$
H^{1}\left(\mathcal{L}_{Y}^{-1}\right) \cong \operatorname{Ext}_{Y \vec{x}}^{1}\left(\mathcal{L}_{Y}, \mathcal{O}_{Y^{\vec{x}}}\right)
$$




$$
\begin{aligned}
& \cong \operatorname{Hom}_{D^{\mathrm{b}}\left(Y^{\vec{x}}\right)}\left(\mathbf{L} \varphi^{*} \mathcal{L}, \mathcal{O}_{Y^{\vec{x}}}[1]\right) \\
& \cong \operatorname{Hom}_{D^{\mathrm{b}}\left(T^{\vec{x}}\right)}\left(\mathcal{L}, \mathbf{R} \varphi_{*} \mathcal{O}_{Y^{\vec{x}}}[1]\right) \\
& \cong \operatorname{Ext}_{T^{\vec{x}}}^{1}\left(\mathcal{L}, \mathcal{O}_{T^{\vec{x}}}\right) \\
& =0 .
\end{aligned}
$$

Since $\mathcal{L}_{Y}$ has rank one, by 2.9 (see also [VdB, 3.5.4]), this implies that in the fundamental cycle of $\pi$, the middle curve has coefficient one. In (2.B), this implies that $\beta-v \geq 0$, and thus the fundamental cycle is reduced by the paragraph following (2.B).

In the sequel, we require the following description of some degenerate cases.

Lemma 3.16. Let $0 \neq \vec{x} \in \mathbb{L}_{+}$and write $\vec{x}=\sum_{i=1}^{n} a_{i} \vec{x}_{i}+a \vec{c}$ with $a \geq 0$ in normal form.

(1) If all $a_{i}=0$ (so necessarily $a>0$ ), then $Y^{\vec{x}}=T^{\vec{x}}=\mathcal{O}_{\mathbb{P}^{1}}(-a)$ and $S^{\vec{x}}=\mathbb{k}[x, y]^{\frac{1}{a}(1,1)}$.

(2) If $a_{i} \neq 0$ and $a_{j}=0$ for all $j \neq i$, then the dual graph of $\pi$ in (3.K) is

$$
\stackrel{\bullet-a}{-1-\alpha_{i 1}} \cdot \cdots \overline{-\alpha_{i m_{i}-1}} \cdot \overline{-\alpha} \cdot \dot{ }_{i m_{i}}
$$

$$
\text { where } \frac{p_{i}}{p_{i}-a_{i}}=\left[\alpha_{i 1}, \ldots, \alpha_{i m_{i}}\right]
$$

Proof. (1) By $2.4 S^{\vec{c}}=\mathbb{k}\left[t_{0}, t_{1}\right]$, and hence $S^{a \vec{c}}$ is the $a$-th Veronese of $\mathbb{k}\left[t_{0}, t_{1}\right]$, which is $\mathbb{k}[x, y]^{\frac{1}{a}(1,1)}$. Since $\left(S_{t_{i}}[t]\right)_{0}=\mathbb{k}\left[\frac{t_{1-i}}{t_{i}}, t_{i}^{a} t\right]$, the description (3.A) of $T^{\vec{x}}$ coincides with that of $\mathcal{O}_{\mathbb{P}^{1}}(-a)$. Thus $\mathcal{O}_{\mathbb{P}^{1}}(-a)=T^{\vec{x}}=Y^{\vec{x}}$. (2) There is only one singularity in (3.B), which implies that the dual graph has the above Type $A$ form. It is standard that $\alpha_{i j}$ from $\frac{p_{i}}{p_{i}-a_{i}}=\left[\alpha_{i 1}, \ldots, \alpha_{i m_{i}}\right]$ resolves $\frac{1}{p_{i}}\left(1,-a_{i}\right)$, and thus the only thing still to be verified is the self-intersection number $-1-a$. There are two ways of doing this: since the fundamental cycle of $\pi$ is reduced by 3.15 , the reconstruction algebra is easy to calculate and it can be directly verified that its quiver has the form given by intersection rules in 2.12 (which, by [W2], hold for non-minimal resolutions too). Alternatively, the number $-1-a$ can be determined by an explicit gluing calculation on $T^{\vec{x}}$; in both cases we suppress the details.

\subsection{Special CM Modules and the Dual Graph}

Choose $0 \neq \vec{x} \in \mathbb{L}_{+}$. In this subsection we first give a precise criterion for when $\pi: Y^{\vec{x}} \rightarrow \operatorname{Spec} S^{\vec{x}}$ in $(3 . K)$ is the minimal resolution, then we use the results of the previous subsections to determine the indecomposable special CM $S^{\vec{x}}$-modules.

\section{Proposition 3.17. Let $0 \neq \vec{x} \in \mathbb{L}_{+}$. Then $\pi$ is the minimal resolution if and only if $\vec{x} \notin[0, \vec{c}]$.}

Proof. Write $\vec{x}=\sum_{i=1}^{n} a_{i} \vec{x}_{i}+a \vec{c}$ in normal form, then since $\vec{x} \in \mathbb{L}_{+}$, necessarily $a \geq 0$. As in 3.15 , resolving the singularities in (3.B) it is clear that the dual graph of $\pi$ is star shaped, and the only curve that might be a $(-1)$-curve is the middle one.

$(\Leftarrow)$ Suppose that $\vec{x} \notin[0, \vec{c}]$. If all $a_{i}=0$ then necessarily $a \geq 2$, and so 3.16(1) shows that $\pi$ is the minimal resolution. Similarly, if $a_{i} \neq 0$ but $a_{j}=0$ for all $j \neq i$, then the assumption $\vec{x} \notin[0, \vec{c}]$ forces $a \geq 1$, and 3.16(2) then shows that $\pi$ is minimal.

Hence we can assume that $\vec{x} \notin[0, \vec{c}]$ with at least two of the $a_{i}$ being non-zero. This being the case, there are at least two singular points in (3.B). By 3.15, since the fundamental cycle is reduced, the calculation (2.B) shows that the middle curve then cannot be a $(-1)$-curve, hence the resolution is minimal.

$\Leftrightarrow)$ By contrapositive, suppose that $0 \neq \vec{x} \in[0, \vec{c}]$, say $\vec{x}=a_{i} \vec{x}_{i}$ for some $i$ and some $0<a_{i}<p_{i}$. Since $a=0$, by 3.16(2) the resolution $\pi$ is not minimal. 
Hence if $x \in \mathbb{L}_{+}$with $x \notin[0, \vec{c}]$, it follows that the dual graph of the minimal resolution $\pi: Y^{\vec{x}} \rightarrow$ Spec $S^{\vec{x}}$ is (1.E), except that we have not yet determined the precise value of $\beta$. We will do this later in 4.19 , since for the moment this value is not needed. As notation, for $\vec{y} \in \mathbb{L}$ write $S(\vec{y})^{\vec{x}}:=\bigoplus_{i \in \mathbb{Z}} S_{\vec{y}+i \vec{x}}$.

Theorem 3.18. Suppose that $\vec{x} \in \mathbb{L}_{+}$with $\vec{x} \notin[0, \vec{c}]$, and write $\vec{x}=\sum_{i=1}^{n} a_{i} \vec{x}_{i}+a \vec{c}$ with $a \geq 0$ in normal form. Then

$$
\operatorname{SCM} S^{\vec{x}}=\operatorname{add}\left\{S\left(u \vec{x}_{j}\right)^{\vec{x}} \mid j \in[1, n], u \in I\left(p_{j}, p_{j}-a_{j}\right)\right\} .
$$

Proof. The ring $S^{\vec{x}}=S^{\mathbb{N} \vec{x}}$ has a unique singular point corresponding to the graded maximal ideal by 2.3(3). Thus, by 2.6 we may complete $S^{\vec{x}}$ at this point and pass to the formal fibre, which is still the minimal resolution. However, to aid readability, we do not add $\widehat{(-)}$ to the notation.

Consider the bundle $q^{*} \mathcal{E}$ on $\mathbb{T}$, and its pushdown $g_{*} q^{*} \mathcal{E}$ on $T^{\vec{x}}$. At the point $\lambda_{1}$ of $T^{\vec{x}}$, which is the singularity $\frac{1}{p_{1}}\left(1,-a_{1}\right)$ by 3.2 , the sheaves

$$
g_{*} q^{*} \mathcal{O}, g_{*} q^{*} \mathcal{O}\left(\vec{x}_{1}\right), \ldots, g_{*} q^{*} \mathcal{O}\left(\left(p_{1}-1\right) \vec{x}_{1}\right)
$$

are all locally free away from the point $\lambda_{1}$, since at any other singular point $\lambda_{i}$, multiplication by $x_{1}$ is invertible. Further, at the point $\lambda_{1}$, (3.L) is a full list of the CM modules, indexed by the characters of $\mathbb{Z}_{p_{1}}=\frac{1}{p_{1}}\left(1,-a_{1}\right)$ in the obvious way. Hence by 2.17 , which does not require any coprime assumption, the torsion-free pullbacks under $\varphi$ of

$$
\left\{g_{*} q^{*} \mathcal{O}\left(u \vec{x}_{1}\right) \mid u \in I\left(p_{1}, p_{1}-a_{1}\right) \backslash\left\{0, p_{1}\right\}\right\}
$$

are precisely the line bundles on $Y^{\vec{x}}$ corresponding to the curves in arm 1 of the dual graph. By 2.9 and 3.15 they are the special bundles on $Y^{\vec{x}}$ corresponding to the curves in arm 1 of the dual graph, hence their pushdown (via $\pi$ ) to $S^{\vec{x}}$ are the special CM $S^{\vec{x}}$-modules corresponding to arm 1. Since the pushdown under $\varphi$ of the torsion-free pullback of $\varphi$ is the identity, the pushdown to $S^{\vec{x}}$ gives the modules

$$
\left\{\gamma_{*} g_{*} q^{*} \mathcal{O}\left(u \vec{x}_{1}\right) \mid u \in I\left(p_{1}, p_{1}-a_{1}\right) \backslash\left\{0, p_{1}\right\}\right\} .
$$

Then, by 3.9(1), $\gamma_{*} g_{*} q^{*} \mathcal{O}\left(u \vec{x}_{1}\right)=\bigoplus_{i>0} S_{i \vec{x}+u x_{1}}$. But since $\vec{x} \in \mathbb{L}_{+}, \vec{x} \notin[0, \vec{c}]$ and $u \leq p_{1}$ we see that $u x_{1}-\vec{x} \leq \vec{c}-\vec{x} \geq 0$, and hence $\gamma_{*} g_{*} q^{*} \mathcal{O}\left(u \vec{x}_{1}\right)=\bigoplus_{i \in \mathbb{Z}} S_{i \vec{x}+u x_{1}}:=S\left(u \vec{x}_{1}\right)^{\vec{x}}$.

The argument for the other arms is identical. The argument that the middle curve gives the special CM module $S(\vec{c})^{\vec{x}}$ follows again by 3.15 .

Remark 3.19. It is possible to assign each special CM $S^{\vec{x}}$-module to its vertex in the dual graph of the minimal resolution across the bijection in 2.9 , see below 3.20 for a typical example. As in 3.20 , there are obvious irreducible morphisms between the special CM $S^{\vec{x}}$-modules, so they must appear in the quiver of the reconstruction algebra. By the intersection theory in 2.12, we conclude that $S(\vec{c})^{\vec{x}}$ corresponds to the middle vertex, and this forces the positions of the other special CM modules relative to the dual graph.

Example 3.20. Consider the example $\left(p_{1}, p_{2}, p_{3}\right)=(3,5,5)$ and $\vec{x}=2 \vec{x}_{1}+2 \vec{x}_{2}+3 \vec{x}_{3}$. The continued fractions for $\frac{p_{i}}{p_{i}-a_{i}}$, and the corresponding $i$-series are given by:

$$
\begin{array}{ll}
\frac{3}{3-2}=[3] & 3>1>0 \\
\frac{5}{5-2}=[2,3] & 5>3>1>0 \\
\frac{5}{5-3}=[3,2] & 5>2>1>0
\end{array}
$$


It follows from 3.18 that an additive generator of $\mathrm{SCM} S^{\vec{x}}$ is given by the direct sum of the following circled modules:

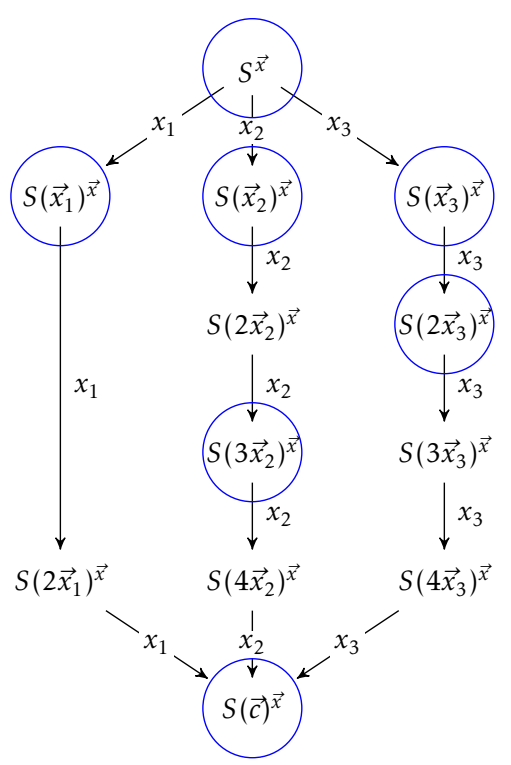

Consider the tilting bundle $\mathcal{M}$ on $Y^{\vec{x}}$, generated by global sections, constructed in [VdB, 3.5.4].

Corollary 3.21. If $0 \neq \vec{x} \in \mathbb{L}_{+}$, then the following statements hold.

(1) $\pi_{*} \mathcal{M}$ is a summand of $\bigoplus_{\vec{y} \in[0, \vec{c}]} S(\vec{y})^{\vec{x}}=\gamma_{*} g_{*}\left(q^{*} \mathcal{E}\right)$.

(2) There is an idempotent $e \in \operatorname{End}_{\mathbb{T}^{\vec{x}}}\left(q^{*} \mathcal{E}\right)$ such that $e \operatorname{End}_{\mathbb{T}^{\vec{x}}}\left(q^{*} \mathcal{E}\right) e \cong \operatorname{End}_{Y^{\vec{x}}}(\mathcal{M})$.

(3) There is a fully faithful embedding

$$
\mathrm{D}^{\mathrm{b}}\left(\operatorname{coh} Y^{\vec{x}}\right) \hookrightarrow \mathrm{D}^{\mathrm{b}}\left(\operatorname{coh} \mathbb{T}^{\vec{x}}\right)
$$

Proof. (1) By 3.15 the fundamental cycle is reduced. It follows that $\pi_{*} \mathcal{M}$ is a summand of $\bigoplus_{\vec{y} \in[0, \vec{c}]} S(\vec{y})^{\vec{x}}$, by the argument in the proof of 3.18 .

(2) Even although $\pi: Y^{\vec{x}} \rightarrow \operatorname{Spec} S^{\vec{x}}$ need not be the minimal resolution, it is still true by [DW, 4.3] that

$$
\operatorname{End}_{Y^{\vec{x}}}(\mathcal{M}) \cong \operatorname{End}_{S_{\vec{x}}}\left(\pi_{*} \mathcal{M}\right)
$$

On the other hand,

$$
\operatorname{End}_{\mathbb{T}^{\vec{x}}}\left(q^{*} \mathcal{E}\right) \cong \operatorname{End}_{S^{\vec{x}}}\left(\gamma_{*} g_{*} q^{*} \mathcal{E}\right) \cong \operatorname{End}_{S^{\vec{x}}}\left(\bigoplus_{\vec{y} \in[0, \vec{c}]} S(\vec{y})^{\vec{x}}\right) .
$$

Thus by (1) there is an idempotent $e \in \operatorname{End}_{\mathbb{T}^{\vec{x}}}\left(q^{*} \mathcal{E}\right)$ such that $e \operatorname{End}_{\mathbb{T}^{\vec{x}}}\left(q^{*} \mathcal{E}\right) e \cong \operatorname{End}_{Y^{\vec{x}}}(\mathcal{M})$.

(3) By (2), writing $A:=\operatorname{End}_{\mathbb{T}^{\vec{x}}}\left(q^{*} \mathcal{E}\right)$ then $\operatorname{End}_{Y \vec{x}}(\mathcal{M})=e A e$, thus there is an obvious embedding of derived categories

$$
\operatorname{RHom}_{e A e}(A e,-): \mathrm{D}\left(\operatorname{ModEnd}_{Y^{\vec{x}}}(\mathcal{M})\right) \hookrightarrow \mathrm{D}\left(\operatorname{ModEnd}_{\mathbb{T}^{\vec{x}}}\left(q^{*} \mathcal{E}\right)\right),
$$

and also an embedding given by $-\otimes_{e A e}^{\mathrm{L}} e A$. Regardless, since gl.dim $e A e<\infty$, the above induces an embedding

$$
\mathrm{D}^{\mathrm{b}}\left(\operatorname{modEnd}_{Y^{\vec{x}}}(\mathcal{M})\right) \hookrightarrow \mathrm{D}^{\mathrm{b}}\left(\operatorname{modEnd}_{\mathbb{T}^{\vec{x}}}\left(q^{*} \mathcal{E}\right)\right) .
$$

The left hand side is equivalent to $\mathrm{D}^{\mathrm{b}}\left(\operatorname{coh} Y^{\vec{x}}\right)$, so it suffices to show that the right hand side is equivalent to $\mathrm{D}^{\mathrm{b}}\left(\operatorname{coh} \mathbb{T}^{\vec{x}}\right)$. By 3.13 , there is an unbounded derived equivalence $\mathrm{D}\left(\operatorname{ModEnd}_{\mathbb{T}^{\vec{x}}}\left(q^{*} \mathcal{E}\right)\right) \simeq \mathrm{D}\left(\mathrm{Q} \operatorname{coh} \mathbb{T}^{\vec{x}}\right)$. This automatically restricts to an equivalence on compact objects. The compact objects of $\mathrm{D}\left(\mathrm{Q}\right.$ coh $\left.\mathbb{T}^{\vec{x}}\right)$ are $\mathrm{D}^{\mathrm{b}}\left(\operatorname{coh} \mathbb{T}^{\vec{x}}\right)$ by [BLS, A.3], and since $\operatorname{End}_{\mathbb{T}^{\vec{x}}}\left(q^{*} \mathcal{E}\right)$ has finite global dimension, the compact objects of $\mathrm{D}\left(\operatorname{ModEnd}_{\mathbb{T}^{\vec{x}}}\left(q^{*} \mathcal{E}\right)\right)$ are $\mathrm{D}^{\mathrm{b}}\left(\operatorname{modEnd}_{\mathbb{T}^{\vec{x}}}\left(q^{*} \mathcal{E}\right)\right)$, as required. 
We give a simple criterion for when the above is an equivalence later in 4.8. Note that the above result is formally very similar to the case of quotient singularities, where the reconstruction algebra embeds into the quotient stack $\left[\mathrm{k}^{2} / G\right]$, but this embedding is also very rarely an equivalence.

\section{Categorical Equivalences}

In this section we investigate the conditions on $\vec{x}$ under which

$$
\operatorname{coh} \mathbb{X} \simeq \operatorname{qgr}^{\mathbb{Z}} S^{\vec{x}} \simeq \operatorname{qgr}^{\mathbb{Z}} \Gamma_{\vec{x}}
$$

holds. This allows us, in 4.8, to give a precise criterion for when the embedding in 3.21(3) is an equivalence, and further it allows us in 4.19 to determine the middle self-intersection number in (1.E). Throughout many results in this section, a coprime condition $\left(p_{i}, a_{i}\right)=1$ naturally appears, and in $\S 4.3$ we show that we can always change parameters so that this coprime condition holds.

\subsection{General Results on Categorical Equivalences}

To simplify the notation, in this subsection we first produce categorical equivalences in a very general setting, before specialising in the next subsection to the case of the weighted projective line. Throughout this subsection, $k$ denotes an arbitrary field.

We start with a basic observation. Let $G$ be an abelian group and $A$ a noetherian $G$-graded $k$-algebra. As in $\$ 1.3$ we consider the categories $\bmod ^{G} A, \bmod _{0}^{G} A$ and $\mathrm{qgr}^{G} A$. For an idempotent $e \in A_{0}, B:=e A e$ is a noetherian $G$-graded $k$-algebra. The functor

$$
E:=e(-): \bmod ^{G} A \rightarrow \bmod ^{G} B
$$

has a left adjoint functor $E_{\lambda}$ and a right adjoint functor $E_{\rho}$ given by

$$
\begin{array}{r}
E_{\lambda}:=A e \otimes_{B}-: \bmod ^{G} B \rightarrow \bmod ^{G} A \\
E_{\rho}:=\operatorname{Hom}_{B}(e A,-): \bmod ^{G} B \rightarrow \bmod ^{G} A .
\end{array}
$$

Moreover $E E_{\lambda}=\mathrm{id}_{\bmod ^{G} B}=E E_{\rho}$ holds, and for the natural morphism $m: A e \otimes_{B} e A \rightarrow A$, the counit $\eta: E_{\lambda} E \rightarrow \operatorname{id}_{\bmod ^{G} A}$ is given by $m \otimes_{A}-$ and the unit $\varepsilon: \operatorname{id}_{\bmod ^{G} A} \rightarrow E_{\rho} E$ is given by $\operatorname{Hom}_{A}(m,-)$.

The following basic observation is a prototype of our results in this subsection.

Proposition 4.1. If $\operatorname{dim}_{k} A /(e)<\infty$, then $E$ induces an equivalence $\mathrm{qgr}^{G} A \simeq \mathrm{qgr}^{G} B$.

Proof. Clearly $E_{\lambda}$ and $E$ induce an adjoint pair $E_{\lambda}: \mathrm{qgr}^{G} A \rightarrow \mathrm{qgr}^{G} B$ and $E: \mathrm{qgr}^{G} B \rightarrow \operatorname{qgr}^{G} A$. For any $X \in \bmod ^{G} A$, both the kernel and cokernel of $m \otimes_{A} X: E_{\lambda} E X \rightarrow X$ are finite dimensional since they are finitely generated $(A /(e))$-modules. Therefore $E_{\lambda}$ and $E$ give the desired equivalences.

In the rest of this subsection, let $G$ be an abelian group and $H$ a subgroup of $G$ of finite index. Assume that $A$ is a noetherian $G$-graded $k$-algebra, and let $B:=A^{H}=\bigoplus_{g \in H} A_{g}$ be the $H$-Veronese subring of $A$. There is a natural functor

$$
(-)^{H}: \bmod ^{G} A \rightarrow \bmod ^{H} B
$$

given by $X^{H}:=\bigoplus_{h \in H} X_{h}$.

Lemma 4.2. $B$ is a noetherian $k$-algebra and $A$ is a finitely generated B-module.

Proof. There is a finite direct sum decomposition $A=\bigoplus_{g \in G / H} A(g)^{H}$ as $B$-modules. For any submodule $M$ of $A(g)^{H}$, it is easy to check that the ideal $A M$ of $A$ satisfies $A M \cap A(g)^{H}=M$. Therefore $A(g)^{H}$ is a noetherian $B$-module, since $A$ is a noetherian ring. The assertion follows. 
We say that $X \in \bmod ^{G} A$ has depth at least two if $\operatorname{Ext}_{A}^{i}(Y, X)=0$ for any $i=0,1$ and $Y \in \bmod ^{G} A$ with $\operatorname{dim}_{k} Y<\infty$. We write $\bmod _{2}^{G} A$ for the full subcategory of $\bmod ^{G} A$ consisting of modules with depth at least two. We define $\bmod _{2}^{H} B$ similarly.

Theorem 4.3. Let $G$ be an abelian group, $H$ a subgroup of $G$ of finite index, $A$ a noetherian $G$-graded $k$-algebra, and $B:=A^{H}$. Then the following conditions are equivalent.

(1) The natural functor $(-)^{H}: \mathrm{qgr}^{G} A \rightarrow \mathrm{qgr}^{H} B$ is an equivalence.

(2) For any $i \in G$, the ideal $I^{i}:=A(i)^{H} \cdot A(-i)^{H}$ of $B$ satisfies $\operatorname{dim}_{k}\left(B / I^{i}\right)<\infty$.

If $A$ belongs to $\bmod _{2}^{G} A$, then the following condition is also equivalent.

(3) The natural functor $(-)^{H}: \bmod _{2}^{G} A \rightarrow \bmod _{2}^{H} B$ is an equivalence.

Proof. Consider the matrix algebra

$$
C=\left(A(i-j)^{H}\right)_{i, j \in G / H}
$$

whose rows and columns are indexed by $G / H$, and the product is given by the matrix multiplication together with the product in $A$, namely

$$
\left(s_{i, j}\right) \cdot\left(t_{i, j}\right):=\left(\sum_{k \in G / H} s_{i, k} \cdot t_{k, j}\right) .
$$

Now we fix a complete set $I$ of representatives of $G / H$ in $G$. Then $C$ has an $H$-grading given by

$$
C_{h}:=\left(A_{i-j+h}\right)_{i, j \in I} .
$$

By [IL, Theorem 3.1] there is an equivalence

$$
F: \bmod ^{G} A \simeq \bmod ^{H} C
$$

sending $M=\bigoplus_{i \in G} M_{i}$ to $F(M)=\bigoplus_{h \in H} F(M)_{h}$, where $F(M)_{h}$ is defined by

$$
F(M)_{h}:=\left(M_{i+h}\right)_{i \in I}
$$

and the $C$-module structure is given by

$$
\left(s_{i, j}\right)_{i, j \in I} \cdot\left(m_{i}\right)_{i \in I}:=\left(\sum_{j \in I} s_{i, j} \cdot m_{j}\right)_{i \in I} .
$$

On the other hand, let $e \in C$ be the idempotent corresponding to $0 \in G / H$. Since $e C e=B$ holds, there is an exact functor

$$
E:=e(-): \bmod ^{H} C \rightarrow \bmod ^{H} B
$$

such that the following diagram commutes

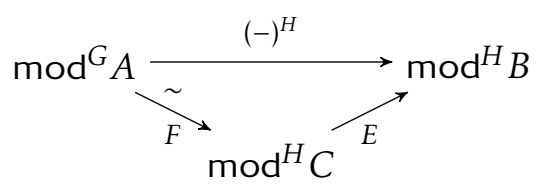

The functor (4.B) has a left adjoint functor $E_{\lambda}:=C e \otimes_{B}-: \bmod ^{H} B \rightarrow \bmod ^{H} C$ and a right adjoint functor $E_{\rho}:=\operatorname{Hom}_{B}(e C,-): \bmod ^{H} B \rightarrow \bmod ^{H} C$.

(1) $\Leftrightarrow(2)$ The functors $F$ and $E$ induce an equivalence $F: \operatorname{qgr}^{G} A \simeq \operatorname{qgr}^{H} C$ and a functor

$$
E: \mathrm{qgr}^{H} \mathrm{C} \rightarrow \mathrm{qgr}^{H} B
$$


respectively, which make the following diagram commutative

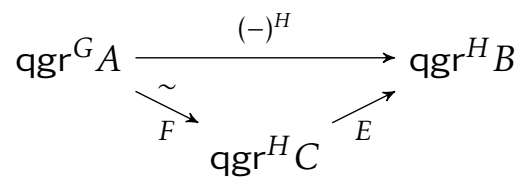

Thus the functor $(-)^{H}: \mathrm{qgr}^{G} A \rightarrow \mathrm{qgr}^{H} B$ is an equivalence if and only if the functor (4.C) is an equivalence. The functor $E_{\lambda}: \bmod ^{H} B \rightarrow \bmod ^{H} C$ induces a left adjoint functor $E_{\lambda}: \mathrm{qgr}^{H} B \rightarrow \mathrm{qgr}^{H} C$ of (4.C). Clearly $E E_{\lambda}=\mathrm{id}_{\mathrm{qgr}^{H} B}$ holds, and the counit $\eta: E_{\lambda} E \rightarrow \mathrm{id}_{\mathrm{qgr}^{H} C}$ is given by $m \otimes_{C}-$, where $m$ is the natural morphism

$$
m: C e \otimes_{B} e C \rightarrow C .
$$

Thus the condition (1) holds if and only if $\eta$ is an isomorphism of functors if and only if $m$ is an isomorphism in $\mathrm{qgr}^{\mathrm{H}} \mathrm{C}$. On the other hand, the cokernel of $m$ is $C /(e)$, where $(e)$ is the two-sided ideal of $C$ generated by $e$, and the kernel of $m$ is a finitely generated $C /(e)$-module. Therefore (1) holds if and only if the factor algebra $C /(e)$ of $C$ is finite dimensional if and only if (2) holds, by the following observation.

Lemma 4.4. $\operatorname{dim}_{k} C /(e)<\infty$ if and only if the condition (2) holds.

Proof of Lemma 4.4. Since

$$
C /(e)=\left(A(i-j)^{H} /\left(A(i)^{H} \cdot A(-j)^{H}\right)\right)_{i, j \in I}
$$

holds, $C /(e)$ is finite dimensional if and only if $A(i-j)^{H} /\left(A(i)^{H} \cdot A(-j)^{H}\right)$ is finite dimensional for any $i, j \in I$. This implies the condition (2) by considering the case $i=j$.

Conversely assume that (2) holds. Since there is a surjective map

$$
A(i-j)^{H} \otimes_{B} \frac{B}{A(j)^{H} \cdot A(-j)^{H}}=\frac{A(i-j)^{H}}{A(i-j)^{H} \cdot A(j)^{H} \cdot A(-j)^{H}} \rightarrow \frac{A(i-j)^{H}}{A(i)^{H} \cdot A(-j)^{H}}
$$

whose domain is finite dimensional, the target is also finite dimensional. Thus the assertion holds.

$(2) \Leftrightarrow(3)$ Assume that $A \in \bmod _{2}^{G} A$. Clearly the equivalence (4.A) induces equivalences

$$
F: \bmod _{0}^{G} A \simeq \bmod _{0}^{H} C \text { and } F: \bmod _{2}^{G} A \simeq \bmod _{2}^{H} C \text {. }
$$

The remainder of the proof requires the following general lemma.

\section{Lemma 4.5. With the setup as above,}

(1) The functor (4.B) induces a functor

$$
E: \bmod _{2}^{H} C \rightarrow \bmod _{2}^{H} B .
$$

(2) The functor $E_{\rho}: \bmod ^{H} B \rightarrow \bmod ^{H} C$ induces a functor $E_{\rho}: \bmod _{2}^{H} B \rightarrow \bmod _{2}^{H} C$.

(3) $X \in \bmod ^{H} C$ belongs to $\bmod _{0}^{H} C$ if and only if $\operatorname{Ext}_{C}^{i}\left(X, \bmod _{2}^{H} C\right)=0$ for $i=0,1$.

Proof of Lemma 4.5. (1) Let $X \in \bmod _{2}^{H} C, Y \in \bmod _{0}^{H} B$ and $\mathbf{E}_{\lambda} Y:=C e \stackrel{\mathbf{L}}{\otimes_{B}} Y$. Since $H^{i}\left(\mathbf{E}_{\lambda} Y\right)$ is zero for any $i>0$ and belongs to $\bmod _{0}^{H} C$ for any $i \leq 0$, we have $\operatorname{Hom}_{\mathrm{D}^{\mathrm{b}}(\bmod C)}\left(\mathbf{E}_{\lambda} Y, X[i]\right)=0$ for $i=0,1$. Using $\mathbf{R H o m}_{B}(Y, E X)=\mathbf{R H o m}_{C}\left(\mathbf{E}_{\lambda} Y, X\right)$, we have $\operatorname{Ext}_{B}^{i}(Y, E X)=0$ for $i=0,1$.

(2) Let $X \in \bmod _{2}^{H} B, Y \in \bmod _{0}^{H} C$ and $\mathbf{E}_{\rho} X:=\mathbf{R H o m}_{B}(e C, X)$. Since $\mathbf{R H o m}_{C}\left(Y, \mathbf{E}_{\rho} X\right)=\mathbf{R H o m}_{B}(E Y, X)$ and $E Y \in \bmod _{0}^{H} B$ hold, we have $\operatorname{Hom}_{D^{\mathrm{b}}(\bmod C)}\left(Y, \mathbf{E}_{\rho} X[i]\right)=0$ for $i=0,1$. There is a triangle

$$
E_{\rho} X \rightarrow \mathbf{E}_{\rho} X \rightarrow Z \rightarrow E_{\rho} X[1]
$$


satisfying $H^{i}(Z)=0$ for all $i \leq 0$. Applying $\operatorname{Hom}_{\mathrm{D}^{\mathrm{b}}(\bmod C)}(Y,-)$ gives $\operatorname{Ext}_{C}^{i}\left(Y, E_{\rho} X\right)=0$ for $i=0,1$.

(3) It suffices to prove the 'if' part. Our assumption $A \in \bmod _{2}^{G} A$ implies $C=\bigoplus_{i \in I} F(A(-i)) \in \bmod _{2}^{H} C$, since $C e_{j}=\left(A(i-j)^{H}\right)_{i \in I}=F(A(-j))$. Let $0 \rightarrow T \rightarrow X \rightarrow F \rightarrow 0$ and $0 \rightarrow \Omega F \rightarrow P \rightarrow F \rightarrow 0$ be exact sequences in $\bmod ^{H} C$ such that $T$ is the largest submodule of $X$ which belongs to $\bmod _{0}^{H} C$ and $P$ is an $H$-graded projective $C$-module. Then $\Omega F$ belongs to $\bmod _{2}^{H} C$ since $C \in \bmod _{2}^{H} C$. Applying $\operatorname{Hom}_{C}(-, \Omega F)$ to the first sequence gives an exact sequence

$$
0=\operatorname{Hom}_{C}(T, \Omega F) \rightarrow \operatorname{Ext}_{C}^{1}(F, \Omega F) \rightarrow \operatorname{Ext}_{C}^{1}(X, \Omega F)=0 .
$$

Thus $\operatorname{Ext}_{C}^{1}(F, \Omega F)=0$ holds, and $F$ is projective in $\bmod ^{H} C$. Hence $X=T \oplus F$, and so $\operatorname{Hom}_{C}(X, C)=0$ implies that $F=0$. Therefore $X=T$ belongs to $\bmod _{0}^{H} C$.

It follows from 4.5 that there is a commutative diagram

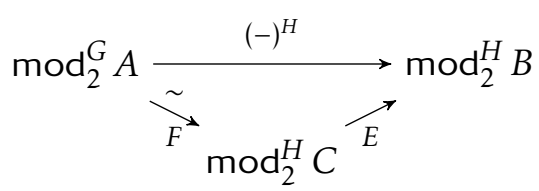

Thus the functor $(-)^{H}: \bmod _{2}^{G} A \rightarrow \bmod _{2}^{H} B$ is an equivalence if and only if the functor (4.E) is an equivalence. By 4.5(2), there is a right adjoint functor $E_{\rho}: \bmod _{2}^{H} B \rightarrow \bmod _{2}^{H} C$ of (4.E). Clearly $E E_{\rho}=\operatorname{id}_{\bmod _{2}^{H} B}$ holds, and the unit $\varepsilon: \operatorname{id}_{\bmod _{2}^{H} C} \rightarrow E_{\rho} E$ is given by $\operatorname{Hom}_{C}(m,-)$, where $m$ is the morphism (4.D). Thus the condition (3) holds if and only if $\varepsilon=\operatorname{Hom}_{C}(m,-)$ is an isomorphism of functors.

Now fix $X \in \bmod _{2}^{H} C$ and apply $\operatorname{Hom}_{C}(-, X)$ to exact sequences $0 \rightarrow(e) \rightarrow C \rightarrow C /(e) \rightarrow 0$ and $0 \rightarrow \mathrm{Ker} m \rightarrow \mathrm{Ce} \otimes_{B} e C \rightarrow(e) \rightarrow 0$. This gives exact sequences

$$
\begin{gathered}
0 \rightarrow \operatorname{Hom}_{C}(C /(e), X) \rightarrow X \rightarrow \operatorname{Hom}_{C}((e), X) \rightarrow \operatorname{Ext}_{C}^{1}(C /(e), X) \rightarrow 0 \\
0 \rightarrow \operatorname{Hom}_{C}((e), X) \rightarrow \operatorname{Hom}_{C}\left(C e \otimes_{B} e C, X\right) \rightarrow \operatorname{Hom}_{C}(\operatorname{Ker} m, X) .
\end{gathered}
$$

Therefore, if $C /(e)$ is finite dimensional, then so is $\operatorname{Ker} m$ and hence $\varepsilon$ is an isomorphism. Conversely, if $\varepsilon$ is an isomorphism, then $\operatorname{Ext}_{C}^{i}(C /(e), X)=0$ for $i=0,1$ for any $X \in \bmod _{2}^{H} C$ and hence $C /(e)$ is finite dimensional by 4.5(3). Consequently (3) is equivalent to (2), again by 4.4.

Later we need the following observation.

Lemma 4.6. In the setting of 4.3, assume that the condition (2) is satisfied. Then for any $X \in \bmod ^{G} A$ and $Y \in \bmod _{2}^{G} A$, there is an isomorphism

$$
\operatorname{Hom}_{B}\left(X^{H}, Y^{H}\right) \cong \operatorname{Hom}_{A}(X, Y)^{H}
$$

of H-graded $k$-modules.

Proof. Clearly $\operatorname{Hom}_{B}\left(X^{H}, Y^{H}\right)=\operatorname{Hom}_{B}(E F X, E F Y)=\operatorname{Hom}_{C}\left(E_{\lambda} E F X, F Y\right)$. This is isomorphic to $\operatorname{Hom}_{C}(F X, F Y)$ since the kernel and the cokernel of $\eta_{X}: E_{\lambda} E X \rightarrow X$ are finite dimensional by our assumptions. Finally,

$$
\operatorname{Hom}_{C}(F X, F Y)=\bigoplus_{h \in H} \operatorname{Hom}_{C}^{H}(F X,(F Y)(h))=\bigoplus_{h \in H} \operatorname{Hom}_{A}^{G}(X, Y(h))=\operatorname{Hom}_{A}(X, Y)^{H} .
$$




\subsection{Categorical Equivalences for Weighted Projective Lines}

In this subsection, we apply the general results of the previous subsection to describe the precise conditions on $\vec{x} \in \mathbb{L}$ for which qgr $\mathbb{Z}^{\vec{x}} \simeq$ coh $\mathbb{X}$ holds. As before, write $S(\vec{y})^{\vec{x}}:=\bigoplus_{i \in \mathbb{Z}} S_{\vec{y}+i \vec{x}}$. This subsection does not require the condition that $\vec{x}$ belongs to $\mathbb{L}_{+}$, instead assuming that $\vec{x}$ is not torsion. The following is the main result, where the special case $\vec{x}=\vec{\omega}$ was given in [GL2]. Another approach can be found in [H2].

Theorem 4.7. If $\vec{x}=\sum_{i=1}^{n} a_{i} \vec{x}_{i}+a \vec{c} \in \mathbb{L}$ is not torsion, then the following conditions are equivalent.

(1) The natural functor $(-)^{\vec{x}}: \mathrm{CM}^{\mathbb{L}} S \rightarrow \mathrm{CM}^{\mathbb{Z}} S^{\vec{x}}$ is an equivalence.

(2) The natural functor $(-)^{\vec{x}}: \mathrm{qgr}^{\mathbb{L}} S \rightarrow \mathrm{qgr}^{\mathbb{Z}} S^{\vec{x}}$ is an equivalence.

(3) For any $\vec{z} \in \mathbb{L}$, the ideal $I^{\vec{z}}:=S(\vec{z})^{\vec{x}} \cdot S(-\vec{z})^{\vec{x}}$ of $S^{\vec{x}}$ satisfies $\operatorname{dim}_{\mathbb{k}}\left(S^{\vec{x}} / I^{\vec{z}}\right)<\infty$.

(4) $\left(p_{i}, a_{i}\right)=1$ for all $1 \leq i \leq n$.

Proof. To ease notation, write $R:=S^{\vec{x}}$.

$(1) \Leftrightarrow(2) \Leftrightarrow(3)$ These are shown in 4.3 since $C M^{\mathbb{L}} S=\bmod _{2}^{\mathbb{L}} S$ and $C M^{\mathbb{Z}} R=\bmod _{2}^{\mathbb{Z}} R$.

(3) $\Rightarrow(4)$. By contrapositive, assume that $a_{1}$ and $p_{1}$ are not coprime. Then the normal form of any element in $\vec{x}_{1}+\mathbb{Z} \vec{x}$ (respectively, $\left.-\vec{x}_{1}+\mathbb{Z} \vec{x}\right)$ contains a positive multiple of $\vec{x}_{1}$. Thus we have

$$
I^{\vec{x}_{1}} \subset S x_{1} \cdot S x_{1}=S x_{1}^{2} \text {. }
$$

Therefore the condition (3) implies that the algebra $R /\left(R \cap S x_{1}^{2}\right)$ is finite dimensional. Since $S / S x_{1}^{2}$ is a finitely generated $R /\left(R \cap S x_{1}^{2}\right)$-module by 2.3(1), it is also finite dimensional. This is a contradiction since $S$ has Krull dimension two.

(4) $\Rightarrow(3)$. Assume that $\left(p_{i}, a_{i}\right)=1$ for all $i$. If $R / I^{\vec{y}}$ and $R / I^{\vec{z}}$ are finite dimensional, then so is $R / I^{\vec{y}+\vec{z}}$ since $I^{\vec{y}} \cdot I^{\vec{z}} \subset I^{\vec{y}+\vec{z}}$ holds. Thus we only have to show that $R / I^{\vec{x}_{i}}$ is finite dimensional for each $i$ with $1 \leq i \leq n$. We will show that $I^{\vec{x}_{i}}$ contains a certain power $A$ of $x_{i}$ and a certain monomial $B$ of $x_{j}$ 's with $j \neq i$. Then it is easy to check that $S /(S A+S B)$ is finite dimensional, and hence $R /(R A+R B)=(S /(S A+S B))^{\vec{x}}$ and $R / I^{\vec{x}_{i}}$ are also finite dimensional.

For the least common multiple $p$ of $p_{1}, \ldots, p_{n}$, we have $p \vec{x}=q \vec{c}$ for some $q>0$. Then

$$
I^{\vec{x}_{i}}=S\left(\vec{x}_{i}\right)^{\vec{x}} \cdot S\left(-\vec{x}_{i}\right)^{\vec{x}} \supset S_{\vec{x}_{i}} \cdot S_{-\vec{x}_{i}+p \vec{x}} \ni x_{i} \cdot x_{i}^{p_{i} q-1}=x_{i}^{p_{i} q} .
$$

Thus $I^{\vec{x}_{i}}$ contains a power of $x_{i}$. On the other hand, since $a_{i}$ and $p_{i}$ are coprime, there exist integers $\ell$ and $m$ such that $a_{i} \ell+1=p_{i} m$ and $\vec{x}_{i}+\ell \vec{x} \in \mathbb{L}_{+}$. Then the normal form of $\vec{x}_{i}+\ell \vec{x}$ does not contain a positive multiple of $\vec{x}_{i}$, and hence $S\left(\vec{x}_{i}\right)^{\vec{x}} \supset S_{\vec{x}_{i}+\ell \vec{x}}$ contains a monomial of $x_{j}$ 's with $j \neq i$. Applying a similar argument to $S\left(-\vec{x}_{i}\right)^{\vec{x}}$, we have that $I^{\vec{x}_{i}}=S\left(\vec{x}_{i}\right)^{\vec{x}} \cdot S\left(-\vec{x}_{i}\right)^{\vec{x}}$ contains a monomial of $x_{j}$ 's with $j \neq i$. Thus the assertion follows.

The following is a geometric corollary of the results in this subsection.

Corollary 4.8. Suppose that $0 \neq \vec{x} \in \mathbb{L}_{+}$and write $\vec{x}=\sum_{i=1}^{n} a_{i} \vec{x}_{i}+a \vec{c}$ in normal form. If $n \geq 1$ and $\left(p_{i}, a_{i}\right)=1$ for all $1 \leq i \leq n$, then the fully faithful embedding

$$
\mathrm{D}^{\mathrm{b}}\left(\operatorname{coh} Y^{\vec{x}}\right) \hookrightarrow \mathrm{D}^{\mathrm{b}}\left(\operatorname{coh} \mathbb{T}^{\vec{x}}\right)
$$

in 3.21 is an equivalence if and only if every $a_{i}=1$, that is $\vec{x}=\sum_{i=1}^{n} x_{i}+a \vec{c}$.

Proof. We use the notation from the proof of 3.21. Note that from the assumption $\left(p_{i}, a_{i}\right)=1$ for every $1 \leq i \leq n$, necessarily each $a_{i}$ is non-zero. Next, the indecomposable summands of $\pi_{*} \mathcal{M}$ are pairwise nonisomorphic by combining [VdB, 3.5.3] and [DW, 4.3], and the summands of $\bigoplus_{\vec{y} \in[0, \vec{c}]} S(\vec{y})^{\vec{x}}$ are pairwise non-isomorphic by $4.7(1)$.

The embedding in 3.21 is induced from idempotents using the observation that $\pi_{*} \mathcal{M}$ is a summand of $\bigoplus_{\vec{y} \in[0, \vec{c}]} S(\vec{y})^{\vec{x}}$. It follows that the embedding is an equivalence if and only if for all $t=1, \ldots, n$, the $i$-series on arm $t$ has maximum length. By 2.16 this holds if and only if every $a_{i}=1$. 


\subsection{Changing Parameters}

Our next main result, 4.10 , shows that we can always change parameters, without changing the category of coherent sheaves, so that the condition $\left(p_{i}, a_{i}\right)=1$ for all $1 \leq i \leq n$ appearing in both $4.7(4)$ and 4.8 holds.

We now fix notation. Let $S:=S_{\mathbf{p}, \lambda}$, and fix a subset $I$ of $\{1, \ldots, n\}$. For each $i \in I$, choose a divisor $d_{i}$ of $p_{i}$. Let $\mathrm{p}_{i}:=p_{i} / d_{i}, \mathbf{p}^{\prime}:=\left(\mathrm{p}_{i} \mid i \in I\right), \lambda^{\prime}:=\left(\lambda_{i} \mid i \in I\right)$,

$$
S^{\prime}:=S_{\mathbf{p}^{\prime}, \lambda^{\prime}}=\frac{\mathbb{k}\left[\mathrm{t}_{0}, \mathrm{t}_{1}, \mathrm{x}_{i} \mid i \in I\right]}{\left(\mathrm{x}_{i}^{\mathrm{p}_{i}}-\ell_{i}\left(\mathrm{t}_{0}, \mathrm{t}_{1}\right) \mid i \in I\right)}
$$

and $\mathbb{L}^{\prime}:=\mathbb{L}\left(\mathrm{p}_{i} \mid i \in I\right)=\left\langle\vec{x}_{i}, \overrightarrow{\mathrm{c}} \mid i \in I\right\rangle /\left(\mathrm{p}_{i} \vec{x}_{i}-\vec{c} \mid i \in I\right)$. Then $S^{\prime}$ is an $\mathbb{L}^{\prime}$-graded $\mathbb{k}$-algebra, and there is an equivalence $\operatorname{coh} \mathbb{X}_{\mathbf{p}^{\prime}, \lambda^{\prime}}=$ qgr $^{\mathbb{L}^{\prime}} S^{\prime}$ as before.

Proposition 4.9. With notation as above,

(1) There is a monomorphism $\iota: \mathbb{L}^{\prime} \rightarrow \mathbb{L}$ of groups sending $\vec{x}_{i}$ to $d_{i} \vec{x}_{i}$ for each $i \in I$ and $\vec{c}$ to $\vec{c}$.

(2) There is a monomorphism $S^{\prime} \rightarrow S$ of $\mathbb{k}$-algebras sending $x_{i}$ to $x_{i}^{d_{i}}$ for each $i \in I$ and $\mathrm{t}_{j}$ to $t_{j}$ for $j=0,1$, which induces an isomorphism $S^{\prime} \simeq \bigoplus_{\overrightarrow{\mathrm{x}} \in \mathbb{L}^{\prime}} S_{l(\overrightarrow{\mathrm{x}})}$.

(3) Let $\vec{x} \in \mathbb{L}$ be an element with normal form $\vec{x}=\sum_{i \in I} a_{i} \vec{x}_{i}+a \vec{c}$ such that $a_{i}$ is a multiple of $d_{i}$. For $\mathrm{a}_{i}:=a_{i} / d_{i}$ and $\overrightarrow{\mathrm{x}}:=\sum_{i \in I} \mathrm{a}_{i} \vec{x}_{i}+a \overrightarrow{\mathrm{c}} \in \mathbb{L}^{\prime}$, we have $\left(S^{\prime}\right)^{\vec{x}}=S^{\vec{x}}$.

Proof. (1) Clearly $\iota$ is well-defined. Assume that $\vec{x} \in \mathbb{L}^{\prime}$ with normal form $\vec{x}=\sum_{i \in I} a_{i} \vec{x}_{i}+a \vec{c}$ belongs to the kernel of $\iota$. Then $0=\iota(\vec{x})=\sum_{i \in I} a_{i} d_{i} \vec{x}_{i}+a \vec{c}$, where the right hand side is a normal form in $\mathbb{L}$, and so $a_{i}=0=a$ for all $i$. Hence $\vec{x}=0$.

(2) Take any element $\vec{x} \in \mathbb{L}^{\prime}$ with a normal form $\vec{x}=\sum_{i \in I} a_{i} \vec{x}_{i}+a \vec{c}$. We prove $S_{\vec{x}}^{\prime} \simeq S_{l(\vec{x})}$. If $\vec{x} \notin \mathbb{L}_{+}^{\prime}$, then $\iota(\vec{x}) \notin \mathbb{L}_{+}$and both sides are zero. Assume $\vec{x} \in \mathbb{L}_{+}^{\prime}$. Then by $2.4, S_{\vec{x}}^{\prime}$ has a $\mathbb{k}$-basis

$$
\mathrm{t}_{0}^{j} \mathrm{t}_{1}^{a-j} \prod_{i \in I} \mathrm{x}_{i}^{a_{i}} \quad 0 \leq j \leq a .
$$

Since $\iota(\vec{x})$ has a normal form $\sum_{i \in I} a_{i} d_{i} \vec{x}_{i}+a \vec{c}$, it follows from 2.4 that $S_{\iota(\overrightarrow{\mathrm{x}})}$ has a $\mathbb{k}$-basis $t_{0}^{j} t_{1}^{a-j} \prod_{i \in I} x_{i}^{a_{i} d_{i}}$ for $0 \leq j \leq a$. The assertion follows.

(3) Immediate from (2).

Proposition 4.10. Suppose that $\vec{x} \in \mathbb{L}$ is not torsion, and write $\vec{x}=\sum_{i=1}^{n} a_{i} \vec{x}_{i}+a \vec{c} \in \mathbb{L}$ in normal form. Let $I:=\left\{1 \leq i \leq n \mid a_{i} \neq 0\right\}$, and consider the parameters $\left(\mathbf{p}^{\prime}, \lambda^{\prime}\right)$ defined by $\mathbf{p}^{\prime}:=\left(\mathrm{p}_{i} \mid i \in I\right)$ for $\mathrm{p}_{i}:=p_{i} /\left(a_{i}, p_{i}\right)$ and $\lambda^{\prime}:=\left(\lambda_{i} \mid i \in I\right)$. As above, set $\vec{x}:=\sum_{i \in I} a_{i} \vec{x}_{i}+a \vec{c} \in \mathbb{L}^{\prime}$, then the following statements hold.

(1) There is an isomorphism $S_{\mathbf{p}, \lambda}^{\vec{x}} \cong S_{\mathbf{p}^{\prime}, \lambda^{\prime}}^{\vec{x}}$ as $\mathbb{Z}$-graded $\mathbb{k}$-algebras.

(2) There are equivalences $\mathrm{CM}^{\mathbb{Z}} S_{\mathbf{p}, \lambda}^{\vec{x}} \simeq \mathrm{CM}^{\mathbb{Z}} S_{\mathbf{p}^{\prime}, \lambda^{\prime}}^{\vec{x}} \simeq \mathrm{CM}^{\mathbb{L}} S_{\mathbf{p}^{\prime}, \lambda^{\prime}}$.

(3) There are equivalences $\operatorname{qgr}^{\mathbb{Z}} S_{\mathbf{p}, \lambda}^{\vec{x}} \simeq \operatorname{qgr}^{\mathbb{Z}} S_{\mathbf{p}^{\prime}, \lambda^{\prime}}^{\vec{x}} \simeq \operatorname{coh} \mathbb{X}_{\mathbf{p}^{\prime}, \lambda^{\prime}}$.

Proof. Part (1) follows directly from 4.9(3). Certainly this induces the left equivalences in (2) and (3). Applying 4.7 to $S_{\mathbf{p}^{\prime}, \lambda^{\prime}}^{\vec{x}}$ gives the right equivalences in (2) and (3).

Thus we can always replace $(\mathbf{p}, \lambda, \vec{x})$ by $\left(\mathbf{p}^{\prime}, \lambda^{\prime}, \vec{x}\right)$ such that $S_{\mathbf{p}, \lambda}^{\vec{x}}=S_{\mathbf{p}^{\prime}, \lambda^{\prime}}^{\vec{x}}$ and the coprime assumptions in both 4.7(4) and 4.8 hold, applied to $\left(\mathbf{p}^{\prime}, \lambda^{\prime}, \vec{x}\right)$. Note also that the above implies that if $\vec{x} \in \mathbb{L}$ is any nontorsion element, then $\operatorname{qgr}^{\mathbb{Z}} S_{\mathbf{p}, \lambda}^{\vec{x}}$ always gives the category of coherent sheaves over a weighted projective line, perhaps with different parameters. 


\subsection{Algebraic Approach to Special CM Modules}

In this subsection we give an algebraic treatment of the special CM $S^{\vec{x}}$-modules, and show how to determine the rank one special CM modules without using geometric arguments. Hence this subsection is independent of $\S 3$, and the techniques developed will be used later to obtain geometric corollaries. Note however that the geometry is required to deduce that there are no higher rank indecomposable special CM modules; this algebraic approach seems only to be able to deal with the rank one specials.

Consider $\mathbb{X}_{\mathbf{p}, \lambda}$ and let $\vec{x} \in \mathbb{L}$ be an element with normal form $\vec{x}=\sum_{i=1}^{n} a_{i} \vec{x}_{i}+a \vec{c}$ with $a \geq 0$. By 4.10 we can assume, by changing parameters if necessary, that $\left(a_{i}, p_{i}\right)=1$ for all $1 \leq i \leq n$. Then, by 4.7 , there is an equivalence

$$
(-)^{\vec{x}}: \mathrm{CM}^{\mathbb{L}} S \rightarrow \mathrm{CM}^{\mathbb{Z}} S^{\vec{x}}
$$

Below we will often use the identification

$$
S_{\vec{y}-\vec{x}} \cong \operatorname{Hom}_{S}^{\mathbb{L}}(S(\vec{x}), S(\vec{y}))
$$

for any $\vec{x}, \vec{y} \in \mathbb{L}$. Recall that the AR translation functor of $S^{\vec{x}}$ is given by

$$
\tau_{S^{\vec{x}}}:=\operatorname{Hom}_{S^{\vec{x}}}\left(-, \omega_{S^{\vec{x}}}\right) \circ \operatorname{Hom}_{S^{\vec{x}}}\left(-, S^{\vec{x}}\right): \mathrm{CM}^{\mathbb{Z}} S^{\vec{x}} \rightarrow \mathrm{CM}^{\mathbb{Z}} S^{\vec{x}},
$$

where $\omega_{S_{\vec{x}}}$ is the $\mathbb{Z}$-graded canonical module of $S^{\vec{x}}[\mathrm{AR} 2, \mathrm{IT}]$.

Proposition 4.11. With the setup as above, the following statements hold.

(1) There is an isomorphism $\omega_{S \vec{x}} \cong S(\vec{\omega})^{\vec{x}}$.

(2) There is a commutative diagram

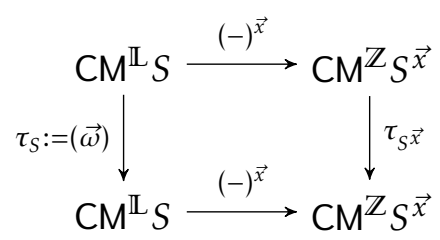

Proof. Again, to ease notation write $R:=S^{\vec{x}}$.

(1) Taking a projective resolution of $\mathbb{k}$ in $\bmod ^{\mathbb{L}} S$, applying $\operatorname{Hom}_{S}(-, S(\vec{\omega}))$ and using 4.6 we see that $\operatorname{Ext}_{R}^{i}\left(\mathbb{k}, S(\vec{\omega})^{\vec{x}}\right)=\operatorname{Ext}_{S}^{i}(\mathbb{k}, S(\vec{\omega}))^{\vec{x}}$. This is $\mathbb{k}$ for $i=2$ and zero for $i \neq 2[\mathrm{BHe}]$. Thus $S(\vec{\omega})^{\vec{x}}$ is the $\mathbb{Z}$-graded canonical module of $R$.

(2) Let $X \in \mathrm{CM}^{\mathbb{L}} S$. Using (1) and 4.6,

$$
\tau_{R}\left(X^{\vec{x}}\right)=\operatorname{Hom}_{R}\left(\operatorname{Hom}_{R}\left(X^{\vec{x}}, S^{\vec{x}}\right), S(\vec{\omega})^{\vec{x}}\right)=\operatorname{Hom}_{S}\left(\operatorname{Hom}_{S}(X, S), S(\vec{\omega})\right)^{\vec{x}}=X(\vec{\omega})^{\vec{x}} .
$$

The following gives an algebraic criterion for certain CM $S^{\vec{x}}$-modules to be special.

Lemma 4.12. For $\vec{y} \in \mathbb{L}$, the $C M S^{\vec{x}}$-module $S(\vec{y})^{\vec{x}}$ is special if and only if

$$
S_{\vec{y}+\vec{\omega}+\ell \vec{x}}=\sum_{m \in \mathbb{Z}} S_{\vec{\omega}+m \vec{x}} \cdot S_{\vec{y}+(\ell-m) \vec{x}}
$$

holds for all $\ell \in \mathbb{Z}$. 
Proof. Set $R:=S^{\vec{x}}$ and as above write $\tau_{R}: \mathrm{CM}^{\mathbb{Z}} R \simeq \mathrm{CM}^{\mathbb{Z}} R$ for the AR-translation. If $\overline{\mathrm{CM}}^{\mathbb{Z}} R$ is the quotient category of $\mathrm{CM}^{\mathbb{Z}} R$ by the ideal generated by $\left\{\omega_{R}(\ell) \mid \ell \in \mathbb{Z}\right\}$, this yields AR duality

$$
D \operatorname{Ext}_{\bmod _{R}}^{1}(X, Y) \simeq \operatorname{Hom}_{\overline{\mathrm{CM}}_{R}}{ }_{R}\left(Y, \tau_{R} X\right)
$$

for any $X, Y \in \mathrm{CM}^{\mathbb{Z}} R[\mathrm{AR} 2, \mathrm{IT}]$. By 4.11(1), $S(\vec{\omega}+\ell \vec{x})^{\vec{x}}=\omega_{R}(\ell)$ holds, and hence there is an induced equivalence

$$
(-)^{\vec{x}}:\left(\mathrm{CM}^{\mathbb{L}} S\right) / I \simeq \overline{\mathrm{CM}}^{\mathbb{Z}} R
$$

for the ideal $I$ of the category $\mathrm{CM}^{\mathbb{L}} S$ generated by add $\{S(\vec{\omega}+\ell \vec{x}) \mid \ell \in \mathbb{Z}\}$. It follows that

$$
\begin{aligned}
D \operatorname{Ext}_{R}^{1}\left(S(\vec{y})^{\vec{x}}, R\right) & =\bigoplus_{\ell \in \mathbb{Z}} D \operatorname{Ext}_{\bmod ^{\mathbb{Z}} R}^{1}\left(S(\vec{y})^{\vec{x}}, R(\ell)\right) \\
& \stackrel{(\stackrel{4 . I)}{\simeq}}{ } \bigoplus_{\ell \in \mathbb{Z}} \operatorname{Hom}_{\overline{C M}_{R}^{Z}}\left(R,\left(\tau_{R}\left(S(\vec{y})^{\vec{x}}\right)\right)(\ell)\right) \\
& \stackrel{(4 . \mathrm{G})(4 . \mathrm{J})}{\simeq} \bigoplus_{\ell \in \mathbb{Z}} \frac{\operatorname{Hom}_{\mathrm{CM}^{\mathbb{I}} S}(S, S(\vec{y}+\vec{w}+\ell \vec{x}))}{I(S, S(\vec{y}+\vec{w}+\ell \vec{x}))} .
\end{aligned}
$$

Thus $S(\vec{y})^{\vec{x}}$ is special if and only if $\operatorname{Hom}_{\mathrm{CM}^{\mathbb{L}} S}(S, S(\vec{y}+\vec{\omega}+\ell \vec{x}))=I(S, S(\vec{y}+\vec{\omega}+\ell \vec{x}))$ holds for all $\ell \in \mathbb{Z}$. Since $\operatorname{Hom}_{\mathrm{CM}^{\mathbb{L}} S}(S, S(\vec{y}+\vec{\omega}+\ell \vec{x}))=S_{\vec{y}+\vec{\omega}+\ell \vec{x}}$ and $I(S, S(\vec{y}+\vec{\omega}+\ell \vec{x}))=\sum_{m \in \mathbb{Z}} S_{\vec{\omega}+m \vec{x}} \cdot S_{\vec{y}+(\ell-m) \vec{x}}$ hold by (4.F), the assertion follows.

We will also require the next result, which is much more elementary, and follows from 2.4.

Lemma 4.13 ([GL1]). Suppose that $\vec{x} \in \mathbb{L}$ has normal form $\vec{x}=\sum_{i=1}^{n} a_{i} \vec{x}_{i}+a \vec{c}$.

(1) If $\vec{y} \in \mathbb{L}_{+}$and $\vec{x}-\vec{y} \in \mathbb{L}_{+}$, write $\vec{y}=\sum_{i=1}^{n} b_{i} \vec{x}_{i}+b \vec{c}$ in normal form. Then for $I:=\left\{1 \leq i \leq n \mid a_{i}<b_{i}\right\}$,

$$
\vec{x} \geq|I| \vec{c} \quad \text { and } \quad S_{\vec{y}} \cdot S_{\vec{x}-\vec{y}}=\left(\prod_{i \in I} x_{i}^{p_{i}}\right) S_{\vec{x}-|I| \vec{c}}
$$

(2) Let $X, Y$ be a basis of $S_{\vec{c}}$. If $\vec{x} \geq i \vec{c} \geq 0$, then

$$
S_{\vec{x}}=X S_{\vec{x}-\vec{c}}+f(X, Y) S_{\vec{x}-i \vec{c}}
$$

for any $f(X, Y) \in S_{i \vec{c}}$ which is not a multiple of $X$.

Before proving the main result 4.15 , we first illustrate a special case.

Example 4.14. Let $\vec{s}_{a}=\sum_{i=1}^{n} \vec{x}_{i}+a \vec{c}$ with $a \geq 0$ and $n+a \geq 2$ (since $a \geq 0$, the last condition is equivalent to $\left.\vec{s}_{a} \notin[0, \vec{c}]\right)$. Then $S(\vec{y})^{\vec{s}_{a}}$ is a special CM $S^{\vec{s}_{a}}$-module for all $\vec{y} \in[0, \vec{c}]$.

Proof. We use 4.12. When $\ell \leq 0$, both sides of (4.H) are zero. When $\ell>0$, since $\vec{\omega}+\vec{s}_{a}=(n-2+a) \vec{c}$ we have

$$
S_{\vec{y}+\vec{\omega}+\ell \vec{S}_{a}}=S_{\vec{y}+(\ell-1) \vec{s}_{a}+(n-2+a) \vec{c}} \stackrel{2.4(3)}{=} S_{(n-2+a) \vec{c}} \cdot S_{\vec{y}+(\ell-1) \vec{s}_{a}}=S_{\vec{\omega}+\vec{s}_{a}} \cdot S_{\vec{y}+(\ell-1) \vec{s}_{a}}
$$

and so (4.H) holds.

The following is the main result in this section. The algebraic method of proof describes all the rank one indecomposable special CM modules directly, and the geometry is only required to verify that there are no further indecomposable special CM modules of higher rank. The algebraic method of proof developed below feeds back into the geometry, and allows us to extract the middle self-intersection number in 4.19. As notation, we write $\mathrm{SCM}^{\mathbb{Z}} S^{\vec{x}}$ for those special CM $S^{\vec{x}}$-modules that are $\mathbb{Z}$-graded. 
Theorem 4.15. Let $\vec{x} \in \mathbb{L}_{+}$with $\vec{x} \notin[0, \vec{c}]$. Write $\vec{x}=\sum_{i=1}^{n} a_{i} \vec{x}_{i}+a \vec{c}$ in normal form, then the following statements hold.

(1) Up to degree shift, the indecomposable objects in $\mathrm{SCM}^{\mathbb{Z}} S^{\vec{x}}$ are precisely those $S\left(u \vec{x}_{j}\right)^{\vec{x}}$ with $1 \leq j \leq n$ and $u \in I\left(p_{j}, p_{j}-a_{j}\right)$.

(2) Forgetting the grading, add $\left\{S\left(u \vec{x}_{j}\right)^{\vec{x}} \mid j \in[1, n], u \in I\left(p_{j}, p_{j}-a_{j}\right)\right\}=\operatorname{SCM} S^{\vec{x}}$.

In particular, $S^{\vec{x}}, S(\vec{c})^{\vec{x}}$ and $S\left(\left(p_{j}-a_{j}\right) \vec{x}_{j}\right)^{\vec{x}}$ for all $j \in[1, n]$ are always special.

Proof. We only prove (1), since the other statements follow immediately. By 4.10(1) we can assume that $\left(a_{i}, p_{i}\right)=1$ for all $1 \leq i \leq n$. Write $R:=S^{\vec{x}}$.

(a) We first claim that, up to degree shift, $\mathbb{Z}$-graded special $C M R$-modules of rank one must have the form $S\left(u \vec{x}_{j}\right)^{\vec{x}}$ for some $1 \leq j \leq n$ and $0 \leq u \leq p_{j}$.

By $2.2 S$ is an $\mathbb{L}$-graded factorial domain, so all rank one objects in $C M^{\mathbb{L}} S$ have the form $S(\vec{y})$ for some $\vec{y} \in \mathbb{L}$. Under the rank preserving equivalence 4.7(1), it follows that all rank one objects in $\mathrm{CM}^{\mathbb{Z}} R$ have the form $S(\vec{y})^{\vec{x}}$ for some $\vec{y} \in \mathbb{L}$. Since we are working up to degree shift, and $\vec{x} \geq 0$, we can assume without loss of generality that $\vec{y} \geq 0$ and $\vec{y} \geq \vec{x}$, by, if necessary, replacing $\vec{y}$ by $\vec{y}-\ell \vec{x}$ for some $\ell \in \mathbb{Z}$.

Hence we can assume that our rank one special CM module has the form $S(\vec{y})^{\vec{x}}$ with $\vec{y} \geq 0$ and $\vec{y} ¥ \vec{x}$. Now assume that $\vec{y}$ can not be written as $u \vec{x}_{j}$ for some $1 \leq j \leq n$ and $0 \leq u \leq p_{j}$. Then there exists $j \neq k$ such that $\vec{y} \geq \vec{x}_{j}+\vec{x}_{k}$. By applying 4.12 for $\ell=0$, it follows that

$$
S_{\vec{y}+\vec{\omega}}=\sum_{m \in \mathbb{Z}} S_{\vec{\omega}+m \vec{x}} \cdot S_{\vec{y}-m \vec{x}}
$$

Now $S_{\vec{y}+\vec{\omega}} \neq 0$ by our assumption $\vec{y} \geq \vec{x}_{j}+\vec{x}_{k}$, hence there exists $m \in \mathbb{Z}$ such that $S_{\vec{\omega}+m \vec{x}} \neq 0$ and $S_{\vec{y}-m \vec{x}} \neq 0$. On one hand, since $\vec{\omega} ¥ 0$, this implies that $m>0$. On the other hand, since $\vec{y} ¥ \vec{x}$, this implies that $m \leq 0$, a contradiction. Thus the rank one special CM modules have the claimed form $S\left(u \vec{x}_{j}\right)^{\vec{x}}$.

(b) Let $1 \leq j \leq n$ and $0 \leq u \leq p_{j}$. We now show that $S\left(u \vec{x}_{j}\right)^{\vec{x}}$ is a special CM $R$-module if and only if $u \in I\left(p_{j}, p_{j}-a_{j}\right)$. By 4.12 , the CM $R$-module $S\left(u \vec{x}_{j}\right)^{\vec{x}}$ is special if and only if

$$
S_{u \vec{x}_{j}+\vec{\omega}+\ell \vec{x}}=\sum_{m \in \mathbb{Z}} S_{\vec{\omega}+m \vec{x}} \cdot S_{u \vec{x}_{j}+(\ell-m) \vec{x}}
$$

holds for all $\ell \in \mathbb{Z}$, or equivalently, for all $\ell>0$ since the left hand side vanishes for $\ell \leq 0$ (in that case we have $u \vec{x}_{j}+\vec{\omega} \leq \vec{c}+\vec{\omega} \nsupseteq 0$ ). Thus in what follows, we fix an arbitrary $\ell>0$.

Clearly equality holds in (4.K) if and only if $\subseteq$ holds. To simplify notation, for $m \in \mathbb{Z}$ write

$$
\begin{aligned}
\vec{x} & :=u \vec{x}_{j}+\vec{\omega}+\ell \vec{x} \\
\vec{y}_{m} & :=\vec{\omega}+m \vec{x} .
\end{aligned}
$$

Then $S_{\vec{y}_{m}} \cdot S_{\vec{x}-\vec{y}_{m}}=S_{\vec{\omega}+m \vec{x}} \cdot S_{u \vec{x}_{j}+(\ell-m) \vec{x}}$. Notice that $\vec{y}_{m}, \vec{x}-\vec{y}_{m} \in \mathbb{L}_{+}$holds if and only if $1 \leq m \leq \ell$. The 'if' part follows easily from $\vec{x} \notin[0, \vec{c}]$, and the 'only if' part follows from $\vec{\omega} ¥ 0$ and $u \vec{x}_{j}-\vec{x} \leq \vec{c}-\vec{x} \nsucceq 0$. Thus (4.K) holds if and only if

$$
S_{\overrightarrow{\mathrm{x}}} \subseteq \sum_{m=1}^{\ell} S_{\vec{y}_{m}} \cdot S_{\overrightarrow{\mathrm{x}}-\vec{y}_{m}}
$$

holds. Note that $\vec{x}$ and $\vec{y}_{m}$ can be written more explicitly as

$$
\left.\begin{array}{l}
\vec{x}=\left(\sum_{i \neq j}\left(\ell a_{i}-1\right) \vec{x}_{i}\right)+\left(u+\ell a_{j}-1\right) \vec{x}_{j}+(n-2+a \ell) \vec{c} \\
\vec{y}_{m}=\sum_{i=1}^{n}\left(m a_{i}-1\right) \vec{x}_{i}+(n-2+a m) \vec{c} .
\end{array}\right\}
$$


Since $\vec{y}_{m}, \vec{x}-\vec{y}_{m} \in \mathbb{L}_{+}$for each $1 \leq m \leq \ell, 4.13(1)$ implies that

$$
S_{\overrightarrow{\mathrm{y}}_{m}} \cdot S_{\overrightarrow{\mathrm{x}}-\overrightarrow{\mathrm{y}}_{m}}=\left(\prod_{i \in I_{m}} x_{i}^{p_{i}}\right) S_{\overrightarrow{\mathrm{x}}-\left|I_{m}\right| \vec{c}}
$$

where $I_{m}$ is the set $I$ in 4.13(1) for $\vec{x}$ and $\vec{y}_{m}$. As before, for an integer $k$, we write $[k]_{p_{i}}$ for the integer $k^{\prime}$ satisfying $0 \leq k^{\prime} \leq p_{i}-1$ and $k-k^{\prime} \in p_{i} \mathbb{Z}$. Simply writing out $\vec{x}$ and $\vec{y}_{m}$ into normal form, from (4.M) we see that

$$
I_{m}=\left\{1 \leq i \leq n \mid\left[u_{i}+\ell a_{i}-1\right]_{p_{i}}<\left[m a_{i}-1\right]_{p_{i}}\right\}
$$

where $u_{i}:=u$ if $i=j$ and $u_{i}:=0$ otherwise.

For the case $m=\ell$, it is clear that $I_{\ell} \subseteq\{j\}$. Hence we see that

$$
S_{\vec{y}_{\ell}} \cdot S_{\vec{x}-\vec{y}_{\ell}} \stackrel{(4 . N)}{=}\left(\prod_{i \in I_{\ell}} x_{i}^{p_{i}}\right) S_{\vec{x}-\left|I_{\ell}\right| \vec{c}} \supseteq x_{j}^{p_{j}} S_{\vec{x}-\vec{c}}
$$

Now we claim that (4.L) holds if and only if $j \notin I_{m}$ for some $1 \leq m \leq \ell$.

$(\Rightarrow)$ Assume that (4.L) holds. If further $j \in I_{m}$ for all $1 \leq m \leq \ell$, then using

$$
S_{\vec{x}} \stackrel{(4 . \mathrm{L})}{=} \sum_{m=1}^{\ell} S_{\vec{y}_{m}} \cdot S_{\vec{x}-\vec{y}_{m}} \stackrel{(4 . \mathrm{N})}{=} \sum_{m=1}^{\ell}\left(\prod_{i \in I_{m}} x_{i}^{p_{i}}\right) S_{\overrightarrow{\mathrm{x}}-\left|I_{m}\right| \vec{c}}
$$

we see that $x_{j}^{p_{j}}$ divides every element in $S_{\vec{x}}$. This gives a contradiction, since we can use the normal form of $\vec{x}$ to obtain elements of $S_{\vec{x}}$ which are not divisible by $x_{j}^{p_{j}}$.

$(\Leftarrow)$ Suppose that $j \notin I_{m}$ for some $1 \leq m \leq \ell$. Since $\vec{x} \geq\left|I_{m}\right| \vec{c} \geq 0$ holds by 4.13(1), we have

$$
S_{\overrightarrow{\mathrm{x}}}=x_{j}^{p_{j}} S_{\overrightarrow{\mathrm{x}}-\vec{c}}+\left(\prod_{i \in I_{m}} x_{i}^{p_{i}}\right) S_{\overrightarrow{\mathrm{x}}-\left|I_{m}\right| \vec{c}}
$$

by choosing $X:=x_{j}^{p_{j}}$ and $f(X, Y):=\prod_{i \in I_{m}} x_{i}^{p_{i}}$ in 4.13(2). Finally, using (4.N) and (4.P) this gives

$$
S_{\vec{x}} \subseteq S_{\vec{y}_{\ell}} \cdot S_{\vec{x}-\vec{y}_{\ell}}+S_{\vec{y}_{m}} \cdot S_{\vec{x}-\vec{y}_{m}},
$$

which clearly implies (4.L).

Consequently, (4.L) holds if and only if $j \notin I_{m}$ for some $1 \leq m \leq \ell$, which by (4.O) holds if and only if $\left[u+\ell a_{j}-1\right]_{p_{j}} \geq\left[m a_{j}-1\right]_{p_{j}}$ for some $1 \leq m \leq \ell$. By 2.21 , this holds if and only if $u \in I\left(p_{j}, p_{j}-a_{j}\right)$, proving claim (b).

(c) We now prove part (1). Combining (a) and (b), it suffices to show that there is no indecomposable object $X$ in $\mathrm{SCM}^{\mathbb{Z}} R$ with rank bigger than one. Otherwise, by $[\mathrm{Y}, 15.2 .1], \widehat{X}$ is an indecomposable object in SCM $\mathcal{R}$ with rank bigger than one, where $\mathcal{K}$ is the completion of $R$. This is a contradiction to 2.9 and 3.15, and so Part (1) follows.

\subsection{The Middle Self-Intersection Number}

In this subsection we use the techniques of the previous subsections to determine the middle self-intersection number in (1.E). This requires the following two elementary but technical lemmas.

Lemma 4.16. Let $\ell_{1}, \ldots, \ell_{m}$ be elements in $S_{\vec{c}}$ such that any two elements are linearly independent. Then $\prod_{j \neq 1} \ell_{j}, \ldots, \prod_{j \neq m} \ell_{j}$ is a basis of $S_{(m-1) \vec{c}}$.

Proof. Assume that the assertion holds for $m-1$. Then $\prod_{j \neq 1} \ell_{j}, \ldots, \prod_{j \neq m-1} \ell_{j}$ gives a basis of $\ell_{m} S_{(m-2) \vec{c}}$. Since $S_{(m-1) \vec{c}}=\ell_{m} S_{(m-2) \vec{c}}+\mathrm{k} \prod_{j \neq m} \ell_{j}$ holds, the assertion also holds for $m$. 
The following lemma is general, and does not require $n>0$.

Lemma 4.17. Let $\vec{x} \in \mathbb{L}_{+}$, and write $\vec{x}=\sum_{i=1}^{n} a_{i} \vec{x}_{i}+a \vec{c}$ in normal form. If $t \geq 2$, then every morphism in $\operatorname{Hom}_{S}^{\mathbb{L}}(S(\vec{c}), S(t \vec{x}))$ factors through $\operatorname{add}\left\{S\left(\vec{x}+\left(p_{i}-a_{i}\right) \vec{x}_{i}\right) \mid 1 \leq i \leq n\right\}$.

Proof. It suffices to show that

$$
S_{t \vec{x}-\vec{c}} \subset \sum_{i=1}^{n} S_{\vec{x}-a_{i} \vec{x}_{i}} \cdot S_{(t-1) \vec{x}-\left(p_{i}-a_{i}\right) \vec{x}_{i}} .
$$

For each $i$ with $1 \leq i \leq n$, take $m_{i} \geq 0$ and $\varepsilon_{i} \in\{0,1\}$ such that

$$
(t-1) a_{i}=\left[(t-1) a_{i}\right]_{p_{i}}+m_{i} p_{i} \text { and } t a_{i}=\left[t a_{i}\right]_{p_{i}}+\left(m_{i}+\varepsilon_{i}\right) p_{i} .
$$

Let $m:=\sum_{i=1}^{n} m_{i}$ and $\varepsilon:=\sum_{i=1}^{n} \varepsilon_{i}$. Then the equality

$$
t \vec{x}-\vec{c}=\sum_{j=1}^{n}\left[t a_{j}\right]_{p_{j}} \vec{x}_{j}+(m+\varepsilon-1+t a) \vec{c}
$$

implies that

$$
S_{t \vec{x}-\vec{c}}=\left(\prod_{j=1}^{n} x_{j}^{\left[t a_{j}\right]_{p_{j}}}\right) S_{(m+\varepsilon-1+t a) \vec{c}}
$$

Similarly the equality

$$
(t-1) \vec{x}-\left(p_{i}-a_{i}\right) \vec{x}_{i}=\left[t a_{i}\right]_{p_{i}} \vec{x}_{i}+\sum_{j \neq i}\left[(t-1) a_{j}\right]_{p_{j}} \vec{x}_{j}+\left(m+\varepsilon_{i}-1+(t-1) a\right) \vec{c}
$$

implies that

$$
S_{(t-1) \vec{x}-\left(p_{i}-a_{i}\right) \vec{x}_{i}}=x_{i}^{\left[t a_{i}\right]_{p_{i}}}\left(\prod_{j \neq i} x_{j}^{\left[(t-1) a_{j}\right]_{p_{j}}}\right) S_{\left(m+\varepsilon_{i}-1+(t-1) a\right) \vec{c}}
$$

Multiplying $S_{\vec{x}-a_{i} \vec{x}_{i}}=\left(\prod_{j \neq i} x_{j}^{a_{j}}\right) S_{a \vec{c}}$ and using $\left[(t-1) a_{j}\right]_{p_{j}}+a_{j}=\left[t a_{j}\right]_{p_{j}}+\varepsilon_{j} p_{j}$ gives

$$
S_{\vec{x}-a_{i} \vec{x}_{i}} \cdot S_{(t-1) \vec{x}-\left(p_{i}-a_{i}\right) \vec{x}_{i}}=\left(\prod_{j=1}^{n} x_{j}^{\left[t a_{j}\right]_{p_{j}}}\right)\left(\prod_{j \neq i} x_{j}^{\varepsilon_{j} p_{j}}\right) S_{a \vec{c}} \cdot S_{\left(m+\varepsilon_{i}-1+(t-1) a\right) \vec{c}} \cdot
$$

Now set $I:=\left\{1 \leq i \leq n \mid \varepsilon_{i}=1\right\}$. Clearly $|I|=\varepsilon$ holds.

First we assume $I \neq \emptyset$. By 4.16 we have $\sum_{i \in I} \mathbb{k}_{j \neq i} x_{j}^{\varepsilon_{j} p_{j}}=S_{(\varepsilon-1) \vec{c}}$ and thus

$$
\begin{aligned}
\sum_{i \in I} S_{\vec{x}-a_{i} \vec{x}_{i}} \cdot S_{(t-1) \vec{x}-\left(p_{i}-a_{i}\right) \vec{x}_{i}} & \stackrel{(4 . \mathrm{R})}{=}\left(\prod_{j=1}^{n} x_{j}^{\left[t a_{j}\right]_{p_{j}}}\right) S_{(\varepsilon-1) \vec{c}} \cdot S_{a \vec{c}} \cdot S_{(m+(t-1) a) \vec{c}} \\
& =\left(\prod_{j=1}^{n} x_{j}^{\left[t a_{j}\right]_{p_{j}}}\right) S_{(m+\varepsilon-1+t a) \vec{c}} \\
& \stackrel{(4 . \mathrm{O})}{=} S_{t \vec{x}-\vec{c},}
\end{aligned}
$$

as desired.

Next we assume $I=\emptyset$. If further $m-1+(t-1) a \geq 0$, then (4.R) is equal to

$$
\left(\prod_{j=1}^{n} x_{j}^{\left[t a_{j}\right]_{p_{j}}}\right) S_{a \vec{c}} \cdot S_{(m-1+(t-1) a) \vec{c}}=\left(\prod_{j=1}^{n} x_{j}^{\left[t a_{j}\right]_{p_{j}}}\right) S_{(m-1+t a) \vec{c}} \stackrel{(4.0)}{=} S_{t \vec{x}-\vec{c}},
$$

as desired, so we can assume that $m-1+(t-1) a<0$. But $a \geq 0$ since $\vec{x} \in \mathbb{L}_{+}$, and $t \geq 2$ by assumption, so necessarily $m=0=a$. Then $m-1+t a<0$ holds, so $S_{t \vec{x}-\vec{c}}=0$ by (4.Q), which implies the assertion. 
The following is the main result of this subsection; the main point is that the manipulations above involving the combinatorics of the weighted projective line give the geometric corollary in 4.19 below.

Theorem 4.18. Let $\vec{x} \in \mathbb{L}_{+}$with $\vec{x} \notin[0, \vec{c}]$, and write $\vec{x}=\sum_{i=1}^{n} a_{i} \vec{x}_{i}+a \vec{c}$ in normal form. Set $R:=S^{\vec{x}}$ and $N:=S(\vec{c})^{\vec{x}}$, and consider their completions $\mathcal{K}$ and $\widehat{N}$. Then in the quiver of the reconstruction algebra of $\mathcal{K}$, the number of arrows from $\widehat{N}$ to $\mathcal{K}$ is a.

Proof. By $4.10(2), S_{\mathbf{p}, \lambda}^{\vec{x}} \cong S_{\mathbf{p}^{\prime}, \lambda^{\prime}}^{\vec{x}}$ as $\mathbb{Z}$-graded algebras, where $\vec{x}:=\sum_{i \in I} a_{i} \vec{x}_{i}+a \vec{c} \in \mathbb{L}^{\prime}$ satisfies the condition in 4.7(4). Note that this change in parameters has not changed the value $a$ on $\vec{c}$, hence in what follows, we can assume that $\mathrm{CM}^{\mathbb{L}} S \simeq \mathrm{CM}^{\mathbb{Z}} R$ holds, via the functor $(-)^{\vec{x}}$.

Let $\mathcal{C}$ be the full subcategory of $\mathrm{CM}^{\mathbb{L}} S$ corresponding to $\mathrm{SCM}^{\mathbb{Z}} R$ via the functor $(-)^{\vec{x}}$. Then the number of arrows from $\widehat{N}$ to $\mathcal{K}$ is equal to the dimension of the $\mathbb{k}$-vector space

$$
\frac{\operatorname{rad}_{\mathrm{SCMk}}(\widehat{N}, \mathfrak{R})}{\operatorname{rad}_{\mathrm{SCM} \mathfrak{R}}^{2}(\widehat{N}, \mathfrak{R})} \cong \prod_{t \in \mathbb{Z}} \frac{\operatorname{Hom}_{R}^{\mathbb{Z}}(N, R(t))}{\operatorname{rad}_{\mathrm{SCM}^{\mathbb{Z}} R}^{2}(N, R(t))} \cong \prod_{t \in \mathbb{Z}} \frac{\operatorname{Hom}_{S}^{\mathbb{L}}(S(\vec{c}), S(t \vec{x}))}{\operatorname{rad}_{\mathcal{C}}^{2}(S(\vec{c}), S(t \vec{x}))} .
$$

By $4.15, \mathcal{C}$ is the additive closure of $S\left(u \vec{x}_{j}+s \vec{x}\right)$, where $s \in \mathbb{Z}, 1 \leq j \leq n$ and $u \in I\left(p_{j}, p_{j}-a_{j}\right)$. We split into three cases.

(1) If $t \leq 0$, then $\operatorname{Hom}_{S}^{\mathbb{L}}(S(\vec{c}), S(t \vec{x}))=0$.

(2) If $t \geq 2$, then since $S\left(\vec{x}+\left(p_{i}-a_{i}\right) \vec{x}_{i}\right)$ belongs to $\mathcal{C}$ by 4.15 , and is not isomorphic to both $S(\vec{c})$ and $S(t \vec{x})$ in $\bmod ^{\mathbb{L}} S$ (since $\left.\vec{x} \notin[0, \vec{c}]\right)$, we have $\operatorname{Hom}_{S}^{\mathbb{L}}(S(\vec{c}), S(t \vec{x}))=\operatorname{rad}_{\mathcal{C}}^{2}(S(\vec{c}), S(t \vec{x}))$ by 4.17 .

(3) Suppose that $t=1$. By definition any morphism in $\operatorname{rad}_{\mathcal{C}}^{2}(S(\vec{c}), S(\vec{x}))$ can be written as a sum of

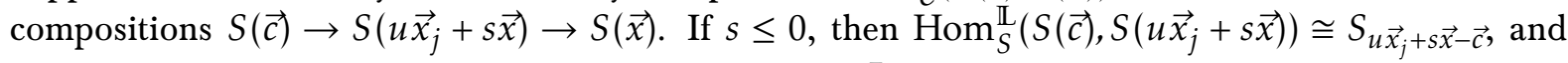
hence $\operatorname{rad}_{\mathcal{C}}\left(S(\vec{c}), S\left(u \vec{x}_{j}+s \vec{x}\right)\right)=0$. If $s \geq 1$, then $\operatorname{Hom}_{S}^{\mathbb{L}}\left(S\left(u \vec{x}_{j}+s \vec{x}\right), S(\vec{x})\right) \cong S_{(1-s) \vec{x}-u \overrightarrow{x_{j}}}$, and hence $\operatorname{rad}_{\mathcal{C}}\left(S\left(u \vec{x}_{j}+s \vec{x}\right), S(\vec{x})\right)=0$. Either way, $\operatorname{rad}_{\mathcal{C}}^{2}(S(\vec{c}), S(t \vec{x}))=0$ in this case.

Combining all cases, the desired number is thus

$$
\sum_{t \in \mathbb{Z}} \operatorname{dim}_{\mathbb{k}}\left(\frac{\operatorname{Hom}_{S}^{\mathbb{L}}(S(\vec{c}), S(t \vec{x}))}{\operatorname{rad}_{\mathcal{C}}^{2}(S(\vec{c}), S(t \vec{x}))}\right)=\operatorname{dim}_{\mathbb{k}} \operatorname{Hom}_{S}^{\mathbb{L}}(S(\vec{c}), S(\vec{x}))=\operatorname{dim}_{\mathbb{k}} S_{\vec{x}-\vec{c}} \stackrel{2.4}{=} a .
$$

This allows us to finally complete the proof of 1.5 from the introduction.

Corollary 4.19. Let $\vec{x} \in \mathbb{L}_{+}$with $\vec{x} \notin[0, \vec{c}]$, and write $\vec{x}=\sum_{i=1}^{n} a_{i} \vec{x}_{i}+a \vec{c}$ in normal form. Then the morphism $\pi: Y^{\vec{x}} \rightarrow \operatorname{Spec} S^{\vec{x}}$ is the minimal resolution, and its dual graph is precisely $(1 . \mathrm{E})$ with $\beta=a+v=a+\#\left\{i \mid a_{i} \neq 0\right\}$.

Proof. We know from 3.17 that $\pi$ is the minimal resolution, and we know from construction of $Y^{\vec{x}}$ that all the self-intersection numbers are determined by the continued fraction expansions (\$2.4), except the middle curve $E_{i}$ corresponding to the special CM module $S(\vec{c})^{\vec{x}}$. The dual graph does not change under completion. By 4.18 the number of arrows in the reconstruction algebra from the middle vertex to the vertex $\circ$ is $a$. Thus the calculation (2.B) combined with 2.12 shows that $a=-E_{i} \cdot Z_{f}=\beta-v$.

\subsection{The Reconstruction Algebra and its qgr}

Using the above subsections, we next describe the quiver of the reconstruction algebra and determine the associated qgr category. Consider the dual graph (1.E), then with the convention that we only draw the 
arms that are non-empty, we see from $3.15,(2 . \mathrm{B})$ and $Z_{K} \cdot E_{i}=E_{i}^{2}+2$ that

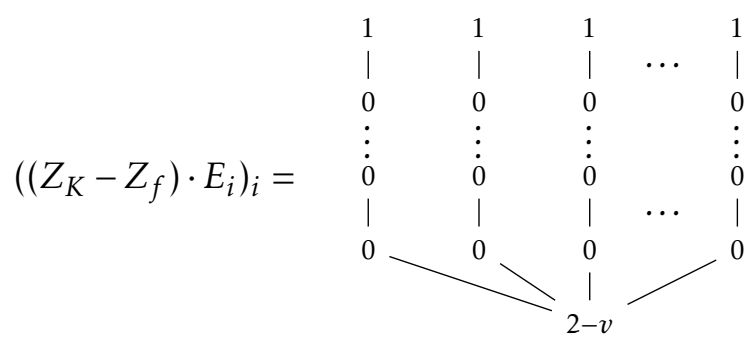

Note that the cases $v=0$ and $v=1$ are degenerate, and are already well understood [W3]. Therefore in the next result, we only consider the case $v \geq 2$.

Inspecting the list of special CM $S^{\vec{x}}$-modules in 3.18, the conditions in 2.11 are satisfied, so we consider the particular choice of reconstruction algebra

$$
\Gamma_{\vec{x}}:=\operatorname{End}_{S \vec{x}}\left(M^{\vec{x}}\right) \quad \text { where } \quad M:=S \oplus\left(\bigoplus_{j \in[1, n], u} S\left(u \vec{x}_{j}\right)\right) \oplus S(\vec{c}),
$$

and $u$ in the middle direct sum ranges over $I\left(p_{j}, p_{j}-a_{j}\right) \backslash\left\{0, p_{j}\right\}$. Since the above $S^{\vec{x}}$-modules are clearly $\mathbb{Z}$-graded, this induces a $\mathbb{Z}$ grading on $\Gamma_{\vec{x}}$.

Corollary 4.20. For $\vec{x} \in \mathbb{L}_{+}$with $\vec{x} \notin[0, \vec{c}]$, write $\vec{x}=\sum_{i=1}^{n} a_{i} \vec{x}_{i}+$ a $\vec{c}$ in normal form. For each $i$ with $a_{i} \neq 0$, as before $m_{i}$ is defined via $\frac{p_{i}}{p_{i}-a_{i}}=\left[\alpha_{i 1}, \ldots, \alpha_{i m_{i}}\right]$, and if $a_{i}=0$ set $m_{i}=0$. Suppose that $v=\#\left\{i \mid a_{i} \neq 0\right\}$ satisfies $v \geq 2$. Then the reconstruction algebra $\Gamma_{\vec{x}}$ can be presented as a quiver with relations, where the relations are homogeneous with respect to the natural grading, and the quiver is the following: we first consider the double quiver of the dual graph (1.E) and add an extending vertex (denoted o) as follows:

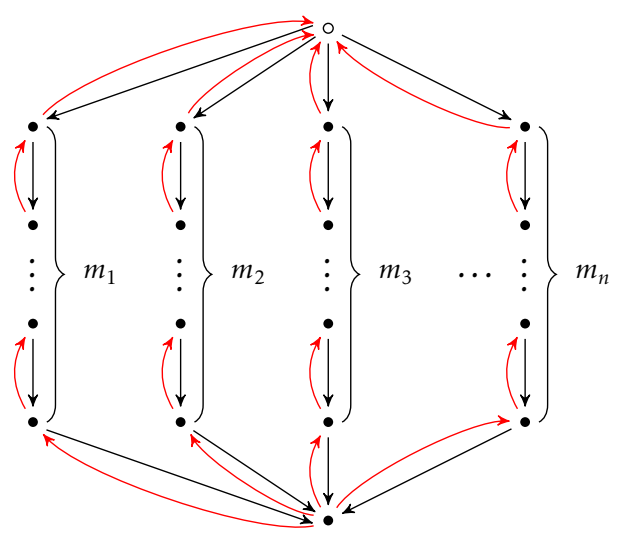

where by convention if $m_{i}=0$ the ith arm does not exist. Further, we add extra arrows subject to the following rules:

(1) If some $\alpha_{i j}>2$, add $\alpha_{i j}-2$ extra arrows from that vertex to the top vertex.

(2) Add further a arrows from the bottom vertex to the top vertex.

Proof. As in [W4, §4], we first work on the completion $\Pi_{i \in \mathbb{Z}} \operatorname{Hom}_{S^{\vec{x}}}^{\mathbb{Z}}\left(M^{\vec{x}}, M^{\vec{x}}(i \vec{x})\right)$ of $\Gamma_{\vec{x}}$, where the result follows by combining 3.19, (2.B), (4.S) and 2.12. The result then follows from the easy fact that if $f: \bigoplus_{i \geq 0} A_{i} \rightarrow$ $\bigoplus_{i \geq 0} B_{i}$ is a morphism of graded rings, then $f$ is an isomorphism if and only $\widehat{f}: \prod_{i \geq 0} A_{i} \rightarrow \prod_{i \geq 0} B_{i}$ is an isomorphism. 
It is possible to describe the relations of $\Gamma_{\vec{x}}$ in this level of generality, but for notational ease we will only do this for the 0-Wahl Veronese in $\S 5$ below. However, in full generality, we do have the following.

Proposition 4.21. Suppose that $\vec{x}=\sum_{i=1}^{n} a_{i} \vec{x}_{i}+a \vec{c} \in \mathbb{L}_{+}$with $\vec{x} \notin[0, \vec{c}]$. Then, with notation as in 4.20, $\left(\Gamma_{\vec{x}}\right)_{0}$, the degree zero part of the reconstruction algebra $\Gamma_{\vec{x}}$, is isomorphic to the canonical algebra $\Lambda_{\mathbf{q}, \mu}$, where $I:=\left\{i \in[1, n] \mid a_{i} \neq 0\right\}, \mathbf{q}:=\left(m_{i}+1\right)_{i \in I}$ and $\mu:=\left(\lambda_{i}\right)_{i \in I}$.

Proof. By 4.10 we can change parameters to assume that the coprime assumption $4.7(4)$ holds. Thus we have $\mathrm{CM}^{\mathbb{Z}} S^{\vec{x}} \simeq \mathrm{CM}^{\mathbb{L}} S$ and hence $\left(\Gamma_{\vec{x}}\right)_{0} \cong \operatorname{End}_{S}^{\mathbb{L}}(M)$. But by [GL1] it is well known that there is a ring isomorphism $\operatorname{End}_{S}^{\mathbb{L}}\left(\bigoplus_{\vec{y} \in[0, \vec{c}]} S(\vec{y})\right) \cong \Lambda_{\mathbf{p}, \lambda}$. Thus $\left(\Gamma_{\vec{x}}\right)_{0}=e \Lambda_{\mathbf{p}, \lambda} e$ for an idempotent $e$ corresponding to a subset of $[0, \vec{c}]$ containing 0 and $\vec{c}$. Clearly $e \Lambda_{\mathbf{p}, \lambda} e \cong \Lambda_{\mathbf{q}, \mu}$ for $\mathbf{q}$ and $\mu$ in the statement.

When $\vec{x} \in \mathbb{L}_{+}$with $\vec{x} \notin[0, \vec{c}]$, we will next show in 4.22 that qgr ${ }^{\mathbb{Z}} S^{\vec{x}} \simeq \mathrm{qgr}^{\mathbb{Z}} \Gamma_{\vec{x}}$, since after combining with 4.10 this then allows us to realise any weighted projective line as $\operatorname{qgr}^{\mathbb{Z}} \Gamma_{\vec{x}}$. Since $\Gamma_{\vec{x}}$ is an $\mathbb{N}$-graded ring, this allows us to interpret any weighted projective line as a 'noncommutative projective scheme' over 'Spec $\left(\Gamma_{\vec{x}}\right)_{0}$ ', and thus by 4.21 a noncommutative projective scheme over the canonical algebra.

Proposition 4.22. For $\vec{x} \in \mathbb{L}_{+}$with $\vec{x} \notin[0, \vec{c}]$, there is an equivalence

$$
\operatorname{qgr}^{\mathbb{Z}} S^{\vec{x}} \simeq \operatorname{qgr}^{\mathbb{Z}} \Gamma_{\vec{x}}
$$

where $\Gamma_{\vec{x}}$ is a $\mathbb{Z}$-graded $\mathbb{k}$-algebra such that $\left(\Gamma_{\vec{x}}\right)_{i}$ is $\Lambda_{\mathbf{q}, \mu}$ for $i=0$ and zero for $i<0$.

Proof. Set $R:=S^{\vec{x}}$. Let $A:=\Gamma_{\vec{x}}=\operatorname{End}_{R}\left(M^{\vec{x}}\right)$ and let $e \in A$ be the idempotent corresponding to the summand $R$ of $M^{\vec{x}}$. Clearly $B:=e A e \cong R$. Note that $\operatorname{dim}_{\mathbb{k}}(A /\langle e\rangle)<\infty$ since by $2.3(3) R$ is normal, so $\operatorname{add} M_{\mathfrak{p}}^{\vec{x}}=\operatorname{add} R_{\mathfrak{p}}$ for any non-maximal prime ideal $\mathfrak{p}$ of $R$, and hence $(A /\langle e\rangle)_{\mathfrak{p}}=0$. The first statement then follows from 4.1 , and the second statement by 4.21 .

\section{The 0-Wahl Veronese}

Throughout this section we work with an arbitrary $\mathbb{X}_{\mathbf{p}, \lambda}$ with $n \geq 3$, and consider the 0 -Wahl Veronese subring of $S=S_{\mathbf{p}, \lambda}$ from the introduction, namely $S^{\vec{s}}$, where $\vec{s}=\sum_{i=1}^{n} \vec{x}_{i}$. It is not too hard, but more notationally complicated, to extend to cover the case $\vec{s}_{a}=\vec{s}+a \vec{c}$, but we shall not do this here. We investigate the more general $S^{\vec{s}_{a}}$ for Dynkin type in $\S 6$.

\subsection{Presenting the 0 -Wahl Veronese}

The aim of this subsection is to give a presentation of the 0 -Wahl Veronese subring $S^{\vec{s}}$ of $S$ by constructing an isomorphism $S^{\vec{s}} \cong R_{\mathbf{p}, \lambda}$. We define elements of $S^{\vec{s}}$ as follows:

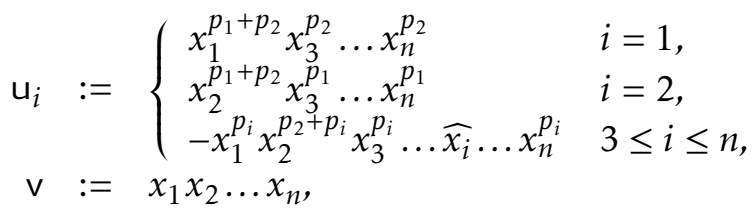

where we write $\widehat{x_{i}}$ to mean 'omit $x_{i}$ '. Then with respect to the $\mathbb{Z}$-grading $S^{\vec{s}}=\bigoplus_{i \in \mathbb{Z}} S_{i \vec{s}}$, the element $\vee$ is homogeneous of degree one, and $\mathrm{u}_{i}$ is homogeneous of degree $p_{2}$ if $i=1, p_{1}$ if $i=2$ and $p_{i}$ if $3 \leq i \leq n$.

To construct an isomorphism between $R_{\mathbf{p}, \lambda}$ and $S^{\vec{s}}$, we first construct a morphism of graded algebras.

Lemma 5.1. The morphism $\mathbb{k}\left[u_{1}, \ldots, u_{n}, v\right] \rightarrow S^{\vec{s}}$ of graded algebras given by $u_{i} \mapsto \mathrm{u}_{i}$ for $1 \leq i \leq n$ and $v \mapsto \mathrm{v}$ induces a morphism $R_{\mathbf{p}, \lambda} \rightarrow S^{\vec{s}}$ of graded algebras. 
Proof. It suffices to show that all $2 \times 2$ minors of the following matrix have determinant zero.

$$
\left(\begin{array}{ccccc}
\mathrm{u}_{2} & \mathrm{u}_{3} & \cdots & \mathrm{u}_{n} & \mathrm{v}^{p_{2}} \\
\mathrm{v}^{p_{1}} & \lambda_{3} \mathrm{u}_{3}+\mathrm{v}^{p_{3}} & \ldots & \lambda_{n} \mathrm{u}_{n}+\mathrm{v}^{p_{n}} & \mathrm{u}_{1}
\end{array}\right)
$$

Since $S^{\vec{s}}$ is a domain, it suffices to show that all $2 \times 2$ minors containing the last column have determinant zero. The outer $2 \times 2$ minor has determinant

$$
\mathrm{u}_{1} \mathrm{u}_{2}-\mathrm{v}^{p_{1}+p_{2}}=\left(x_{1} \ldots x_{n}\right)^{p_{1}+p_{2}}-\left(x_{1} \ldots x_{n}\right)^{p_{1}+p_{2}}=0 .
$$

Further for any $i \geq 3$, using the relation $x_{1}^{p_{1}}=\lambda_{i} x_{2}^{p_{2}}-x_{i}^{p_{i}}$ it follows that

$$
\begin{aligned}
\mathrm{u}_{1} \mathrm{u}_{i} & =-x_{1}^{p_{1}+p_{2}+p_{i}} x_{2}^{p_{2}+p_{i}} x_{3}^{p_{2}+p_{i}} \ldots x_{i}^{p_{2}} \ldots x_{n}^{p_{2}+p_{i}} \\
& =-\left(\lambda_{i} x_{2}^{p_{2}}-x_{i}^{p_{i}}\right) x_{1}^{p_{2}+p_{i}} x_{2}^{p_{2}+p_{i}} x_{3}^{p_{2}+p_{i}} \ldots x_{i}^{p_{2}} \ldots x_{n}^{p_{2}+p_{i}} \\
& =x_{1}^{p_{2}} x_{2}^{p_{2}} \ldots x_{n}^{p_{2}}\left(-\lambda_{i} x_{1}^{p_{i}} x_{2}^{p_{2}+p_{i}} x_{3}^{p_{i}} \ldots \widehat{x_{i}} \ldots x_{n}^{p_{i}}+x_{1}^{p_{i}} x_{2}^{p_{i}} \ldots x_{n}^{p_{i}}\right) \\
& =v^{p_{2}}\left(\lambda_{i} \mathrm{u}_{i}+v^{p_{i}}\right) .
\end{aligned}
$$

Thus the $2 \times 2$ minor consisting of $i$ th column and the last one has determinant zero.

The following calculation is elementary.

Proposition 5.2. (1) The $\mathbb{k}$-algebra $S^{\vec{s}}$ is generated by $\vee$ and $\mathrm{u}_{i}$ with $1 \leq i \leq n$.

(2) The $\mathbb{k}$-vector space $S^{\vec{s}} / \mathrm{v} S^{\vec{s}}$ is generated by $\mathrm{u}_{i}^{\ell}$ with $1 \leq i \leq n$ and $\ell \geq 0$.

Proof. It is enough to prove (2). Let $V$ be the subspace of $S^{\vec{s}} / v S^{\vec{s}}$ generated by $u_{i}^{\ell}$ with $1 \leq i \leq n$ and $\ell \geq 0$. Take any monomial $X:=x_{1}^{a_{1}} \ldots x_{n}^{a_{n}}$ in $S_{N \vec{s}}$ with $N>0$, then

$$
a_{1} \vec{x}_{1}+\ldots+a_{n} \vec{x}_{n}=N \vec{x}_{1}+\ldots+N \vec{x}_{n} .
$$

For each $1 \leq i \leq n$, there exists $\ell_{i} \in \mathbb{Z}$ such that $a_{i}=N+\ell_{i} p_{i}$. Then $\sum_{i=1}^{n} \ell_{i}=0$ holds.

(i) We first show that $X$ belongs to $V$ in the situation when there exists $i$ with $1 \leq i \leq n$ satisfying $\ell_{i} \leq 0$, $\ell_{j} \geq 0$ and $\ell_{k}=0$ for all $k \neq i, j$, where $j$ is defined by $j:=2$ if $i \neq 2$ and $j:=1$ if $i=2$.

If $a_{i} \neq 0$, then by the assumptions, all $a_{k} \geq 1$ for $1 \leq k \leq n$, and hence $X$ belongs to $v S^{\vec{s}}$. Thus we can assume that $a_{i}=0$. Then $N=-\ell_{i} p_{i}$ and $a_{j}=N+\ell_{j} p_{j}=-\ell_{i}\left(p_{i}+p_{j}\right)$ hold, and further

$$
X=x_{j}^{a_{j}} \prod_{k \neq i, j} x_{k}^{N}=\left(x_{j}^{p_{i}+p_{j}} \prod_{k \neq i, j} x_{k}^{p_{i}}\right)^{-\ell_{i}}= \pm \mathrm{u}_{i^{\prime}}^{-\ell_{i}},
$$

where $i^{\prime}:=2$ if $i=1, i^{\prime}:=1$ if $i=2$ and $i^{\prime}=i$ if $i \geq 3$. Thus the assertion follows.

(ii) We consider the general case. Using induction on $\ell(X):=\sum_{1 \leq i \leq n, \ell_{i}>0} \ell_{i}$, we show that $X$ belongs to $V$.

Assume $\ell(X)=0$. Then $X=\mathrm{v}^{N}$ holds, and hence $X$ belongs to $V$.

Assume that there exist $1 \leq i \neq j \leq n$ such that $\ell_{i}<0$ and $\ell_{j}<0$. Take $1 \leq k \leq n$ such that $\ell_{k}>0$. Since $\left\{x_{i}^{p_{i}}, x_{j}^{p_{j}}\right\}$ is a $\mathbb{k}$-basis of $S_{\vec{c}}$, there is a relation $x_{k}^{p_{k}}=\lambda^{\prime} x_{i}^{p_{i}}+\lambda^{\prime \prime} x_{j}^{p_{j}}$ with $\lambda^{\prime}, \lambda^{\prime \prime} \in \mathbb{k}$, and we have $X=\lambda^{\prime} X^{\prime}+\lambda^{\prime \prime} X^{\prime \prime}$ for some monomials $X^{\prime}, X^{\prime \prime}$ satisfying $\ell\left(X^{\prime}\right)<\ell(X)$ and $\ell\left(X^{\prime \prime}\right)<\ell(X)$. Since $X^{\prime}$ and $X^{\prime \prime}$ belong to $V$, so does $X$.

In the rest, assume that there exists a unique $1 \leq i \leq n$ satisfying $\ell_{i}<0$. Define $j$ by $j:=2$ if $i \neq 2$ and $j:=1$ if $i=2$. Using the relation $x_{k}^{p_{k}}=\lambda_{k}^{\prime} x_{i}^{p_{i}}+\lambda_{k}^{\prime \prime} x_{j}^{p_{j}}$ with $\lambda_{k}^{\prime}, \lambda_{k}^{\prime \prime} \in \mathbb{k}$, we have

$$
X=x_{i}^{a_{i}} x_{j}^{a_{j}} \prod_{k \neq i, j} x_{k}^{N+\ell_{k} p_{k}}=x_{i}^{a_{i}} x_{j}^{a_{j}} \prod_{k \neq i, j} x_{k}^{N}\left(\lambda_{k}^{\prime} x_{i}^{p_{i}}+\lambda_{k}^{\prime \prime} x_{j}^{p_{j}}\right)^{\ell_{k}} .
$$

This is a linear combination of monomials $Y=x_{i}^{b_{i}} x_{j}^{b_{j}} \prod_{k \neq i, j} x_{k}^{N}$ which satisfies the condition in (i). Thus $X$ belongs to $V$. 
The above leads to the following, which is the main result of this subsection.

Theorem 5.3. There is a graded ring isomorphism $R_{\mathrm{p}, \lambda} \cong S^{\vec{s}}$ given by $u_{i} \mapsto \mathrm{u}_{i}$ for $1 \leq i \leq n$ and $v \mapsto \mathrm{v}$.

Proof. Combining 5.1 and 5.2, there is a surjective graded ring homomorphism $\vartheta: R_{\mathbf{p}, \lambda} \rightarrow S^{\vec{s}}$. But now $R_{\mathbf{p}, \lambda}$, being a rational surface singularity, is automatically a domain. Since $S^{\vec{s}}$ is two-dimensional, $\vartheta$ must be an isomorphism.

\subsection{Special CM $S^{\vec{s}}$-Modules and the Reconstruction Algebra}

The benefit of our Veronese construction of $R_{\mathrm{p}, \lambda}$ is that it also produces the special CM modules, and we now describe them explicitly as 2 -generated ideals. We first do this in the notation of $S$, then translate into the coordinates $\mathrm{u}_{1}, \ldots, \mathrm{u}_{n}, \mathrm{v}$.

Proposition 5.4. The following are, up to degree shift, precisely the indecomposable non-free objects in $\mathrm{SCM}^{\mathbb{Z}} S^{\vec{s}}$. Moreover, they have the following generators and degrees:

\begin{tabular}{ccc}
\hline Module & Generators & Degree of generators \\
\hline$S\left(q \vec{x}_{1}\right)^{\vec{s}}$ & $x_{2}^{p_{2}}\left(x_{2} x_{3} \ldots x_{n}\right)^{p_{1}-q}$ and $x_{1}^{q}$ & $p_{1}-q$ and 0 \\
$S\left(q \vec{x}_{2}\right)^{\vec{s}}$ & $x_{1}^{p_{1}}\left(x_{1} x_{3} \ldots x_{n}\right)^{p_{2}-q}$ and $x_{2}^{q}$ & $p_{2}-q$ and 0 \\
$S\left(q \vec{x}_{i}\right)^{\vec{s}}$ & $x_{2}^{p_{2}}\left(x_{1} \ldots \widehat{x}_{i} \ldots x_{n}\right)^{p_{i}-q}$ and $x_{i}^{q}$ & $p_{i}-q$ and 0 \\
$S(\vec{c})^{\vec{s}}$ & $x_{1}^{p_{1}}$ and $x_{2}^{p_{2}}$ & 0 and 0 \\
\hline
\end{tabular}

where in row one $q \in\left[1, p_{1}-1\right]$, in row two $q \in\left[1, p_{2}-1\right]$, and in row three $i \in[3, n], q \in\left[1, p_{i}-1\right]$.

Proof. The first statement is 4.15(1). We only prove the assertions for $S\left(q \vec{x}_{1}\right)^{\vec{s}}$ since all other cases are similar. Let $M$ be the submodule of $S\left(q \vec{x}_{1}\right)^{\vec{s}}$ generated by $g_{1}:=x_{1}^{q}$ and $g_{2}:=x_{2}^{p_{1}+p_{2}-q} x_{3}^{p_{1}-q} \ldots x_{n}^{p_{1}-q}$. To prove $M=S\left(q \vec{x}_{1}\right)^{\vec{s}}$, it suffices to show that any monomial $X=x_{1}^{a_{1}} \ldots x_{n}^{a_{n}} \in S\left(q \vec{x}_{1}\right)^{\vec{s}}$ of degree $N \geq 0$ has either $g_{1}$ or $g_{2}$ as a factor. Since

$$
a_{1} \vec{x}_{1}+\ldots+a_{n} \vec{x}_{n}=(N+q) \vec{x}_{1}+N \vec{x}_{2}+\ldots+N \vec{x}_{n}
$$

holds, there exists $\ell_{i} \in \mathbb{Z}$ for each $1 \leq i \leq n$ such that $a_{1}=N+q+\ell_{1} p_{1}$ and $a_{i}=N+\ell_{i} p_{i}$ for $i \geq 2$. Then $\sum_{i=1}^{n} \ell_{i}=0$ holds.

(i) If $a_{1} \geq q$, then $X$ belongs to $M$ since $X$ has $g_{1}=x_{1}^{q}$ as a factor.

(ii) We show that $X$ belongs to $M$ if $\ell_{3}=\ldots=\ell_{n}=0$. By (i), we can assume that $a_{1}<q$ and hence $\ell_{1}<0$. Then $N=a_{1}-q-\ell_{1} p_{1} \geq p_{1}-q$ holds. Since $\ell_{2}=-\ell_{1}>0$, we have $a_{2}=N+\ell_{2} p_{2} \geq p_{1}+p_{2}-q$, which implies that $X=x_{1}^{a_{1}} x_{2}^{a_{2}} x_{3}^{N} \ldots x_{n}^{N}$ has $g_{2}=x_{2}^{p_{1}+p_{2}-q} x_{3}^{p_{1}-q} \ldots x_{n}^{p_{1}-q}$ as a factor.

(iii) We show that $X$ belongs to $M$ if all $\ell_{3}, \ldots, \ell_{n}$ are non-positive. By (i), we can assume that $a_{1}<q$ and hence $\ell_{1}<0$. Using $\ell_{2}=-\sum_{i \neq 2} \ell_{i}$ and the relation $x_{2}^{p_{2}}=\lambda_{i}^{\prime} x_{1}^{p_{1}}+\lambda_{i}^{\prime \prime} x_{i}^{p_{i}}$, it follows that

$$
X=x_{1}^{a_{1}} x_{2}^{N-\sum_{i \neq 2} \ell_{i} p_{2}} x_{3}^{a_{3}} \ldots x_{n}^{a_{n}}=x_{1}^{a_{1}} x_{2}^{N-\ell_{1} p_{2}} \prod_{i \geq 3} x_{i}^{a_{i}}\left(\lambda_{i}^{\prime} x_{1}^{p_{1}}+\lambda_{i}^{\prime \prime} x_{i}^{p_{i}}\right)^{-\ell_{i}}
$$

Since $a_{1}+p_{1}>q$, this is a linear combination of monomials which have $g_{1}=x_{1}^{q}$ as a factor and of a monomial $x_{1}^{a_{1}} x_{2}^{N-\ell_{1} p_{2}} \prod_{i \geq 3} x_{i}^{a_{i}-\ell_{i} p_{i}}=x_{1}^{a_{1}} x_{2}^{N-\ell_{1} p_{2}} x_{3}^{N} \ldots x_{n}^{N}$ satisfying (ii). Thus $X$ belongs to $M$.

(iv) We show that $X$ belongs to $M$ in general. Let $\ell_{i}^{+}=\max \left\{\ell_{i}, 0\right\}$ and $\ell_{i}^{-}=\min \left\{\ell_{i}, 0\right\}$, then $\ell_{i}=\ell_{i}^{+}+\ell_{i}^{-}$. Further, using the relation $x_{i}^{p_{i}}=-x_{1}^{p_{1}}+\lambda_{i} x_{2}^{p_{2}}$,

$$
X=x_{1}^{a_{1}} x_{2}^{a_{2}} \prod_{i \geq 3} x_{i}^{N+\ell_{i} p_{i}}=-x_{1}^{a_{1}} x_{2}^{a_{2}} \prod_{i \geq 3} x_{i}^{N+\ell_{i}^{-} p_{i}}\left(-x_{1}^{p_{1}}+\lambda_{i} x_{2}^{p_{2}}\right)^{\ell_{i}^{+}} .
$$

This is a linear combination of monomials satisfying (iii), so $X$ belongs to $M$. 
Using 5.2 we now translate the modules in 5.4 into ideals.

Proposition 5.5. With notation in 5.3, up to degree shift, the non-free indecomposable objects in $\mathrm{SCM}^{\mathbb{Z}} S^{\vec{s}}$ are precisely the following ideals of $S^{\vec{s}}$, and furthermore across the bijection in 2.9 they correspond to the dual graph of the minimal resolution of $\mathrm{Spec} S^{\vec{s}}(1 . \mathrm{F})$ in the following way:

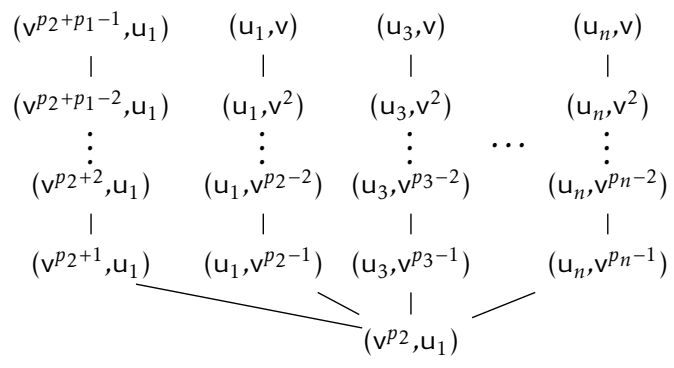

Proof. We first claim that $S\left(\vec{x}_{1}\right)^{\vec{s}} \cong\left(\mathrm{v}^{p_{1}+p_{2}-1}, \mathrm{u}_{1}\right)$. Indeed, since $S$ is an $\mathbb{L}$-domain by 2.2 , multiplication by any homogeneous element $S \rightarrow S$ is injective. Thus, multiplying by $x_{2} \ldots x_{n}$, we see that $S\left(\vec{x}_{1}\right)^{\vec{s}}$ is isomorphic to the $S^{\vec{s}}$-submodule of $S$ generated by $x_{2}^{p_{1}+p_{2}} x_{3}^{p_{1}} \ldots x_{n}^{p_{1}}$ and $x_{1} \ldots x_{n}$, that is generated by $\mathrm{u}_{2}$ and $v$. But then

$$
\mathrm{u}_{1}\left(\mathrm{u}_{2}, \mathrm{v}\right)=\left(\mathrm{u}_{1} \mathrm{u}_{2}, \mathrm{u}_{1} \mathrm{v}\right) \stackrel{5.3}{\cong}\left(\mathrm{v}^{p_{1}+p_{2}}, \mathrm{u}_{1} \mathrm{v}\right)=\left(\mathrm{v}^{p_{1}+p_{2}-1}, \mathrm{u}_{1}\right) \mathrm{v},
$$

which shows that $S\left(\vec{x}_{1}\right)^{\vec{s}} \cong\left(\mathrm{v}^{p_{1}+p_{2}-1}, \mathrm{u}_{1}\right)$. The other cases are similar. The statement regarding the bijection is a special case of 3.19 .

Proposition 5.6. The reconstruction algebra $\Gamma_{\vec{s}}$ is given by the following quiver, where the arrows correspond to the following morphisms.

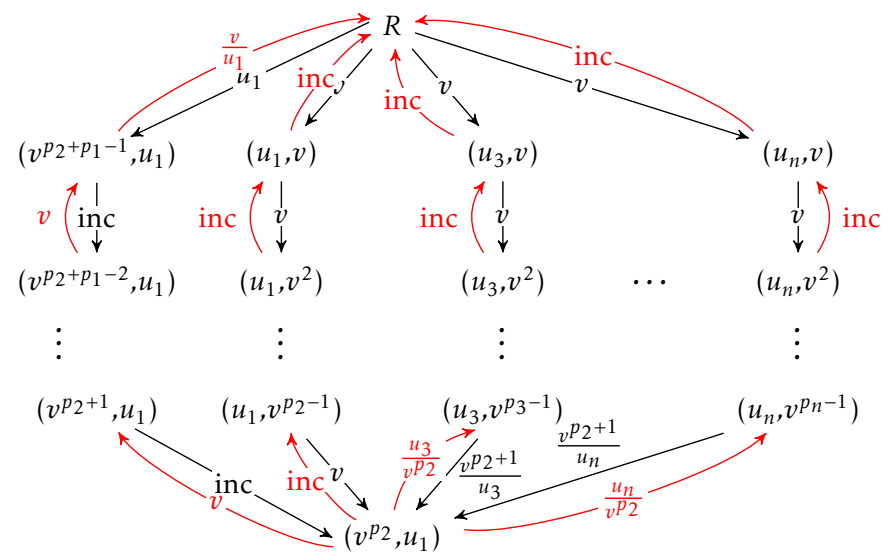

Proof. Under the isomorphisms in 5.3 and 5.5, the morphisms induced by the canonical algebra become

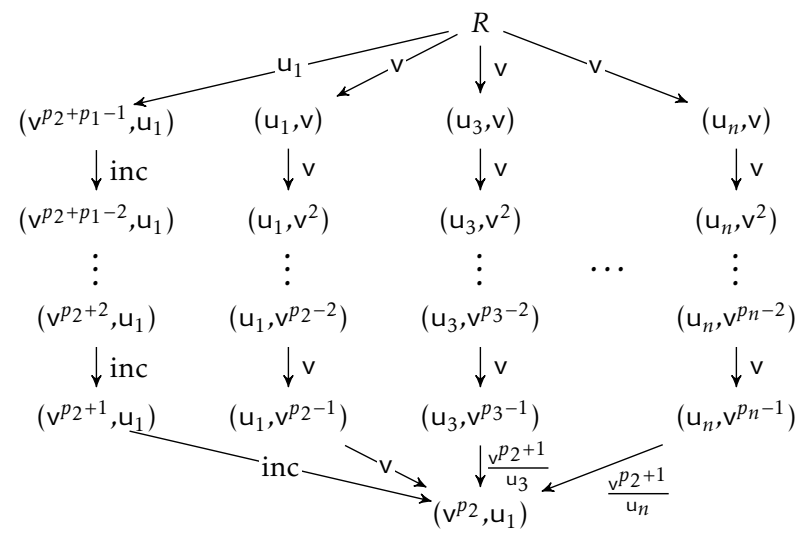


From here, exactly as in the proof of 4.20 , we can work on the completion. We know the quiver of the reconstruction algebra from (4.T), and we know that for every special CM module $X$, we must be able to hit the generators of $X$ by composing arrows starting at the vertex $R$ and ending at the vertex corresponding to $X$, without producing any cycles. Since the arrows in (5.B) are already forced to be arrows in the reconstruction algebra, it remains to choose a basis for the remaining red arrows. For example, the generator $v^{p_{2}+1}$ in $\left(v^{p_{2}+1}, u_{1}\right)$ must come from a composition of arrows $R$ to $\left(v^{p_{2}}, u_{1}\right)$, followed by the bottom left arrow. Since we can see $v^{p_{2}}$ as a composition of maps from $R$ to $\left(v^{p_{2}}, u_{1}\right)$, this forces the bottom left red arrow to be $v$. The remaining arrows are similar.

Theorem 5.7. The reconstruction algebra $\Gamma_{\vec{s}}$ is isomorphic to the path algebra of the double of the quiver $Q_{\mathrm{p}}$, denoted $\bar{Q}_{\mathrm{p}}$, subject to relations given by

(1) The canonical algebra relations on the black arrows

(2) At every vertex, all 2-cycles that exist at that vertex are equal.

Proof. This is very similar to [W4, 4.11]. Set $Q:=\bar{Q}_{\mathbf{p}, \lambda}$ (as in (4.T)), and denote the set of relations in the statement by $\mathcal{S}^{\prime}$. Exactly as in the proof of 4.20 , we can work in the completed case (where we can use [BIRS, 3.4]) and we prove that the completion of reconstruction algebra is given as the completion of $\mathbb{k} Q$ (denoted $\mathbb{k} \widehat{Q})$ modulo the closure of the ideal $\left\langle\mathcal{S}^{\prime}\right\rangle$ (denoted $\left.\overline{\left\langle\mathcal{S}^{\prime}\right\rangle}\right)$. The non-completed version of the theorem then follows.

By 5.6 there is a natural surjection $\gamma: \mathbb{k} \widehat{Q} \rightarrow \hat{\Gamma}$ with $\mathcal{S}^{\prime} \subseteq I:=\operatorname{Ker} \gamma$. Denote the radical of $\mathbb{k} \widehat{Q}$ by $J$ and further let $V$ denote the set of vertices of $Q$. Below we show that the elements of $\mathcal{S}^{\prime}$ are linearly independent in $I /(I J+J I)$, hence we may extend $\mathcal{S}^{\prime}$ to a basis $\mathcal{S}$ of $I /(I J+J I)$. Since $\mathcal{S}$ is a basis, by [BIRS, 3.4(a)] $I=\overline{\langle\mathcal{S}\rangle}$, so it remains to show that $\mathcal{S}=\mathcal{S}^{\prime}$. But by [BIRS, 3.4(b)]

$$
\#\left(e_{a} \mathbb{k} \widehat{Q} e_{b}\right) \cap \mathcal{S}=\operatorname{dim} \operatorname{Ext}_{\hat{\Gamma}}^{2}\left(S_{a}, S_{b}\right)
$$

for all $a, b \in V$, where $S_{a}$ is the simple module corresponding to vertex $a$. From 4.20 (i.e. [W2]), this is equal to some number given by intersection theory. Simply inspecting our set $\mathcal{S}^{\prime}$ and comparing to the numbers in 4.20 , we see that

$$
\#\left(e_{a} \mathbb{k} \widehat{Q} e_{b}\right) \cap \mathcal{S}=\#\left(e_{a} \mathbb{k} \widehat{Q} e_{b}\right) \cap \mathcal{S}^{\prime}
$$

for all $a, b \in V$, proving that the number of elements in $\mathcal{S}$ and $\mathcal{S}^{\prime}$ are the same. Hence $\mathcal{S}^{\prime}=\mathcal{S}$ and so $I=\overline{\left\langle\mathcal{S}^{\prime}\right\rangle}$, as required.

Thus it suffices to show that the elements of $\mathcal{S}^{\prime}$ are linearly independent in $I /(I J+J I)$. This is identical to the proof of [W4, 4.12], so we omit the details.

Whilst thinking of the special CM modules as ideals makes everything much more explicit, doing this forgets the grading. Indeed, the reconstruction algebra $\Gamma_{\vec{s}}$ has a natural grading induced from the Veronese construction.

Proposition 5.8. The reconstruction algebra $\Gamma_{\vec{s}}$ is generated in degree one over its degree zero piece, which is the canonical algebra $\Lambda_{\mathrm{p}, \lambda}$.

Proof. It is clear that all the black arrows in the quiver in 5.6 have degree zero. It is easy to see that any red arrow in the reverse direction to an arrow labelled $x_{i}$ has label $x_{1} \ldots \widehat{x}_{i} \ldots x_{n}$, and it is easy to check that these all have degree one, using 5.4. Hence the degree zero piece is the canonical algebra, and as an algebra $\Gamma_{\vec{s}}$ is generated in degree one over its degree zero piece.

Note that for $a>0$, the reconstruction algebra $\Gamma_{\overrightarrow{s_{a}}}$ is not always generated in degree one over its degree zero piece. 


\section{Domestic Case}

In this section we investigate the domestic case, that is when the dual graph is an ADE Dynkin diagram, and relate Ringel's work on the representation theory of the canonical algebra to the classification of the special CM modules for quotient singularities in [IW]. This will explain the motivating coincidence from the introduction. Since this involves AR theory, typically in this section rings will be complete.

Throughout this section we consider $\mathbb{X}=\mathbb{X}_{\mathbf{p}, \lambda}$ and $S=S_{\mathbf{p}, \lambda}$ with $n=3$ and one of the triples $\left(p_{1}, p_{2}, p_{3}\right)=(2,3,3),(2,3,4)$ or $(2,3,5)$. For $m \geq 3$, we consider $\vec{s}_{m-3}=\vec{s}+(m-3) \vec{c}$ with $\vec{s}=\sum_{i=1}^{3} \vec{x}_{i}$, the $(m-3)$-Wahl Veronese subring $R=S^{\vec{s}_{m-3}}$, and its completion $\mathcal{K}$. Recall that here $\vec{\omega}=\vec{c}-\vec{s}$, since $n=3$.

Proposition 6.1. In the above setting, $\operatorname{Spec} R$ has the following dual graph:

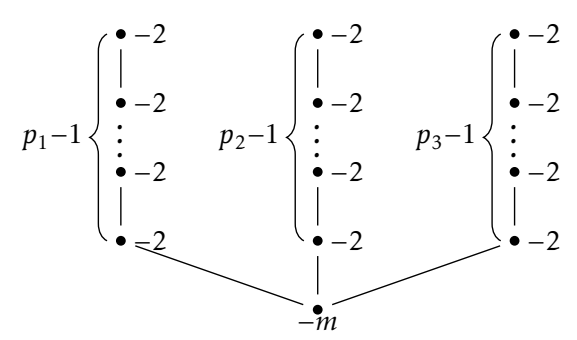

Moreover $\mathfrak{R}$ is isomorphic to a quotient singularity $\mathbb{k}[[x, y]]^{G}$ in the following list:

\begin{tabular}{|c|c|c|}
\hline$\left(p_{1}, p_{2}, p_{3}\right)$ & Dual Graph & $G$ \\
\hline$(2,3,3)$ & $-2-\dot{-}-\dot{i}-\dot{m}-\dot{-}-\dot{-}$ & $\mathbb{T}_{6(m-2)+1}$ \\
\hline$(2,3,4)$ & $-\dot{2}-\dot{-}-\dot{i}-\dot{m}-\dot{-}-\mathbf{-}-\dot{2}$ & $\mathbb{O}_{12(m-2)+1}$ \\
\hline$(2,3,5)$ & $-i-i-i$ & $\mathbb{I}_{30(m-2)+1}$ \\
\hline
\end{tabular}

For the precise definition of the above subgroups of $\mathrm{GL}(2, \mathrm{k})$ we refer the reader to [IW].

Proof. By 4.19, the dual graph of $R$ is known to be (1.F). On the other hand, the quotient singularity $\mathbb{k}[[x, y]]^{G}$ has the same dual graph $[\mathrm{R} 1, \S 3]$. Since the dual graphs (1.F) for ADE triples are known to be taut [B3, Korollar 2.12], the result follows.

Let us finally explain why Ringel's picture (1.A) in the introduction is the same as the ones found in [IW] and [W2, §4]. For example, in the family of groups $\mathbb{O}_{12(m-2)+1}$ with $m \geq 3$ in 6.1, by [AR] the AR quiver of $\mathfrak{R} \cong \mathbb{k}[[x, y]]^{\mathbb{O}_{12(m-2)+1}}$ is

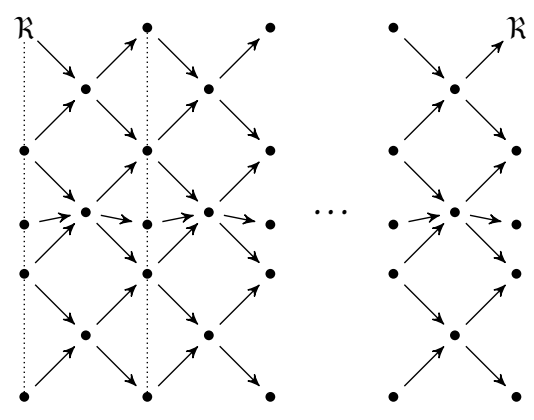


where there are precisely $12(m-2)+1$ repetitions of the original $\tilde{E}_{7}$ shown in dotted lines. The left and right hand sides of the picture are identified, and there is no twist in this AR quiver. Thus as $m$ increases (and the group $\mathbb{O}_{12(m-2)+1}$ changes), the AR quiver becomes longer.

Regardless of $m \geq 3$, by [IW, 8.2] the special CM $\mathcal{K}$-modules always have the following position in the AR quiver:

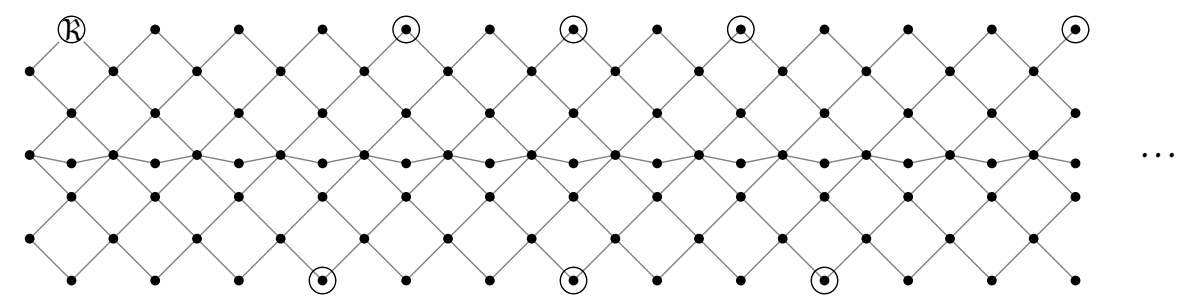

In particular, comparing this to (1.A), we observe the following coincidences.

(1) The AR quiver of CM $\mathcal{R}$ is the quotient of the AR quiver of vect $\mathbb{X}$ by $\tau^{12(m-2)+1}=((12(m-2)+1) \vec{\omega})$.

(2) The canonical tilting bundle $\mathcal{E}$ on $\mathbb{X}$ is given by the circled vertices in (1.A), and so under the identification in (1), this gives the additive generator of SCM $R$.

The same coincidence can also be observed for type $\mathbb{T}$ and II by replacing 12 by 6 and 30 respectively. To give a theoretical explanation to these observations, we need the following preparation.

Lemma 6.2. Define $h$ as follows

\begin{tabular}{cc}
\hline Type & $h$ \\
\hline $\mathbb{T}$ & 6 \\
$\mathbb{O}$ & 12 \\
$\mathbb{I}$ & 30 \\
\hline
\end{tabular}

Then $(h+1) \vec{\omega}=-\vec{s}$ and $(h(m-2)+1) \vec{\omega}=-\vec{s}_{m-3}$.

Proof. If $\left(p_{1}, p_{2}, p_{3}\right)=(2,3,3)$, then $6 \vec{\omega}=(6-3-2-2) \vec{c}=-\vec{c}$ and so $7 \vec{\omega}=-\vec{s}$. Similarly, in the case $\left(p_{1}, p_{2}, p_{3}\right)=(2,3,4)$ then $12 \vec{\omega}=(12-6-4-3) \vec{c}=-\vec{c}$, thus $13 \vec{\omega}=-\vec{s}$. Lastly, if $\left(p_{1}, p_{2}, p_{3}\right)=(2,3,5)$ then $30 \vec{\omega}=(30-15-10-6) \vec{c}=-\vec{c}$, hence $31 \vec{\omega}=-\vec{s}$.

Therefore $(h(m-2)+1) \vec{\omega}=-(m-2) \vec{s}-(m-3) \vec{\omega}=-\vec{s}-(m-3) \vec{c}=-\vec{s}_{m-3}$.

Let $\mathcal{C}$ be an additive category with an action by a cyclic group $G=\langle g\rangle \cong \mathbb{Z}$. Assume that, for any $X, Y \in \mathcal{C}, \operatorname{Hom}_{\mathcal{C}}\left(X, g^{i} Y\right)=0$ holds for $i \gg 0$. The complete orbit category $\mathcal{C} / G$ has the same object as $\mathcal{C}$ and the morphism sets are given by

$$
\operatorname{Hom}_{\mathcal{C} / G}(X, Y):=\prod_{i \in \mathbb{Z}} \operatorname{Hom}_{\mathcal{C}}\left(X, g^{i} Y\right)
$$

for $X, Y \in \mathcal{C}$, where the composition is defined in the obvious way.

Theorem 6.3. Let $R$ be the $(m-3)$-Wahl Veronese subring associated with $\left(p_{1}, p_{2}, p_{3}\right)=(2,3,3),(2,3,4)$ or $(2,3,5)$ and $m \geq 3$, and $\mathcal{R}$ its completion. Let $G \leq \mathbb{L}$ be the infinite cyclic group generated by the element $-\vec{s}_{m-3}=(h(m-2)+1) \vec{\omega}$. Then

(1) There are equivalences vect $\mathrm{X} \simeq \mathrm{CM}^{\mathbb{Z}} R$ and

$$
F:(\operatorname{vect} X) / G \stackrel{\sim}{\rightarrow} \mathrm{CMR} .
$$

(2) For the canonical tilting bundle $\mathcal{E}$ on $\mathrm{X}$, we have $\mathrm{SCM} \mathcal{R}=\operatorname{add} F \mathcal{E}$. 
Proof. Since $(h(m-2)+1) \vec{\omega}=-\vec{s}_{m-3}$ is a non-zero element in $-\mathbb{L}_{+}$, for any $X, Y \in \operatorname{vect} X$, necessarily $\operatorname{Hom}_{X}(X, Y(i(h(m-2)+1) \vec{\omega}))=0$ holds for $i \gg 0$. Therefore the complete orbit category $(\operatorname{vect} X) / G$ is well-defined.

(1) There are equivalences vect $\mathrm{X} \simeq \mathrm{CM}^{\mathbb{L}} S \simeq \mathrm{CM}^{\mathbb{Z}} R$, where the first equivalence is standard [GL1], and the second is 4.7. Furthermore, the following diagram commutes.

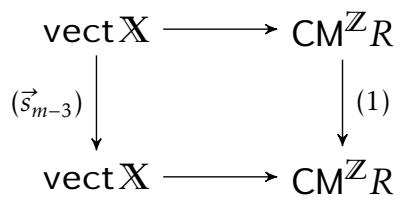

Since $\mathfrak{K}$ has only finitely many indecomposable $\mathrm{CM}$ modules (see e.g. [Y, 15.14]), there is an equivalence $\left(\mathrm{CM}^{\mathbb{Z}} R\right) / \mathbb{Z} \simeq \mathrm{CM} R$. Therefore $(\operatorname{vect} \mathbb{X}) / G \simeq\left(\mathrm{CM}^{\mathbb{Z}} R\right) / \mathbb{Z} \simeq \mathrm{CM}$ k.

(2) This follows by the equivalences in (1), the definition of $\mathcal{E}$, and 3.18 .

As one final observation, recall that for a canonical algebra $\Lambda=\Lambda_{\mathrm{p}, \lambda}$, the preprojective algebra of $\Lambda$ is defined by

$$
\Pi:=\bigoplus_{i \geq 0} \Pi_{i}, \quad \Pi_{i}:=\operatorname{Hom}_{\mathrm{D}^{\mathrm{b}}(\bmod \Lambda)}\left(\Lambda, \tau^{-i} \Lambda\right),
$$

where $\tau$ is the Auslander-Reiten translation in the derived category $\mathrm{D}^{\mathrm{b}}(\bmod \Lambda)$. Moreover, for a positive integer $t$, we denote the $t$-th Veronese subring of $\Pi$ by

$$
\Pi^{(t)}:=\bigoplus_{i \geq 0} \Pi_{t i}
$$

As notation we write $\Gamma_{m}$ for the reconstruction algebra of $R$ above, which corresponds to one of the types $\mathbb{T}, \mathbb{O}$ or $\mathbb{I}$ in 6.1 .

The following is an analogue of 4.21, but also describes the other graded pieces.

Proposition 6.4. There is an isomorphism of $\mathbb{Z}$-graded algebras

$$
\Pi^{(h(m-2)+1)} \cong \Gamma_{m}
$$

Proof. By 6.2 we know that $(h(m-2)+1) \vec{\omega}=-\vec{s}_{m-3}$. Setting $M=\bigoplus_{\vec{y} \in[0, \vec{c}]} S(\vec{y})$, then $\Pi_{i}^{(h(m-2)+1)}$ for $i \geq 0$ is given by

$$
\begin{aligned}
\operatorname{Hom}_{D^{\mathrm{b}}(\bmod \Lambda)}\left(\Lambda, \tau^{-(h(m-2)+1) i} \Lambda\right) & \cong \operatorname{Hom}_{S}^{\mathbb{L}}(M, M(-i(h(m-2)+1) \vec{\omega})) \\
& \cong \operatorname{Hom}_{S}^{\mathbb{L}}\left(M, M\left(i \vec{s}_{m-3}\right)\right) \\
& \cong\left(\Gamma_{m}\right)_{i} .
\end{aligned}
$$

Thus all the graded pieces match. It is easy to see that the isomorphisms are natural, and so give an isomorphism of graded rings.

Remark 6.5. By 6.4, it follows that in fact on the abelian level

$$
\operatorname{qgr}^{\mathbb{Z}} \Gamma_{m} \simeq \operatorname{qgr}^{\mathbb{Z}} \Pi^{(h(m-2)+1)}
$$

and so, combining 4.7 and 4.22 ,

$$
\operatorname{coh} \mathbb{X} \simeq \operatorname{qgr}^{\mathbb{Z}} \Pi^{(h(m-2)+1)}
$$

for any $m \geq 3$. This is a stronger version of results of [GL1] and Minamoto [M], which combine to say that for the weighted projective lines of non-tubular type there are derived equivalences

$$
\mathrm{D}^{\mathrm{b}}(\operatorname{coh} \boldsymbol{X}) \simeq \mathrm{D}^{\mathrm{b}}(\bmod \Lambda) \simeq \mathrm{D}^{\mathrm{b}}\left(\mathrm{qgr}^{\mathbb{Z}} \Pi\right) \text {. }
$$




\section{References}

[AU] T. Abdelgadir and K. Ueda, Weighted projective lines as fine moduli spaces of quiver representations, Comm. Algebra 43 (2015), no. 2, 636-649. MR-3274027

[A] M. Artin, On isolated rational singularities of surfaces, Amer. J. Math. 88 (1966), 129-136. MR-0199191

[AR] M. Auslander and I. Reiten, McKay quivers and extended Dynkin diagrams, Trans. Amer. Math. Soc. 293 (1986), no. 1, 293-301. MR-0814923

[AR2] M. Auslander and I. Reiten, Almost split sequences for $\mathbb{Z}$-graded rings, Singularities, representation of algebras, and vector bundles (Lambrecht, 1985) Lecture Notes in Math., 1273, Springer, Berlin, 1987, pp. 232-243. MR-915178

[B1] A. A. Beilinson, The derived category of coherent sheaves on $\mathbb{P}^{n}$, Selected translations, Selecta Math. Soviet. 3 (1983/84), no. 3, 233-237. MR-0863137

[BLS] D. Bergh, V. Lunts and O. Schnr̈er, Geometricity for derived categories of algebraic stacks, Selecta Math. (N.S.) 22 (2016), no. 4, 2535-2568. MR-3573964

[B2] T. Bridgeland, t-structures on some local Calabi-Yau varieties, J. Algebra 289 (2005), no. 2, 453-483. MR-2142382

[B3] E. Brieskorn, Rationale singularitäten komplexer flächen, Invent. Math. 4 (1968), 336-358. MR0222084

[BS] M. P. Brodmann and R. Y. Sharp, Local cohomology: an algebraic introduction with geometric applications, Cambridge Studies in Advanced Mathematics, vol. 60, Cambridge University Press, Cambridge, 1998. MR-1613627

[BHe] W. Bruns and J. Herzog, Cohen-Macaulay rings, Cambridge Studies in Advanced Mathematics, vol. 39, Cambridge University Press, Cambridge, 1993. MR-1251956

[B4] N. Bourbaki, Commutative algebra. Chapters 1-7, Elements of Mathematics (Berlin), Springer-Verlag, Berlin, 1998. MR-1727221

[BH] R. Buchweitz and L. Hille, Higher Representation-Infinite Algebras from Geometry. In: Representation theory of quivers and finite dimensional algebras (abstracts from the workshop held February 16-22, 2014, organized by William Crawley-Boevey, Osamu Iyama, Bernhard Keller and Henning Krause), pp. 466-470, Oberwolfach Rep. 11 (2014), no. 1, 453-529. MR-3379303

[BIRS] A. Buan, O. Iyama, I. Reiten, and D. Smith, Mutation of cluster-tilting objects and potentials, Amer. J. Math. 133 (2011), no. 4, 835-887. MR-2823864

[CCZ] J. Chen, X.-W. Chen, and Z. Zhou, Monadicity theorem and weighted projective lines of tubular type, Int. Math. Res. Not. IMRN 2015, no. 24, 13324-13359. MR-3436148

[EGA] J. Dieudonné and A. Grothendieck, Éléments de géométrie algébrique, Inst. Hautes Études Sci. Publ. Math. 4 (1960), 8, 11 (1961), 17 (1963), 20, 24 (1964), 28 (1966), 32 (1967).

[D] I. Dolgachev, On algebraic properties of algebras of automorphic forms. In: Modular functions in analysis and number theory, pp. 20-29, Lecture Notes Math. Statist., vol. 5, Univ. Pittsburgh, Pittsburgh, PA, 1983. MR-0732959

[DW] W. Donovan and M. Wemyss, Contractions and deformations, Amer. J. Math. 141 (2019), no. 3, 563592. MR-3956515 
[GL1] W. Geigle and H. Lenzing, A class of weighted projective curves arising in representation theory of finitedimensional algebras. In: Singularities, representation of algebras, and vector bundles (Lambrecht, 1985), pp. 265-297, Lecture Notes in Math., vol. 1273, Springer, Berlin, 1987. MR-0915180

[GL2] W. Geigle and H. Lenzing, Perpendicular categories with applications to representations and sheaves, J. Algebra 144 (1991), no. 2, 273-343. MR-1140607

[H1] R. Hartshorne, Algebraic geometry, Graduate Texts in Mathematics, vol. 52. Springer-Verlag, New York-Heidelberg, 1977. MR-0463157

[H2] M. Hashimoto, Equivariant class group. III. Almost principal fiber bundles. Preprint 2015. arXiv: 1503.02133

[HIMO] M. Herschend, O. Iyama, H. Minamoto, and S. Oppermann, Representation theory of Geigle-Lenzing complete intersections, to appear Mem. Amer. Math. Soc.

[H3] J. Herzog, Ringe mit nur endlich vielen Isomorphieklassen von maximalen, unzerlegbaren CohenMacaulay-Moduln, Math. Ann. 233 (1978), no. 1, 21-34. MR-0463155

[I] Y. Ito, Special McKay correspondence. In: Geometry of toric varieties, pp. 213-225, Sémin. Congr., vol. 6, Soc. Math. France, Paris, 2002. MR-2075612

[IL] O. Iyama and B. Lerner, Tilting bundles on orders on $\mathbb{P}^{d}$, Israel J. Math. 211 (2016), no. 1, 147-169. MR-3474959

[IT] O. Iyama and R. Takahashi, Tilting and cluster tilting for quotient singularities, Math. Ann. 356 (2013), no. 3, 1065-1105. MR-3063907

[IW] O. Iyama and M. Wemyss, The classification of special Cohen Macaulay modules, Math. Z. 265 (2010), no. 1, 41-83. MR-2606949

[IW2] O. Iyama and M. Wemyss, Maximal modifications and Auslander-Reiten duality for non-isolated singularities, Invent. Math. 197 (2014), no. 3, 521-586. MR-3251829

[KM] J. Kollár and S. Mori, Birational geometry of algebraic varieties, Cambridge Tracts in Mathematics, vol. 134, Cambridge Univ. Press, Cambridge, 1998. MR-1658959

[L] H. Laufer, Taut two-dimensional singularities, Math. Ann. 205 (1973), 131-164. MR-0333238

[M] H. Minamoto, Ampleness of two-sided tilting complexes, Int. Math. Res. Not. IMRN 2012, no. 1, 67-101. MR-2874928

[OW] P. Orlik and P. Wagreich, Isolated singularities of algebraic surfaces with $\mathbb{C}^{*}$ action, Ann. of Math. (2) 93 (1971), 205-228. MR-0284435

[P] H. Pinkham, Normal surface singularities with $\mathbb{C}^{*}$ action, Math. Ann. 227 (1977), no. 2, 183-193. MR-0432636

[R1] O. Riemenschneider, Die Invarianten der endlicher Untergruppen von $G L(2, \mathbb{C})$, Math. Z. 153 (1977), no. 1, 37-50. MR-0447230

[R2] C. M. Ringel, Tame algebras and integral quadratic forms, Lecture Notes in Mathematics, vol. 1099, Springer-Verlag, Berlin, 1984. MR-0774589

[R3] D. Rydh, If the direct image of $f$ preserves coherent sheaves on noetherian schemes, how to show $f$ is proper? URL (version: 2014-10-14): https://mathoverflow.net/q/182902. 
[VdB] M. van den Bergh, Three-dimensional flops and noncommutative rings, Duke Math. J. 122 (2004), no. 3, 423-455. MR-2057015

[VdB2] M. van den Bergh, Non-commutative crepant resolutions. In: The legacy of Niels Henrik Abel, pp. 749-770, Springer, Berlin, 2004. MR-2077594

[W1] J. M. Wahl, Equations defining rational singularities, Ann. Sci. École Norm. Sup. (4) 10 (1977), no. 2, 231-263. MR-0444655

[W2] M. Wemyss, The GL(2, C) McKay correspondence, Math. Ann. 350 (2011), no. 3, 631-659. MR2805639

[W3] M. Wemyss, Reconstruction algebras of type A, Trans. Amer. Math. Soc. 363 (2011), no. 6, 3101-3132. MR-2775800

[W4] M. Wemyss, Reconstruction algebras of type D (I), J. Algebra 356 (2012), 158-194. MR-2891127

[W5] J. Wunram, Reflexive modules on cyclic quotient surface singularities. In: Singularities, representation of algebras, and vector bundles (Lambrecht, 1985), pp. 221-231, Lecture Notes in Mathematics, vol. 1273, Springer, Berlin, 1987. MR-0915177

[W6] J. Wunram, Reflexive modules on quotient surface singularities, Math. Ann. 279 (1988), no. 4, 583-598. MR-0926422

[Y] Y. Yoshino, Cohen-Macaulay modules over Cohen-Macaulay rings, London Mathematical Society Lecture Note Series, vol. 146, Cambridge University Press, Cambridge, 1990. MR-1079937

[ZS] O. Zariski and P. Samuel, Commutative algebra. Vol. II. Graduate Texts in Mathematics, vol. 29, Springer-Verlag, New York-Heidelberg, 1975. MR-0389876 